\title{
ON A PHASE-FIELD MODEL FOR ELECTROWETTING AND OTHER ELECTROKINETIC PHENOMENA*
}

\author{
M. A. FONTELOS ${ }^{\dagger}$, G. GRÜN ${ }^{\ddagger}$, AND S. JÖRRES ${ }^{\ddagger}$
}

\begin{abstract}
In three space dimensions, we present existence results for weak solutions to a novel two-phase model for various electrokinetic phenomena, including in particular dynamic electrowetting with electrolytes. The model is thermodynamically consistent. It combines Navier-Stokes- and Cahn-Hilliard-type phase-field equations with Nernst-Planck equations for ion density-evolution and with an elliptic transmission problem for the electrostatic potential. As physical energy estimates guarantee only boundedness of ion densities in the $L \log L$-Orlicz class uniformly with respect to time, an iteration method is proposed to establish higher regularity and integrability results of these quantities. In an appendix, the derivation of the model is sketched.
\end{abstract}

Key words. electrowetting, electrolytes, phase-field model, Navier-Stokes system, CahnHilliard equation, Nernst-Planck equation, free boundary problem in PDE, elliptic transmission problem, regularity iteration, $L \log L$-Orlicz class, existence of weak solutions in three dimensions

AMS subject classifications. 35D05, 35D10, 35R35, 76M30

DOI. $10.1137 / 090779668$

1. Introduction. Electrokinetic phenomena are mechanical effects caused by the motion of ions in liquids. These ions appear, e.g., as dissociated salts or acids and are called electrolytes. In response to applied electric fields, they begin to move and induce fluid flow. This is one reason for the importance of electrokinetic phenomena in microfluidics where they are used to manipulate tiny amounts of liquid.

In this paper, we study a two-phase diffuse interface model for electrokinetic phenomena which particularly takes into account wall effects ("electrowetting"). Given an electrolyte solution which may partially wet a solid wall, which is surrounded by an ambient fluid, and which is subjected to an external electric field, we investigate existence results for the corresponding system of evolution equations. It reads as follows:

$$
\begin{gathered}
\mathbf{v}_{t}+(\mathbf{v} \cdot \nabla) \mathbf{v}-\nabla \cdot(\eta(\phi) \mathbf{T}(\mathbf{v}))+\nabla p=\mu \nabla \phi-\left(\rho_{1}-\rho_{2}\right) \nabla V \text { in } \Omega_{T}, \\
\nabla \cdot \mathbf{v}=0 \text { in } \Omega_{T}, \\
\frac{D}{D t} \rho_{0}-\nabla \cdot\left(K(\phi) \nabla \rho_{0}\right)=R\left(\rho_{1}, \rho_{2}, \rho_{0}, \phi\right) \text { in } \Omega_{T}, \\
\frac{D}{D t} \rho_{i}-\nabla \cdot\left(K(\phi) \rho_{i} \nabla\left((-1)^{i+1} V+\log \rho_{i}\right)\right) \\
=-R\left(\rho_{1}, \rho_{2}, \rho_{0}, \phi\right) \text { in } \Omega_{T} \text { for } i \in\{1,2\},
\end{gathered}
$$

\footnotetext{
* Received by the editors December 10, 2009; accepted for publication (in revised form) November 17, 2010; published electronically February 22, 2011. This work was supported by DAAD, Ministerio de Educación y Ciencia through the program Acciones Integradas Hispano Alemanas, project D/06/12788, and by Dr. Hertha und Helmut Schmauser-Stiftung.

http://www.siam.org/journals/sima/43-1/77966.html

${ }^{\dagger}$ Instituto de Ciencias Matemáticas (ICMAT, CSIC-UAM-UCM-UC3M), C/ Serrano 123, 28006 Madrid, Spain (marco.fontelos@uam.es).

${ }_{\ddagger}^{\ddagger}$ Department Mathematik, Friedrich-Alexander-Universität Erlangen-Nürnberg, Martensstr. 3, 91058 Erlangen, Germany (gruen@am.uni-erlangen.de, joerres@am.uni-erlangen.de).
} 


$$
\begin{aligned}
& \frac{D}{D t} \phi-\nabla \cdot(M(\phi) \nabla \mu)=0 \text { in } \Omega_{T}, \\
& -\nabla \cdot(\bar{\varepsilon}[\phi] \nabla V)=\left(\rho_{1}-\rho_{2}\right) \chi_{\Omega} \text { in } \Omega^{*} \text { for all } t \in(0, T)
\end{aligned}
$$

with the boundary conditions

$$
\begin{gathered}
\mathbf{v}=\mathbf{0}, \\
K(\phi) \nabla \rho_{0} \cdot \mathbf{n}=0, \\
K(\phi) \rho_{i} \nabla\left((-1)^{i+1} V+\log \rho_{i}\right) \cdot \mathbf{n}=0 \text { for } i \in\{1,2\}, \\
\nabla \mu \cdot \mathbf{n}=0, \\
|\nabla \phi|^{s-2} \nabla \phi \cdot \mathbf{n}=-\gamma_{f s}^{\prime}(\phi)-\alpha \phi_{t}
\end{gathered}
$$

on $\partial \Omega \times(0, T)$,

$$
V=\bar{V} \text { on } \partial \Omega^{*} \times(0, T)
$$

and initial conditions to be specified later on.

Here, $\mathbf{v}$ denotes the velocity field, and $\phi$ is the phase field $(\phi \equiv 1$ and $\phi \equiv-1$ indicate the pure phases of the electrolyte solution and the ambient fluid, respectively). Besides the solvent, we assume the electrolyte solution to consist of three species. The density of the first species - a neutral one - will be denoted by $\rho_{0}$. Molecules of this species may dissociate in anions and cations which set up the other two species and which will be denoted by $\rho_{1}$ and $\rho_{2}$, respectively. $R\left(\rho_{1}, \rho_{2}, \rho_{0}, \phi\right)$ is an appropriate recombination/dissociation term. The variables $p, \mu$, and $V$ denote pressure, chemical potential, and electrostatic potential, respectively, and $\chi_{\Omega}$ stands for the characteristic function of $\Omega$. The velocity strain tensor $\mathbf{T}(\mathbf{v})$ and the chemical potential $\mu$ are given by

$$
\mathbf{T}(\mathbf{v})=\frac{1}{2}\left(\nabla \mathbf{v}+(\nabla \mathbf{v})^{t}\right), \quad \mu=-\Delta_{s} \phi+W^{\prime}(\phi)-\frac{1}{2} \varepsilon^{\prime}(\phi)|\nabla V|^{2},
$$

where $-\Delta_{s} \phi=-\nabla \cdot\left(|\nabla \phi|^{s-2} \nabla \phi\right)$ denotes the $s$-Laplacian. Already in [2], the $s$ Laplacian has been used in the context of phase-field models for binary flows with nonmatched densities to describe interfacial energies.

The fluid domain $\Omega$ is compactly contained in a larger domain $\Omega^{*}$ to model the solid wall, too. In typical electrowetting-on-dielectric experiments, electrodes are located at $\partial \Omega^{*}$. They enter the model via Dirichlet boundary conditions $\bar{V}$ for the electrostatic potential $V$ on $\partial \Omega^{*}$.

This model can be derived by Onsager's variational principle [19] and energy considerations in the spirit of [11] and [21]. For a brief sketch and also for some remarks on the sharp-interface case, see Appendix A.

In recent years, different aspects of electrowetting have been studied in the mathematical and physical literature, certainly motivated by the immense importance of electrowetting and more general electrokinetic phenomena as tools in microfluidic applications (see, e.g., $[4,5,6,7,11,14,16,17,18,20,22,23,28,29,31,32,33]$ and the references therein).

Phase-field models for dynamic electrowetting in arbitrary space dimensions were first suggested and analyzed in [11]. There, the focus was on conductive liquids. 
Hence, the "charge density" $\rho$ was allowed to change sign. Mathematically, existence was established in two space dimensions - in three dimensions only under the additional assumption that the dielectric parameters of the liquids are identical.

In the present paper, we liberate ourselves from such limitations. Further analytical difficulties arise due to nonnegativity requirements on $\rho_{0}, \rho_{1}, \rho_{2}$ and in particular due to the fact that the physical energy estimate provides estimates for $\rho_{1}, \rho_{2}$ only in the $L \log L$-Orlicz class (see [3, Chap. 8]), but no regularity of gradients of any power of $\rho_{1}, \rho_{2}$.

Hence, the core of this paper consists of an iteration method which entails $L^{\infty}\left(L^{2}\right) \cap$ $L^{2}\left(H^{1}\right)$-regularity locally in $\Omega$ of positive powers of $\rho_{0}, \rho_{1}, \rho_{2}$. Due to the particular structure of the system - an elliptic transmission problem for $V$ and generalized noflux boundary conditions for $\rho_{1}, \rho_{2}$ on $\partial \Omega$-analogues of these results globally on $\Omega$ seem to be out of reach at the moment.

In the literature, nonlinear drift-diffusion systems in electrophoretic and semiconductor modeling were studied in [12]. There, a coupling with neither hydrodynamic nor phase-field equations is considered, and therefore the present paper requires different techniques. Results on analysis and numerics of a model coupling the NavierStokes equations with a Nernst-Planck system and a potential equation were presented in $[24,25]$. For analytical results on coupled Navier-Stokes-Cahn-Hilliard systems we refer, e.g., to [1, 2], and for numerical analysis we refer to [13]. Note that [2] seems to be so far the only analytical result for models taking liquids with different densities into account (see also [15] for a derivation).

The outline of the paper is as follows: In the remainder of this section, assumptions on the data are specified and the main existence theorem is stated. Its proof requires various regularizations and approximations. In section 2, we prove an existence result for a viscoelastic auxiliary model with an appropriate mobility regularization in the ion equations which we call a VEMR model. An important feature of the corresponding solutions is the approximation-parameter-dependent $L^{\infty}\left(L^{2}\right) \cap L^{2}\left(H^{1}\right)$ regularity. This property is essential to establish in section 3 localized higher regularity results for $\rho_{0}, \rho_{1}, \rho_{2}$ in these VEMR models, which are independent of all the approximation parameters and which are hence the key to proving the main existence theorem, Theorem 1.1. In the appendices, we explain the physical background of the paper (Appendix A), and we study a basic auxiliary problem needed in the existence proof for the aforementioned VEMR model (Appendix B). In particular, there the basic physical energy estimate is derived. Appendix C collects some auxiliary tools from analysis.

We use the usual notation for Lebesgue and Sobolev spaces as well as for derivatives with respect to space and time. The material time derivative of a scalar function $f$ is denoted by $\frac{D}{D t} f=f_{t}+\mathbf{v} \cdot \nabla f$. By $L_{\text {loc }}^{p}(\Omega), W_{\text {loc }}^{1, p}(\Omega)$, etc., we denote the local Lebesgue and Sobolev spaces, meaning that, e.g., $f \in L_{\text {loc }}^{p}(\Omega)$ if $f \in L^{p}(K)$ for any $K \subset \subset \Omega$. VMO means the space of functions with vanishing mean oscillation (see, e.g., [10]). Vector fields will be denoted in boldface, and we write $\mathcal{U}, \mathcal{V}, \mathcal{W}$ for the spaces $H_{0}^{1}\left(\Omega^{*}\right),\left\{\mathbf{v} \in\left(H_{0}^{1}(\Omega)\right)^{3} \mid \nabla \cdot \mathbf{v}=0\right\}$, and $H^{1}(\Omega)$, respectively. For a space $X$, we denote by $X^{\prime}$ its topological dual space. Generically, $\langle\cdot, \cdot\rangle$ denotes the $L^{2}$ scalar product; other duality pairings will be defined when needed. We abbreviate $x^{+}:=\max (x, 0)$.

We make the following general assumptions.

$\left(A_{1}\right)$ The domains $\Omega \subset \subset \Omega^{*} \subset \mathbb{R}^{3}$ are both open, bounded with $\partial \Omega \in C^{3}$ and $\partial \Omega^{*} \in C^{0,1}$; the final time $T>0$ is arbitrary but fixed. 
$\left(A_{2}\right)$ The dielectric parameter $\varepsilon(\cdot) \in C^{1,1}(\mathbb{R})$ satisfies $0<\varepsilon_{-} \leq \varepsilon(\phi) \leq \varepsilon_{+}<\infty$ for all $\phi \in \mathbb{R}$ and is monotone with $\operatorname{supp}\left(\varepsilon^{\prime}\right) \subset[-1,1]$. On $\Omega_{T}^{*}$, we consider the function

$$
\bar{\varepsilon}[\phi](t, x):= \begin{cases}\varepsilon(\phi(t, x)) & \text { if } x \in \bar{\Omega}, \\ \varepsilon_{*}(x) & \text { if } x \in \bar{\Omega}^{*} \backslash \bar{\Omega} .\end{cases}
$$

Here, $\varepsilon_{*} \in L^{\infty}\left(\Omega^{*} \backslash \Omega\right)$ is bounded from below by a positive parameter. We introduce $\Gamma(\cdot):=K(\cdot) / \varepsilon(\cdot)$, and we assume the recombination/dissociation term for simplicity ${ }^{1}$ to be $R\left(\rho_{1}, \rho_{2}, \rho_{0}, \phi\right):=K_{1}(\phi) \rho_{1}^{+} \rho_{2}^{+}-K_{0}(\phi)\left(\rho_{0}^{+}\right)^{\alpha}$ with $K_{1}(\cdot) \geq \Gamma(\cdot)$ and $\alpha \in(0,1)$.

$\left(A_{3}\right)$ The mobility $M(\cdot) \in C^{0,1}(\mathbb{R})$ is bounded from below by a positive constant $M_{-}$.

$\left(A_{4}\right)$ The interfacial energy $\gamma_{f s} \in C^{1,1}(\mathbb{R})$ and the viscosity function $\eta \in C^{0,1}(\mathbb{R})$ are monotone (increasing or decreasing) on $[-1,1]$ with $\operatorname{supp}\left(\gamma_{f s}^{\prime}\right) \subset[-1,1]$ and $\eta_{-} \leq \eta(\cdot) \leq \eta_{+}, \alpha(\cdot) \geq \alpha_{-}>0$ a.e. on $\partial \Omega, \alpha(\cdot) \in L^{\infty}(\partial \Omega)$.

$\left(A_{5}\right)$ The conductivity functions $K(\cdot), K_{1}(\cdot), K_{0}(\cdot) \in C^{1}(\mathbb{R})$ satisfy $0<K_{-} \leq$ $K_{i}(\cdot), K(\cdot) \leq K_{+}$for $i \in\{0,1\}$ and are monotone increasing with the additional property $\operatorname{supp}\left(K^{\prime}, K_{0}^{\prime}, K_{1}^{\prime}\right) \subset[-1,1]$.

$\left(A_{6}\right) \bar{V} \in W^{1,1}\left(I ; L^{\infty}\left(\Omega^{*}\right)\right) \cap L^{\infty}\left(I ; W^{1, r}\left(\Omega^{*}\right)\right) \cap H^{1}\left(I ; H^{1}\left(\Omega^{*}\right)\right)$ for an exponent $r>2$ and arbitrary time intervals $I$.

$\left(A_{7}\right) W(\cdot) \in C^{1,1}(\mathbb{R})$.

$\left(A_{8}\right) s>6$ ( $s$-Laplacian) (or $s>2$ in space dimension $d=2$; see also the remark after Lemma 3.2).

Note that $\left(A_{2}\right),\left(A_{4}\right)$, and $\left(A_{5}\right)$ put into simple mathematical terms the physical fact that we are dealing with a mixture of two different liquids, and therefore the physical quantities dielectricity, interfacial energy, viscosity, and conductivity depend on the phase field. We do not require $K(\cdot), K_{1}(\cdot)$, and $K_{0}(\cdot)$ to vanish in the phase corresponding to the ambient liquid, as a confinement of the ions to the electrolyte phase is not realistic from the physical point of view. $\left(A_{1}\right),\left(A_{3}\right)$, and $\left(A_{6}\right)-\left(A_{8}\right)$ are merely technical assumptions; in particular $\left(A_{8}\right)$ reflects the intricate coupling between $V, \rho_{i}, i \in\{1,2\}$, and $\phi$-see, for instance, the proof of Lemma 3.2. Now, we are able to state our main existence result, which we are going to prove in the following sections.

TheOrem 1.1 (main existence result). Let assumptions $\left(A_{1}\right)-\left(A_{8}\right)$ hold. For given initial data $\mathbf{v}_{0} \in \mathcal{V}, \rho_{i, 0} \in L_{\text {loc }}^{\infty}(\Omega) \cap L^{2}(\Omega)$ with $\rho_{i, 0} \geq 0$ a.e. in $\Omega$ for $i \in\{0,1,2\}$, $\phi_{0} \in W^{1, s}(\Omega)$, there exists a septuple $\left(\mathbf{v}, \rho_{0}, \rho_{1}, \rho_{2}, \phi, \mu, V\right)$ such that $\rho_{i} \geq 0$ a.e. in $\Omega_{T}$ for $i \in\{0,1,2\}$ and such that

$$
\begin{aligned}
\rho_{0}^{p_{1}}, \rho_{1}^{p_{2}}, \rho_{2}^{p_{2}} & \in L^{\infty}\left(I ; L^{2}\left(\Omega^{\prime}\right)\right) \cap L^{2}\left(I ; H^{1}\left(\Omega^{\prime}\right)\right), \\
\rho_{1}, \rho_{2} & \in L^{\infty}\left(I ; L^{1}(\Omega)\right) \cap W^{1,3 / 2}\left(I ;\left(W_{0}^{1,3}\left(\Omega^{\prime}\right)\right)^{\prime}\right), \\
\rho_{0} & \in L^{\infty}\left(I ; L^{1}(\Omega)\right) \cap W^{1,5 / 4}\left(I ;\left(H_{0}^{1}\left(\Omega^{\prime}\right)\right)^{\prime}\right), \\
\mathbf{v} & \in L^{\infty}\left(I ; L^{2}(\Omega)\right) \cap L^{2}(I ; \mathcal{V}) \cap W^{1,6 / 5}\left(I ;\left(H_{0}^{1}\left(\Omega^{\prime}\right)\right)^{\prime}\right), \\
\phi & \in L^{\infty}\left(I ; W^{1, s}(\Omega)\right) \cap H^{1}\left(I ; L^{2}(\partial \Omega)\right) \cap H^{1}\left(I ;\left(H^{1}(\Omega)\right)^{\prime}\right), \\
V-\bar{V} & \in L^{\infty}\left(I ; H_{0}^{1}\left(\Omega^{*}\right)\right),
\end{aligned}
$$

\footnotetext{
${ }^{1}$ More generally, we may allow terms of the form $R\left(\rho_{1}, \rho_{2}, \rho_{0}, \phi\right):=K_{1}(\phi) \rho_{1}^{+} \rho_{2}^{+}-K_{0}(\phi) g\left(\rho_{0}\right)$ such that $0 \leq g\left(\rho_{0}\right) \leq c\left(1+\rho_{0}^{+}\right)^{\alpha}, \alpha \in(0,1)$.
} 


$$
\begin{aligned}
& V \in L^{q}\left(I ; W^{1, q}\left(\Omega^{\prime}\right)\right), \\
& \mu \in L^{2}\left(I ; H^{1}(\Omega)\right)
\end{aligned}
$$

for any $\Omega^{\prime} \subset \subset \Omega, p_{1} \in\left(\frac{1-\alpha}{2}, \infty\right), p_{2} \in(0, \infty)$, and $q \in(0,16 / 3)$. This septuple solves the system (1.1), (1.2a), (1.2d), (1.2e), (1.3), (1.4) in the sense that for all $\Omega^{\prime} \subset \subset \Omega$ it holds that

$\left\langle\mathbf{v}_{t}, \mathbf{w}\right\rangle_{1}+\int_{\Omega_{T}^{\prime}}((\mathbf{v} \cdot \nabla) \mathbf{v} \cdot \mathbf{w}+\eta(\phi) \mathbf{T}(\mathbf{v}): \mathbf{T}(\mathbf{w}))+\int_{\Omega_{T}^{\prime}}\left(\left(\rho_{1}-\rho_{2}\right) \nabla V-\mu \nabla \phi\right) \cdot \mathbf{w}=0$,

$$
\left\langle\rho_{0, t}, \psi_{1}\right\rangle_{2}+\int_{\Omega_{T}^{\prime}}\left(K(\phi) \nabla \rho_{0}-\rho_{0} \mathbf{v}\right) \cdot \nabla \psi_{1}+\int_{\Omega_{T}^{\prime}}\left(K_{0}(\phi) \rho_{0}^{\alpha}-K_{1}(\phi) \rho_{1} \rho_{2}\right) \psi_{1}=0
$$

$$
\begin{gathered}
\left.\left\langle\rho_{i, t}, \psi_{2}\right\rangle_{3}+\int_{\Omega_{T}^{\prime}}\left(K(\phi)\left((-1)^{i+1} \rho_{i} \nabla V+\nabla \rho_{i}\right)\right)-\rho_{i} \mathbf{v}\right) \cdot \nabla \psi_{2} \\
+\int_{\Omega_{T}^{\prime}}\left(-K_{0}(\phi) \rho_{0}^{\alpha}+K_{1}(\phi) \rho_{1} \rho_{2}\right) \psi_{2}=0 \text { for } i \in\{1,2\}, \\
\left\langle\phi_{t}, \psi_{3}\right\rangle_{4}+\int_{\Omega_{T}}\left(\mathbf{v} \cdot \nabla \phi \psi_{3}+M(\phi) \nabla \mu \cdot \nabla \psi_{3}\right)=0, \\
\int_{\Omega_{T}} \mu \psi_{4}=\int_{\Omega_{T}}\left(|\nabla \phi|^{s-2} \nabla \phi \cdot \nabla \psi_{4}+W^{\prime}(\phi) \psi_{4}\right)-\frac{1}{2} \int_{\Omega_{T}} \varepsilon^{\prime}(\phi)|\nabla V|^{2} \psi_{4} \\
+\int_{0}^{T} \int_{\partial \Omega}\left(\alpha \phi_{t}+\gamma_{f s}^{\prime}(\phi)\right) \psi_{4}, \\
\int_{\Omega_{T}^{*}} \bar{\varepsilon}[\phi] \nabla V \cdot \nabla \chi=\int_{\Omega_{T}}\left(\rho_{1}-\rho_{2}\right) \chi
\end{gathered}
$$

for every $\mathbf{w} \in L^{6}\left(I ; H_{0}^{1}\left(\Omega^{\prime}\right)\right)$ such that $\nabla \cdot \mathbf{w}=0, \psi_{1} \in L^{5}\left(I ; H_{0}^{1}\left(\Omega^{\prime}\right)\right), \psi_{2} \in$ $L^{3}\left(I ; W_{0}^{1,3}\left(\Omega^{\prime}\right)\right), \psi_{3} \in L^{2}(I ; \mathcal{W}), \psi_{4} \in L^{s}\left(I ; W^{1, s}(\Omega)\right) \cap H^{1}\left(I ; L^{2}(\partial \Omega)\right)$, and $\chi \in$ $L^{2}(I ; \mathcal{U}) . \quad\langle\cdot, \cdot\rangle_{1}, \ldots,\langle\cdot, \cdot\rangle_{4}$ denote the respective duality pairings in $L^{6}\left(I ; H_{0}^{1}\left(\Omega^{\prime}\right)\right)$, $L^{5}\left(I ; H_{0}^{1}\left(\Omega^{\prime}\right)\right), L^{3}\left(I ; W_{0}^{1,3}\left(\Omega^{\prime}\right)\right)$, and $L^{2}\left(I ; H^{1}(\Omega)\right)$. In particular, the total species mass

$$
\int_{\Omega}\left[2 \rho_{0}(t, \cdot)+\rho_{1}(t, \cdot)+\rho_{2}(t, \cdot)\right]
$$

is constant in time.

Moreover, under the assumption that the solution obtained in Theorem 1.1 is sufficiently regular, it can be shown that the boundary conditions $(1.2 \mathrm{~b}),(1.2 \mathrm{c})$ are attained; see Remark 3.15 in section 3.

2. A viscoelastic, mobility regularized model. In this section, we prove existence results for a - as we call it - viscoelastic, mobility regularized auxiliary problem; see equations (2.1) for a weak formulation. It differs from system (1.1) with respect to three aspects. In the Navier-Stokes equations, the additional term $-\sigma_{1} \Delta \mathbf{v}_{t}$ is included; in the ion density equations, the mobility factor $\rho_{i}^{+}, i \in\{1,2\}$, is replaced by $\frac{1}{M_{0}^{\prime \prime}\left(\rho_{i}\right)}=\frac{\rho_{i}^{+}}{1+\sigma \rho_{i}^{+}}$; and in the recombination/dissociation term, the product $\rho_{1}^{+} \rho_{2}^{+}$ is substituted by a standard $L^{\infty}$-approximation. For the VEMR model, an energy 
estimate can be obtained rigorously which is independent of all the approximation parameters.

THEOREM 2.1 (existence for VEMR). Let assumptions $\left(A_{1}\right)-\left(A_{8}\right)$ hold. For given initial data $\mathbf{v}_{0} \in \mathcal{V}, \rho_{i, 0} \in L^{2}(\Omega)$ with $\rho_{i, 0} \geq 0$ a.e. in $\Omega$ for $i \in\{0,1,2\}, \phi_{0} \in W^{1, s}(\Omega)$, there exists a septuple $\left(\mathbf{v}, \rho_{0}, \rho_{1}, \rho_{2}, \phi, \mu, V\right)$ such that for $i \in\{0,1,2\}$ we have $\rho_{i} \geq 0$ a.e. in $\Omega_{T}$ and for any $\beta<\frac{5}{6}$

$$
\begin{aligned}
\mathbf{v} & \in L^{\infty}(I ; \mathcal{V}) \cap H^{1}(I ; \mathcal{V}), \\
\rho_{0} & \in L^{\infty}\left(I ; L^{1}(\Omega)\right) \cap L^{\infty}\left(I ; L^{2}(\Omega)\right) \cap L^{2}\left(I ; H^{1}(\Omega)\right) \cap H^{1}\left(I ;\left(H^{1}(\Omega)\right)^{\prime}\right), \\
\rho_{i} & \in L^{\infty}\left(I ; L^{1}(\Omega)\right) \cap L^{\infty}\left(I ; L^{2}(\Omega)\right) \cap L^{2}\left(I ; H^{1}(\Omega)\right) \cap H^{1}\left(I ;\left(H^{1}(\Omega)\right)^{\prime}\right) \\
& \quad \text { or } i \in\{1,2\}, \\
\phi & \in L^{\infty}\left(I ; W^{1, s}(\Omega)\right) \cap C^{0}\left(\bar{I} ; C^{0, \beta}(\bar{\Omega})\right) \cap H^{1}\left(I ; L^{2}(\partial \Omega)\right) \cap H^{1}\left(I ;\left(H^{1}(\Omega)\right)^{\prime}\right), \\
V-\bar{V} & \in L^{\infty}\left(I ; H_{0}^{1}\left(\Omega^{*}\right)\right), \\
\mu & \in L^{2}\left(I ; H^{1}(\Omega)\right) .
\end{aligned}
$$

The $L^{\infty}\left(I ; L^{1}(\Omega)\right)$-bounds for the $\rho_{i}$ 's depend only on the initial data. All the other bounds depend on $\sigma, \sigma_{1}, \Lambda_{1}$. The functions solve the weak system

$$
\begin{gathered}
\int_{\Omega_{T}}\left(\left(\mathbf{v}_{t}+(\mathbf{v} \cdot \nabla) \mathbf{v}\right) \cdot \mathbf{w}+\sigma_{1} \nabla \mathbf{v}_{t} \cdot \nabla \mathbf{w}+\eta(\phi) \mathbf{T}(\mathbf{v}): \mathbf{T}(\mathbf{w})\right) \\
\quad+\int_{\Omega_{T}}\left(\left(\frac{\rho_{1}}{1+\sigma \rho_{1}}-\frac{\rho_{2}}{1+\sigma \rho_{2}}\right) \nabla V-\mu \nabla \phi\right) \cdot \mathbf{w}=0 \\
\int_{\Omega_{T}} \rho_{0, t} \psi_{1}+\int_{\Omega_{T}}\left(K(\phi) \nabla \rho_{0}-\rho_{0} \mathbf{v}\right) \cdot \nabla \psi_{1} \\
\quad+\int_{\Omega_{T}}\left(K_{0}(\phi) \rho_{0}^{\alpha}-K_{1}(\phi) \bar{M}_{\Lambda_{1}}\left(\rho_{1}\right) \bar{M}_{\Lambda_{1}}\left(\rho_{2}\right)\right) \psi_{1}=0, \\
\int_{\Omega_{T}} \rho_{i, t} \psi_{2}+\int_{\Omega_{T}}\left(K(\phi) \frac{1}{M_{0}^{\prime \prime}\left(\rho_{i}\right)} \nabla\left((-1)^{i+1} V+M_{0}^{\prime}\left(\rho_{i}\right)\right)-\frac{\rho_{i}}{1+\sigma \rho_{i}} \mathbf{v}\right) \cdot \nabla \psi_{2} \\
+\int_{\Omega_{T}}\left(-K_{0}(\phi) \rho_{0}^{\alpha}+K_{1}(\phi) \rho_{1} \rho_{2}\right) \psi_{2}=0 \text { for } i \in\{1,2\} \\
\int_{\Omega_{T}}\left(\phi_{t}+\mathbf{v} \cdot \nabla \phi\right) \psi_{3}+\int_{\Omega_{T}} M(\phi) \nabla \mu \cdot \nabla \psi_{3}=0, \\
\mu \psi_{4}=\int_{\Omega_{T}}\left(|\nabla \phi|^{s-2} \nabla \phi \cdot \nabla \psi_{4}+W^{\prime}(\phi) \psi_{4}-\frac{1}{2} \varepsilon^{\prime}(\phi)|\nabla V|^{2} \psi_{4}\right) \\
\int_{\Omega_{T}}+\int_{0}^{T} \int_{\partial \Omega}\left(\alpha \phi_{t}+\gamma_{f s}^{\prime}(\phi)\right) \psi_{4}, \\
\int_{\Omega_{T}^{*}} \bar{\varepsilon}[\phi] \nabla V \cdot \nabla \chi=\int_{\Omega_{T}}\left(\rho_{1}-\rho_{2}\right) \chi
\end{gathered}
$$

for every $\mathbf{w} \in L^{2}(I ; \mathcal{V}), \psi_{1} \in L^{2}\left(I ; H^{1}(\Omega)\right), \psi_{2} \in L^{2}\left(I ; H^{1}(\Omega)\right) \cap L^{3}\left(\Omega_{T}\right), \psi_{3} \in$ $L^{2}\left(I ; H^{1}(\Omega)\right), \psi_{4} \in L^{s}\left(I ; W^{1, s}(\Omega)\right) \cap H^{1}\left(I ; L^{2}(\partial \Omega)\right)$, and $\chi \in L^{2}(I ; \mathcal{U})$. Here, $\sigma, \sigma_{1}<$ $1<\Lambda_{1}$ are approximation parameters, $\bar{M}_{\Lambda}(x)=\min (x, \Lambda)$, and

$$
M_{0}(\rho)= \begin{cases}\rho \log \rho-\rho+\frac{\sigma}{2} \rho^{2}, & \rho>0 \\ 0, & \rho=0 .\end{cases}
$$

Copyright $@$ by SIAM. Unauthorized reproduction of this article is prohibited. 
Proof. Our starting point is the energy estimate (B.14) formulated for the basic auxiliary solutions obtained in Theorem B.1. For ease of presentation, we omit here the explicit dependence on the approximation parameters. Abbreviating $\rho_{i, L}:=$ $\max \left(\rho_{i}, L\right)$, estimate (B.14) can be written in the form

$$
\begin{aligned}
& \underset{t \in(0, T)}{\operatorname{esssup}}\left[\int _ { \Omega } \left\{\frac{1}{2}|\mathbf{v}|^{2}+\frac{\sigma_{1}}{2}|\nabla \mathbf{v}|^{2}+M_{L}\left(\rho_{1}\right)+M_{L}\left(\rho_{2}\right)+\frac{1}{s}|\nabla \phi|^{s}\right.\right. \\
& \left.+W(\phi)\}+\int_{\partial \Omega} \gamma_{f s}(\phi)+\int_{\Omega^{*}} \frac{1}{2} \bar{\varepsilon}[\phi]|\nabla V|^{2}\right](t) \\
& +\int_{\Omega_{T}}\left[\eta(\phi)|\mathbf{T}(\mathbf{v})|^{2}+\sigma_{3}\left|V+M_{L}^{\prime}\left(\rho_{1}\right)\right|^{2}+\sigma_{3}\left|-V+M_{L}^{\prime}\left(\rho_{2}\right)\right|^{2}\right. \\
& +K(\phi) \frac{1}{M_{L}^{\prime \prime}\left(\rho_{1}\right)}\left|\nabla\left[V+M_{L}^{\prime}\left(\rho_{1}\right)\right]\right|^{2}+K(\phi) \frac{1}{M_{L}^{\prime \prime}\left(\rho_{2}\right)}\left|\nabla\left[-V+M_{L}^{\prime}\left(\rho_{2}\right)\right]\right|^{2} \\
& \left.+M(\phi)|\nabla \mu|^{2}\right]+\int_{\partial \Omega_{T}} \alpha\left|\phi_{t}\right|^{2} \\
& +\sigma_{2} \int_{\Omega_{T}} \frac{1}{\rho_{1, L}}\left|\nabla \rho_{1}\right|^{2}+\sigma_{2} \sigma \int_{\Omega_{T}}\left|\nabla \rho_{1}\right|^{2}+\sigma_{2} \int_{\Omega_{T}} \frac{1}{\rho_{2, L}}\left|\nabla \rho_{2}\right|^{2}+\sigma_{2} \sigma \int_{\Omega_{T}}\left|\nabla \rho_{2}\right|^{2} \\
& +\int_{\Omega_{T}} K_{1}(\phi) \bar{M}_{\Lambda_{2}}\left(\rho_{1}^{+}\right) \bar{M}_{\Lambda_{2}}\left(\rho_{2}^{+}\right)\left(\sigma \rho_{1}+\sigma \rho_{2}\right) \\
& +\int_{\left[\rho_{1} \geq 1\right]} K_{1}(\phi) \bar{M}_{\Lambda_{2}}\left(\rho_{1}^{+}\right) \bar{M}_{\Lambda_{2}}\left(\rho_{2}^{+}\right) \log \rho_{1}+\int_{\left[\rho_{2} \geq 1\right]} K_{1}(\phi) \bar{M}_{\Lambda_{2}}\left(\rho_{1}^{+}\right) \bar{M}_{\Lambda_{2}}\left(\rho_{2}^{+}\right) \log \rho_{2} \\
& +\int_{\left[\rho_{1} \leq L\right]} K_{1}(\phi) \bar{M}_{\Lambda_{2}}\left(\rho_{1}^{+}\right) \bar{M}_{\Lambda_{2}}\left(\rho_{2}^{+}\right) \frac{\rho_{1}}{L}+\int_{\left[\rho_{2} \leq L\right]} K_{1}(\phi) \bar{M}_{\Lambda_{2}}\left(\rho_{1}^{+}\right) \bar{M}_{\Lambda_{2}}\left(\rho_{2}^{+}\right) \frac{\rho_{2}}{L} \\
& -\int_{\left[L<\rho_{1}<1\right]} K_{0}(\phi)\left(\rho_{0}\right)^{\alpha} \log \rho_{1}-\int_{\left[L<\rho_{2}<1\right]} K_{0}(\phi)\left(\rho_{0}\right)^{\alpha} \log \rho_{2} \\
& -\int_{\left[\rho_{1} \leq L\right]} K_{0}(\phi)\left(\rho_{0}\right)^{\alpha}(\log L-1)-\int_{\left[\rho_{2} \leq L\right]} K_{0}(\phi)\left(\rho_{0}\right)^{\alpha}(\log L-1) \\
& \leq C\left(\mathbf{v}_{0}, \rho_{0,0}, \rho_{1,0}, \rho_{2,0}, \phi_{0}, V_{0}\right)-\int_{\Omega_{T}^{*}} \bar{\varepsilon}[\phi] \nabla V \nabla \bar{V}_{t}+\left[\int_{\Omega^{*}} \bar{\varepsilon}[\phi] \nabla V \nabla \bar{V}\right]_{0}^{T} \\
& +\int_{\Omega_{T}}\left(\rho_{1}-\rho_{2}\right) \bar{V}_{t}-\left[\int_{\Omega}\left(\rho_{1}-\rho_{2}\right) \bar{V}\right]_{0}^{T}-\sigma_{2} \int_{\Omega_{T}} \nabla V \cdot \nabla\left(\rho_{1}-\rho_{2}\right) \\
& -\int_{\left[L<\rho_{1}<1\right]} K_{1}(\phi) \bar{M}_{\Lambda_{2}}\left(\rho_{1}^{+}\right) \bar{M}_{\Lambda_{2}}\left(\rho_{2}^{+}\right) \log \rho_{1} \\
& -\int_{\left[L<\rho_{2}<1\right]} K_{1}(\phi) \bar{M}_{\Lambda_{2}}\left(\rho_{1}^{+}\right) \bar{M}_{\Lambda_{2}}\left(\rho_{2}^{+}\right) \log \rho_{2} \\
& -\int_{\left[\rho_{1} \leq L\right]} K_{1}(\phi) \bar{M}_{\Lambda_{2}}\left(\rho_{1}^{+}\right) \bar{M}_{\Lambda_{2}}\left(\rho_{2}^{+}\right)(\log L-1) \\
& -\int_{\left[\rho_{2} \leq L\right]} K_{1}(\phi) \bar{M}_{\Lambda_{2}}\left(\rho_{1}^{+}\right) \bar{M}_{\Lambda_{2}}\left(\rho_{2}^{+}\right)(\log L-1) \\
& +\int_{\left[\rho_{1} \geq 1\right]} K_{0}(\phi)\left(\rho_{0}\right)^{\alpha} \log \rho_{1}+\int_{\left[\rho_{2} \geq 1\right]} K_{0}(\phi)\left(\rho_{0}\right)^{\alpha} \log \rho_{2} \\
& +\int_{\Omega_{T}} K_{0}(\phi)\left(\rho_{0}\right)^{\alpha}\left(\sigma \rho_{1}+\sigma \rho_{2}\right)+\int_{\left[\rho_{1} \leq L\right]} K_{0}(\phi)\left(\rho_{0}\right)^{\alpha} \frac{\rho_{1}}{L}+\int_{\left[\rho_{2} \leq L\right]} K_{0}(\phi)\left(\rho_{0}\right)^{\alpha} \frac{\rho_{2}}{L} \\
& \leq \bar{C}\left(\mathbf{v}_{0}, \rho_{0,0}, \rho_{1,0}, \rho_{2,0}, \phi_{0}, V_{0}, \bar{V}\right) \text {. }
\end{aligned}
$$

Copyright $@$ by SIAM. Unauthorized reproduction of this article is prohibited. 
The bound on the right-hand side (r.h.s.) can be derived using Hölder's, Young's, and Gronwall's inequalities and some standard absorption techniques. It is independent of $\sigma_{2}, \sigma_{3}, \Lambda_{2}$, and $L$, but depends on $\sigma$ and $\Lambda_{1}$. Note further that the boundedness of

$$
\int_{\Omega_{T}} K_{1}(\phi) \bar{M}_{\Lambda_{2}}\left(\rho_{1}^{+}\right) \bar{M}_{\Lambda_{2}}\left(\rho_{2}^{+}\right)\left(\sigma \rho_{1}+\sigma \rho_{2}\right)
$$

implies $\bar{M}_{\Lambda_{2}}\left(\rho_{1}^{+}\right) \bar{M}_{\Lambda_{2}}\left(\rho_{2}^{+}\right) \in L^{3 / 2}\left(\Omega_{T}\right)$ to be uniformly bounded with a constant depending only on $\sigma$ and $\Lambda_{1}$. Moreover, combining the $L^{\infty}\left(L^{2}(\Omega)\right)$-estimate of $\nabla V$ with the $L^{1}\left(\Omega_{T}\right)$-boundedness of

$$
K(\phi) \frac{1}{M_{L}^{\prime \prime}\left(\rho_{i}\right)}\left|\nabla\left((-1)^{i+1} V+M_{L}^{\prime}\left(\rho_{i}\right)\right)\right|^{2}, \quad i=1,2,
$$

an $L^{2}\left(H^{1}(\Omega)\right.$ )-bound on $\rho_{i}, i=1,2$, which still depends on $\sigma$, can be established. By these estimates, compactness in time follows with techniques conceptually similar to those in the proof of Theorem B.1, this time the estimates depending on $\sigma, \sigma_{1}$, and $\Lambda_{1}$. Let $\mathbf{s}:=\left(\sigma_{2}, \sigma_{3}, \Lambda_{2}, L\right)^{T}$. We use (2.2) together with Rellich's imbedding theorem for $\mathbf{v}$, with a compactness result by Simon (see [27, Cor. 4, p. 85]) for $\phi$ and with the Aubin-Lions lemma for all the remaining quantities, to discuss the convergence behavior. For appropriate subsequences, we get the following for any $r<1, \beta<\frac{5}{6}$ and depending only on $\sigma, \sigma_{1}$, and $\Lambda_{1}$ :

$$
\begin{aligned}
& \mathbf{v}^{\mathbf{s}} \rightarrow \mathbf{v} \text { weakly in } H^{1}\left(\Omega_{T}\right)^{3}, \\
& \text { weakly * in } L^{\infty}\left(I ; H^{1}(\Omega)^{3}\right) \text {, } \\
& \text { and strongly in } H^{r}\left(\Omega_{T}\right)^{3} \text {, } \\
& \rho_{i}^{\mathbf{s}} \rightarrow \rho_{i} \text { weakly in } H^{1}\left(I ;\left(H^{1}(\Omega)\right)^{\prime}\right) \cap L^{2}\left(I ; H^{1}(\Omega)\right) \text {, } \\
& \text { weakly * in } L^{\infty}\left(I ; L^{2}(\Omega)\right) \text {, } \\
& \text { and strongly in } L^{2}\left(I ; H^{r}(\Omega)\right) \text { for } i \in\{0,1,2\} \text {, } \\
& \phi^{\mathbf{s}} \rightarrow \phi \text { weakly in } H^{1}\left(I ;\left(H^{1}(\Omega)\right)^{\prime}\right), \\
& \text { weakly * in } L^{\infty}\left(I ; W^{1, s}(\Omega)\right) \text {, } \\
& \text { and strongly in } C^{0}\left(\bar{I} ; C^{0, \beta}(\bar{\Omega})\right) \text {, } \\
& \phi^{\mathbf{s}} \rightarrow \phi \text { weakly in } H^{1}\left(I ; L^{2}(\partial \Omega)\right) \text {, } \\
& \text { and strongly in } L^{2}(\partial \Omega \times I) \text {, } \\
& \phi^{\mathbf{s}}(t, x) \rightarrow \phi(t, x) \text { for a.e. } t \in I, x \in \Omega, \\
& \nabla \mu^{\mathbf{s}} \rightarrow \nabla \mu \text { weakly in } L^{2}\left(\Omega_{T}\right), \\
& V^{\mathbf{s}} \stackrel{*}{\rightarrow} V \text { weakly } * \text { in } L^{\infty}\left(I ; H^{1}\left(\Omega^{*}\right)\right) \text {. }
\end{aligned}
$$

As in [11, p. 278], one can show

$$
V^{\mathbf{s}} \rightarrow V \text { strongly in } L^{2}\left(I ; H^{1}\left(\Omega^{*}\right)\right)
$$

Now, we pass with $\mathbf{s}:=\left(\sigma_{2}, \sigma_{3}, \Lambda_{2}, L\right)^{T}$ to the limit $(0,0, \infty, 0)^{T}$ in (B.1) to get (2.1). Note that the convergence in (B.1e) follows similarly to the convergence of (B.2e) at the end of Appendix B using the pseudomonotonicity of $-\Delta_{s}$. Before passing to the limit in the energy estimate (2.2), we prove nonnegativity for the charge densities. 
Therefore, test in (2.1b) by $\rho_{0}^{-}$to get

$$
\begin{aligned}
{\left[\int_{\Omega} \frac{1}{2}\left|\rho_{0}^{-}\right|^{2}\right]_{0}^{T} } & +\int_{\Omega_{T}} K(\phi)\left|\nabla \rho_{0}^{-}\right|^{2} \\
& +\int_{\Omega_{T}} K_{0}(\phi)\left|\rho_{0}^{-}\right|^{\alpha+1}-\int_{\Omega_{T}} K_{1}(\phi) \bar{M}_{\Lambda_{1}}\left(\rho_{1}^{+}\right) \bar{M}_{\Lambda_{1}}\left(\rho_{2}^{+}\right) \rho_{0}^{-}=0 .
\end{aligned}
$$

Noting that the last term on the left-hand side (l.h.s.) is nonnegative, we get $\rho_{0} \geq 0$ a.e. in $\Omega_{T}$. Similarly, one can show $\rho_{i} \geq 0$ for $i \in\{1,2\}$. Now, test in (2.1b) by $\psi_{1} \equiv 2$ and in (2.1c) by $\psi_{2} \equiv 1$ and add to get

$$
\left[\int_{\Omega} 2 \rho_{0}+\rho_{1}+\rho_{2}\right]_{0}^{T}+\int_{\Omega_{T}} 2 K_{1}(\phi)\left(\rho_{1}^{+} \rho_{2}^{+}-\bar{M}_{\Lambda_{1}}\left(\rho_{1}^{+}\right) \bar{M}_{\Lambda_{1}}\left(\rho_{2}^{+}\right)\right)=0 .
$$

Since $\rho_{i} \geq 0$ a.e. in $\Omega_{T}$ and since the second term on the l.h.s. is nonnegative, we get parameter-independent $L^{\infty}\left(I ; L^{1}(\Omega)\right)$-bounds for all the $\rho_{i}$ 's.

Next, we pass to the limit $\mathbf{s}=\left(\sigma_{2}, \sigma_{3}, \Lambda_{2}, L\right)^{T} \rightarrow(0,0, \infty, 0)^{T}$ in (2.2), using Fatou's lemma and the lower semicontinuity of the norm under weak convergence. We get for the limit $\left(\mathbf{v}, \rho_{0}, \rho_{1}, \rho_{2}, \phi, \mu, V\right)$ of appropriate subsequences

$$
\begin{aligned}
& \underset{t \in(0, T)}{\operatorname{esssup}}\left[\int _ { \Omega } \left\{\frac{1}{2}|\mathbf{v}|^{2}+\frac{\sigma_{1}}{2}|\nabla \mathbf{v}|^{2}+M_{0}\left(\rho_{1}\right)+M_{0}\left(\rho_{2}\right)+\frac{1}{s}|\nabla \phi|^{s}\right.\right. \\
& \left.+W(\phi)\}+\int_{\partial \Omega} \gamma_{f s}(\phi)+\int_{\Omega^{*}} \frac{1}{2} \bar{\varepsilon}[\phi]|\nabla V|^{2}\right](t) \\
& +\int_{\Omega_{T}}\left[\eta(\phi)|\mathbf{T}(\mathbf{v})|^{2}+K(\phi) \frac{1}{M_{0}^{\prime \prime}\left(\rho_{1}\right)}\left|\nabla\left[V+M_{0}^{\prime}\left(\rho_{1}\right)\right]\right|^{2}\right. \\
& \left.\quad+K(\phi) \frac{1}{M_{0}^{\prime \prime}\left(\rho_{2}\right)}\left|\nabla\left[-V+M_{0}^{\prime}\left(\rho_{2}\right)\right]\right|^{2}+M(\phi)|\nabla \mu|^{2}\right] \\
& \quad+\int_{\partial \Omega_{T}} \alpha\left|\phi_{t}\right|^{2}+\int_{\Omega_{T}} K_{1}(\phi) \rho_{1}^{+} \rho_{2}^{+}\left(\sigma \rho_{1}+\sigma \rho_{2}\right) \\
& \quad+\int_{\left[\rho_{1} \geq 1\right]} K_{1}(\phi) \rho_{1}^{+} \rho_{2}^{+} \log \rho_{1}+\int_{\left[\rho_{2} \geq 1\right]} K_{1}(\phi) \rho_{1}^{+} \rho_{2}^{+} \log \rho_{2} \\
& \quad-\int_{\left[0<\rho_{1}<1\right]} K_{0}(\phi)\left(\rho_{0}\right)^{\alpha} \log \rho_{1}-\int_{\left[0<\rho_{2}<1\right]} K_{0}(\phi)\left(\rho_{0}\right)^{\alpha} \log \rho_{2} \\
& \leq C\left(\mathbf{v}_{0}, \rho_{0,0}, \rho_{1,0}, \rho_{2,0}, \phi_{0}, V_{0}\right)-\int_{\Omega_{T}^{*}} \bar{\varepsilon}[\phi] \nabla V \nabla \bar{V}_{t}+\left[\int_{\Omega^{*}} \bar{\varepsilon}[\phi] \nabla V \nabla \bar{V}\right]_{0}^{T} \\
& \quad+\int_{\Omega_{T}}\left(\rho_{1}-\rho_{2}\right) \bar{V}_{t}-\left[\int_{\Omega}\left(\rho_{1}-\rho_{2}\right) \bar{V}\right]_{0}^{T}+\int_{\Omega_{T}} K_{0}(\phi)\left(\rho_{0}\right)^{\alpha}\left(\sigma \rho_{1}+\sigma \rho_{2}\right) \\
& \quad+\int_{\left[\rho_{1} \geq 1\right]} K_{0}(\phi)\left(\rho_{0}\right)^{\alpha} \log \rho_{1}+\int_{\left[\rho_{2} \geq 1\right]} K_{0}(\phi)\left(\rho_{0}\right)^{\alpha} \log \rho_{2} \\
& \quad-\int_{\left[0<\rho_{1}<1\right]} K_{1}(\phi) \rho_{1}^{+} \rho_{2}^{+} \log \rho_{1}-\int_{\left[0<\rho_{2}<1\right]} K_{1}(\phi) \rho_{1}^{+} \rho_{2}^{+} \log \rho_{2} \\
& \leq \bar{C}\left(\mathbf{v}_{0}, \rho_{0,0}, \rho_{1,0}, \rho_{2,0}, \phi_{0}, V_{0}, \bar{V}\right)
\end{aligned}
$$

by the parameter-independent $L^{\infty}\left(I ; L^{1}(\Omega)\right)$-boundedness of the $\rho_{i}$ 's and standard absorption estimates.

Copyright $\odot$ by SIAM. Unauthorized reproduction of this article is prohibited. 
With these bounds at hand, we are ready to prove higher regularity for the $\rho_{i}$ 's in the next section.

3. Higher regularity of the VEMR model and proof of the main existence result. In this section, we suggest an iteration procedure to improve regularity for the species equations to liberate ourselves from the approximation parameters $\sigma, \sigma_{1}$, and $\Lambda_{1}$ in the VEMR model. In the subsequent lemmas we use in the estimates of the terms on the r.h.s. the convention that the index $i$ is summed up over $i \in\{1,2\}$.

LEMma 3.1 (regularity for $\left.\rho_{0}(\mathrm{I})\right)$. Let $\left(\mathbf{v}, \rho_{0}, \rho_{1}, \rho_{2}, \phi, \mu, V\right)$ be a solution of (2.1). Then we have for any $p \in\left(\frac{1-\alpha}{2}, \frac{1}{2}\right)$ and any $q \in\left[1, \frac{5}{3}\right)$

$$
\rho_{0}^{p} \in L^{\infty}\left(I ; L^{2}(\Omega)\right) \cap L^{2}\left(I ; H^{1}(\Omega)\right), \quad \rho_{0} \in L^{q}\left(\Omega_{T}\right),
$$

independently of $\sigma, \sigma_{1}, \Lambda_{1}$.

Proof. Test in $(2.1 \mathrm{~b})$ by $\frac{1}{\beta} \rho_{0}^{\beta}, \beta \in(-\alpha, 0)$, to get

$$
\begin{aligned}
& \left|\frac{1}{\beta(\beta+1)}\right| \int_{\Omega} \rho_{0}^{\beta+1}(0)+\int_{\Omega_{T}} K(\phi) \rho_{0}^{\beta-1}\left|\nabla \rho_{0}\right|^{2}+\left|\frac{1}{\beta}\right| \int_{\Omega_{T}} K_{1}(\phi) \bar{M}_{\Lambda_{1}}\left(\rho_{1}^{+}\right) \bar{M}_{\Lambda_{1}}\left(\rho_{2}^{+}\right) \rho_{0}^{\beta} \\
& \quad=\left|\frac{1}{\beta(\beta+1)}\right| \int_{\Omega} \rho_{0}^{\beta+1}(T)+\left|\frac{1}{\beta}\right| \int_{\Omega_{T}} K_{0}(\phi) \rho_{0}^{\alpha+\beta} .
\end{aligned}
$$

This approach can be made rigorous as in [8] by testing first with $\frac{1}{\beta}\left(\rho_{0}+\varepsilon\right)^{\beta}$ for some $\varepsilon>0$ and then passing to the limit $\varepsilon \rightarrow 0$. The r.h.s. is obviously controlled by the uniform $L^{\infty}\left(I ; L^{1}(\Omega)\right)$-bound of $\rho_{0}$. As a consequence, $\rho_{0}^{(\beta+1) / 2} \in L^{2}\left(I ; H^{1}(\Omega)\right) \cap$ $L^{\infty}\left(I ; L^{2}(\Omega)\right)$ and by Lemma C.1, $\rho_{0} \in L^{\frac{5}{3}(\beta+1)}\left(\Omega_{T}\right)$ for any $\beta \in(-\alpha, 0)$.

Here is a first localized regularity result for $\rho_{1}, \rho_{2}$.

Lemma 3.2 (q-entropy). Let $\left(\mathbf{v}, \rho_{0}, \rho_{1}, \rho_{2}, \phi, \mu, V\right)$ be a solution of (2.1). Then we have for $i \in\{1,2\}$, any $q \in(-1,0)$, and arbitrary $\Omega^{\prime} \subset \subset \Omega$

$$
\rho_{i}^{(q+1) / 2} \in L^{\infty}\left(I ; L^{2}\left(\Omega^{\prime}\right)\right) \cap L^{2}\left(I ; H^{1}\left(\Omega^{\prime}\right)\right), \quad \rho_{i}^{2+q} \in L^{1}\left(I ; L^{1}\left(\Omega^{\prime}\right)\right),
$$

with constants depending only on $\operatorname{dist}\left(\Omega^{\prime}, \partial \Omega\right)$ and the data, but not on $\sigma, \sigma_{1}$, and $\Lambda_{1}$.

Proof. Note that by binomial decomposition of

$$
\int_{\Omega_{T}} K(\phi)\left[\frac{1}{M_{0}^{\prime \prime}\left(\rho_{1}\right)}\left|\nabla\left[V+M_{0}^{\prime}\left(\rho_{1}\right)\right]\right|^{2}+\frac{1}{M_{0}^{\prime \prime}\left(\rho_{2}\right)}\left|\nabla\left[-V+M_{0}^{\prime}\left(\rho_{2}\right)\right]\right|^{2}\right]
$$

in (2.5), $\nabla \rho_{1}, \nabla \rho_{2} \in L^{2}\left(I ; L^{2}(\Omega)\right)$, at this stage depending on $\sigma$. As a test function in (2.1c), we choose $\psi_{2}=\Psi^{n}\left(\frac{1}{q} \rho_{i}^{q}+\frac{\sigma}{1+q} \rho_{i}^{q+1}\right)$ for $i \in\{1,2\}, \Psi \in C_{0}^{\infty}(\Omega ;[0,1])$ such that $\left.\Psi\right|_{\Omega^{\prime}} \equiv 1, q \in(-1,0), n \geq 4$, and add the equations. This test function is of a similar type to that in Lemma 3.1, and hence a rigorous proof proceeds along the lines of [8]. Decomposing the elliptic term and inserting (2.1f), we get

$$
\begin{aligned}
& \left|\frac{1}{q(q+1)}\right| \int_{\Omega} \Psi^{n}\left(\rho_{1}^{q+1}(0)+\rho_{2}^{q+1}(0)\right)+\frac{\sigma}{(q+1)(q+2)} \int_{\Omega} \Psi^{n}\left(\rho_{1}^{q+2}(T)+\rho_{2}^{q+2}(T)\right) \\
& \quad+\int_{\Omega_{T}} \Psi^{n} K(\phi)\left[\rho_{1}^{q-1}\left|\nabla \rho_{1}\right|^{2}+\rho_{2}^{q-1}\left|\nabla \rho_{2}\right|^{2}+\sigma \rho_{1}^{q}\left|\nabla \rho_{1}\right|^{2}+\sigma \rho_{2}^{q}\left|\nabla \rho_{2}\right|^{2}\right] \\
& \quad+\left|\frac{1}{q}\right| \int_{\Omega_{T}} \Psi^{n} K_{0}(\phi) \rho_{0}^{\alpha}\left(\rho_{1}^{q}+\rho_{2}^{q}\right)+\frac{\sigma}{1+q} \int_{\Omega_{T}} \Psi^{n} K_{1}(\phi)\left(\rho_{1}^{q+2} \rho_{2}+\rho_{1} \rho_{2}^{q+2}\right) \\
& \quad+\frac{1}{q+1} \int_{\Omega_{T}} \Psi^{n} \Gamma(\phi)\left(\rho_{1}^{q+2}+\rho_{2}^{q+2}\right)
\end{aligned}
$$

Copyright $@$ ㅇ by SIAM. Unauthorized reproduction of this article is prohibited. 


$$
\begin{aligned}
=\mid & \frac{1}{q(q+1)} \mid \int_{\Omega} \Psi^{n}\left(\rho_{1}^{q+1}(T)+\rho_{2}^{q+1}(T)\right)+\frac{\sigma}{(q+1)(q+2)} \int_{\Omega} \Psi^{n}\left(\rho_{1}^{q+2}(0)+\rho_{2}^{q+2}(0)\right) \\
& +\frac{\sigma}{q+1} \int_{\Omega_{T}} \Psi^{n} K_{0}(\phi) \rho_{0}^{\alpha}\left(\rho_{1}^{q+1}+\rho_{2}^{q+1}\right)+\left|\frac{1}{q}\right| \int_{\Omega_{T}} \Psi^{n} K_{1}(\phi)\left(\rho_{1}^{q+1} \rho_{2}+\rho_{1} \rho_{2}^{q+1}\right) \\
& -\int_{\Omega_{T}} \Psi^{n} \mathbf{v} \cdot\left[\nabla\left(\frac{\rho_{1}}{1+\sigma \rho_{1}}\right)\left(\frac{1}{q} \rho_{1}^{q}+\frac{\sigma}{q+1} \rho_{1}^{q+1}\right)+\nabla\left(\frac{\rho_{2}}{1+\sigma \rho_{2}}\right)\left(\frac{1}{q} \rho_{2}^{q}+\frac{\sigma}{q+1} \rho_{2}^{q+1}\right)\right] \\
& +\frac{1}{q+1} \int_{\Omega_{T}} \Psi^{n} \Gamma(\phi)\left(\rho_{1}^{q+1} \rho_{2}+\rho_{1} \rho_{2}^{q+1}\right) \\
& +\frac{1}{q+1} \int_{\Omega_{T}} \Psi^{n} \Gamma^{\prime}(\phi) \nabla \phi \cdot \varepsilon(\phi) \nabla V\left(\rho_{1}^{q+1}-\rho_{2}^{q+1}\right) \\
& -\frac{n}{q+1} \int_{\Omega_{T}} \Psi^{n-1} K(\phi) \nabla \Psi \cdot\left[\rho_{1}^{q} \nabla \rho_{1}+\rho_{2}^{q} \nabla \rho_{2}+\sigma \rho_{1}^{q+1} \nabla \rho_{1}+\sigma \rho_{2}^{q+1} \nabla \rho_{2}\right] \\
& -\frac{n}{q(q+1)} \int_{\Omega_{T}} \Psi^{n-1} K(\phi) \nabla \Psi \cdot \nabla V\left(\frac{\rho_{1}^{q+1}}{1+\sigma \rho_{1}}-\frac{\rho_{2}^{q+1}}{1+\sigma \rho_{2}}\right) \\
& -\frac{n}{q(q+1)} \int_{\Omega_{T}} \Psi^{n-1} K(\phi) \nabla \Psi \cdot\left(\rho_{1}^{q} \nabla \rho_{1}+\rho_{2}^{q} \nabla \rho_{2}\right) \\
=R_{1} & +\cdots+R_{10} .
\end{aligned}
$$

Here, we used $\frac{1}{q} \rho_{i}^{q}+\frac{\sigma}{1+q} \rho_{i}^{q+1}=\frac{\rho_{i}^{q}}{q+1}\left(1+\sigma \rho_{i}\right)+\frac{1}{q(q+1)} \rho_{i}^{q}$. To estimate the r.h.s., note first that $\left|R_{1}\right|+\left|R_{2}\right| \leq C$ by $\rho_{i} \in L^{\infty}\left(I ; L^{1}(\Omega)\right), i \in\{1,2\}$, and the local boundedness of initial data. Moreover,

$$
\left|R_{3}\right| \leq C \sigma \int_{\Omega_{T}} \Psi^{n} \rho_{0}^{\alpha} \rho_{i}^{q+1} \leq C \int_{0}^{T}\left(\int_{\Omega} \rho_{0}^{2 \alpha}\right)^{1 / 2}\left(\sigma^{q+1} \int_{\Omega} \rho_{i}^{2(q+1)}\right)^{1 / 2} .
$$

Since $q<0$, the second integrand on the r.h.s. can be controlled by the $L^{\infty}\left(L^{1}\right)$-bound on $M_{0}\left(\rho_{i}\right)$, which implies $\sigma^{1 / 2} \rho_{i} \in L^{\infty}\left(I ; L^{2}(\Omega)\right)$ for $i \in\{1,2\}$ (cf. the energy estimate $(2.5))$. If $\alpha \in\left(0, \frac{1}{2}\right]$, the first integrand is bounded due to $\rho_{0} \in L^{\infty}\left(I ; L^{1}(\Omega)\right)$. For $\alpha \in\left(\frac{1}{2}, 1\right)$, we use Lemma C. 4 to estimate

$$
\int_{0}^{T}\left(\int_{\Omega} \rho_{0}^{2 \alpha}\right)^{1 / 2} \leq C \int_{\Omega_{T}}\left(\left|\rho_{0}^{\alpha / 2}\right|^{2}+\left|\nabla \rho_{0}^{\alpha / 2}\right|^{2}\right),
$$

which is bounded since $\rho_{0}^{p} \in L^{\infty}\left(I ; L^{2}(\Omega)\right) \cap L^{2}\left(I ; H^{1}(\Omega)\right)$ for any $p \in\left(\frac{1-\alpha}{2}, \frac{1}{2}\right)$ due to Lemma 3.1. In addition,

$$
\left|R_{4}\right|+\left|R_{6}\right| \leq C\left(\int_{\left[\rho_{1} \leq 1\right]} \rho_{2}+\int_{\left[\rho_{2} \leq 1\right]} \rho_{1}+\int_{\left[\rho_{1}>1\right]} \rho_{1} \rho_{2}+\int_{\left[\rho_{2}>1\right]} \rho_{1} \rho_{2}\right) \leq C
$$

due to the $L^{\infty}\left(I ; L^{1}(\Omega)\right)$-bounds for $\rho_{i}, i \in\{1,2\}$, and $\int_{\Omega_{T}} \rho_{1} \rho_{2}<\infty$ (test by $\psi_{2} \equiv 1$ in $(2.1 \mathrm{c}))$. Furthermore

$$
\begin{aligned}
\left|R_{5}\right| \leq\left|n \int_{\Omega_{T}} \Psi^{n-1} \nabla \Psi \cdot \mathbf{v}\left(\frac{1}{q\left(1+\sigma \rho_{i}\right)} \rho_{i}^{q+1}+\frac{\sigma \rho_{i}}{(q+1)\left(1+\sigma \rho_{i}\right)} \rho_{i}^{q+1}\right)\right| \\
\quad+\left|\int_{\Omega_{T}} \Psi^{n} \mathbf{v} \cdot\left(\frac{1}{1+\sigma \rho_{i}} \rho_{i}^{q} \nabla \rho_{i}+\frac{\sigma \rho_{i}}{1+\sigma \rho_{i}} \rho_{i}^{q} \nabla \rho_{i}\right)\right| \\
=\cdots+\left|n \int_{\Omega_{T}} \Psi^{n-1} \nabla \Psi \cdot \mathbf{v}\left(\int_{A}^{\rho_{i}} \frac{s^{q}}{1+\sigma s} d s+\int_{A}^{\rho_{i}} \frac{\sigma s^{q+1}}{1+\sigma s} d s\right)\right| .
\end{aligned}
$$

Copyright (c) by SIAM. Unauthorized reproduction of this article is prohibited. 
Now, estimate for $i \in\{1,2\}$

$$
\int_{\Omega_{T}} \Psi^{n-1} \nabla \Psi \cdot \mathbf{v} \rho_{i}^{q+1} \leq C_{\Psi, \delta} \int_{\Omega_{T}}|\mathbf{v}|^{10 / 3}+\delta \int_{\Omega_{T}} \Psi^{\frac{10}{7}(n-1)} \rho_{i}^{\frac{10}{7}(q+1)},
$$

and estimate the other terms analogously and absorb the $\delta$-terms within the $L^{q+2}$ terms on the l.h.s. This is possible as long as $q \leq \frac{4}{3}$. Furthermore,

$$
\begin{aligned}
\left|R_{7}\right| & \leq C \int_{\Omega_{T}} \Psi^{n}|\nabla \phi||\nabla V| \rho_{i}^{q+1} \\
& \leq C \int_{0}^{T}\left(\int_{\Omega}|\nabla \phi|^{s}\right)^{1 / s}\left(\int_{\Omega}|\nabla V|^{2}\right)^{1 / 2}\left(\int_{\Omega} \Psi^{p n} \rho_{i}^{p(q+1)}\right)^{1 / p} \\
& \leq C \int_{0}^{T}\left(\int_{\Omega} \Psi^{p n} \rho_{i}^{p(q+1)}\right)^{1 / p}=C \int_{0}^{T}\left(\int_{\Omega}\left(\Psi^{n / 2} \rho_{i}^{(q+1) / 2}\right)^{2 p}\right)^{2 / 2 p}
\end{aligned}
$$

with $p=\frac{2 s}{s-2}<3$, since $s>6$. Using Lemma C.4 again and recalling that $2 p<6$,

$$
\int_{0}^{T}\left(\int_{\Omega}\left(\Psi^{\frac{n}{2}} \rho_{i}^{\frac{q+1}{2}}\right)^{2 p}\right)^{\frac{2}{2 p}} \leq \delta \int_{\Omega_{T}} \Psi^{n} \rho_{i}^{q-1}\left|\nabla \rho_{i}\right|^{2}+C_{\delta}\left(\int_{\Omega_{T}} \Psi^{n} \rho_{i}^{q+1}+\int_{\Omega_{T}} \Psi^{n-2} \rho_{i}^{q+1}\right) .
$$

Now, the $\delta$-term can be absorbed on the l.h.s., and the other two terms are bounded due to $\rho_{i} \in L^{\infty}\left(I ; L^{1}(\Omega)\right)$ for $i \in\{1,2\}$. Further,

$$
\begin{aligned}
\left|R_{8}\right| & +\left|R_{10}\right| \leq \delta_{1} \int_{\Omega_{T}} \Psi^{n} K(\phi) \rho_{i}^{q-1}\left|\nabla \rho_{i}\right|^{2}+\delta_{2} \int_{\Omega_{T}} \Psi^{n} \Gamma(\phi) \rho_{i}^{q+2} \\
& +\delta_{3} \sigma \int_{\Omega_{T}} \Psi^{n} K(\phi) \rho_{i}^{q}\left|\nabla \rho_{i}\right|^{2}+\sigma C_{\delta_{3}} \int_{\Omega_{T}} \rho_{i}^{q+2}+C_{\Gamma, \delta_{1}, \delta_{2}} \int_{\Omega_{T}} \Psi^{n-2(2+q)},
\end{aligned}
$$

where the second-to-last term on the r.h.s. is bounded due to the energy estimate (2.5). For the last term on the r.h.s., $n \geq 4$ is needed. Finally,

$$
\left|R_{9}\right| \leq \delta \int_{\Omega_{T}} \Psi^{n} \Gamma(\phi) \rho_{i}^{q+2}+C_{\delta, \Psi} \int_{\Omega_{T}} \Psi^{n-2-q}|\nabla V|^{2+q},
$$

and the second term on the r.h.s. is readily estimated by the $L^{\infty}\left(L^{2}\right)$-bound on $\nabla V$.

Remark. Note that in space dimension $d=2$ only $s>2$ is needed to estimate the term $R_{7}$ on the r.h.s. above.

LEMma 3.3 (higher integrability for $\nabla V$ ). Let $\frac{6}{5}<q<3, s>6$, and $\Omega^{\prime} \subset \subset \Omega$. Further, let $\rho_{1}, \rho_{2} \in L^{p}\left(I ; L^{q}\left(\Omega^{\prime}\right)\right), \nabla V \in L^{\infty}\left(I ; L^{2}(\Omega)\right) \cap L^{p}\left(I ; L^{q}\left(\Omega^{\prime}\right)\right)$, and $\phi \in$ $L^{\infty}\left(I ; W^{1, s}(\Omega)\right)$. Then

$$
\nabla V \in L^{p}\left(I ; L^{3 q /(3-q)}\left(\Omega^{\prime}\right)\right)
$$

Proof. Let $\Psi \in C_{0}^{\infty}\left(\Omega^{\prime}\right)$. Obviously,

$$
\begin{aligned}
\nabla \cdot(\varepsilon(\phi) \nabla(\Psi V)) & =\Psi \nabla \cdot(\varepsilon(\phi) \nabla V)+\varepsilon(\phi) \nabla V \cdot \nabla \Psi+\nabla \cdot(\varepsilon(\phi) V \nabla \Psi) \\
& =-\Psi\left(\rho_{1}-\rho_{2}\right)+\varepsilon(\phi) \nabla V \cdot \nabla \Psi+\nabla \cdot(\varepsilon(\phi) V \nabla \Psi) \\
& \in L^{p}\left(I ; L^{q}\left(\Omega^{\prime}\right)\right) .
\end{aligned}
$$

Copyright (c) by SIAM. Unauthorized reproduction of this article is prohibited. 
Let $w$ be defined as the Newton potential of the r.h.s. Then $w \in L^{p}\left(I ; W^{2, q}\left(\Omega^{\prime}\right)\right)$. Hence, $\nabla w \in L^{p}\left(I ; W^{1, q}\left(\Omega^{\prime}\right)\right)$ and by Sobolev embedding also in $L^{p}\left(I ; L^{3 q /(3-q)}\left(\Omega^{\prime}\right)\right)$. Now, Di Fazio's Lemma C.5 entails the result.

An application of Lemma C.3 yields the following.

Corollary 3.4. $\nabla V \in L^{q}\left(I ; L^{q}\left(\Omega^{\prime}\right)\right)$ for any $q<10 / 3$ and any $\Omega^{\prime} \subset \subset \Omega$ independently of the approximation parameters.

The regularity results established so far are still not sufficient to prove existence of solutions in the spirit of the solution concept presented in Theorem 1.1. To proceed further, we have to use arbitrary powers of $\rho_{0}, \rho_{1}, \rho_{2}$ as test functions. To guarantee their admissibility, higher regularity results depending on the approximation parameters will be sufficient.

LEMMA 3.5 (approximation parameter-dependent regularity of $\rho_{0}$ ). Let $\Omega^{\prime} \subset \subset$ $\Omega$ be arbitrary and let $\rho_{0}^{\left(r_{1}+1\right) / 2} \in L^{\infty}\left(I ; L^{2}\left(\Omega^{\prime}\right)\right) \cap L^{2}\left(I ; H^{1}\left(\Omega^{\prime}\right)\right)$ for some $r_{1}>0$ (possibly depending on $\sigma, \sigma_{1}$, and $\Lambda_{1}$ ). Then there exists $\theta>1$ such that for $r_{2} \in$ $\left[r_{1}, \theta r_{1}\right]$

$$
\rho_{0}^{\left(r_{2}+1\right) / 2} \in L^{\infty}\left(I ; L^{2}\left(\Omega^{\prime}\right)\right) \cap L^{2}\left(I ; H^{1}\left(\Omega^{\prime}\right)\right), \quad \rho_{0}^{\frac{5}{3}\left(r_{2}+1\right)} \in L^{1}\left(I ; L^{1}\left(\Omega^{\prime}\right)\right),
$$

where the constants depend on $\operatorname{dist}\left(\Omega^{\prime}, \partial \Omega\right)$, the data, $\sigma, \sigma_{1}$, and $\Lambda_{1}$. By iteration, any $r_{2}<\infty$ can be achieved in finitely many steps.

Remark. From Theorem 2.1 and Lemma 3.1 we infer that the required $L^{2}\left(H^{1}\right)$ property of $\rho_{0}$ holds true for all $r_{1} \in(0,1]$.

Proof of Lemma 3.5. This result can be established by techniques similar to those in the subsequent Lemma 3.7. Note in particular that the estimates are not influenced by the integrability of $\rho_{1}$ and $\rho_{2}$ since on this level the cut-off terms $\bar{M}_{\Lambda_{1}}(\cdot)$ are still used.

Lemma 3.6 (approximation parameter-dependent integrability of $\nabla V$ ). It holds that

$$
\nabla V \in L^{\infty}\left(I ; L^{2}(\Omega)\right) \cap L^{q}\left(I ; L^{q}\left(\Omega^{\prime}\right)\right)
$$

for any $\Omega^{\prime} \subset \subset \Omega$ and any $q<16 / 3$ depending on $\sigma, \sigma_{1}$, and $\Lambda_{1}$.

Proof. This result can be proved analogously to Corollary 3.4.

LEMma 3.7 (approximation parameter-dependent regularity of $\rho_{i}$ ). Let $\Omega^{\prime} \subset \subset \Omega$ be arbitrary and let $\rho_{i}^{\left(m_{1}+2\right) / 2} \in L^{\infty}\left(I ; L^{2}\left(\Omega^{\prime}\right)\right) \cap L^{2}\left(I ; H^{1}\left(\Omega^{\prime}\right)\right), i \in\{1,2\}$, for some $m_{1} \geq 0$ (possibly depending on $\sigma, \sigma_{1}$, and $\Lambda_{1}$ ). Then there exists $\theta>1$ such that for $m_{2} \in\left[m_{1}, \theta m_{1}\right]$ and $i \in\{1,2\}$

$$
\rho_{i}^{\left(m_{2}+2\right) / 2} \in L^{\infty}\left(I ; L^{2}\left(\Omega^{\prime}\right)\right) \cap L^{2}\left(I ; H^{1}\left(\Omega^{\prime}\right)\right), \quad \rho_{i}^{\frac{5}{3}\left(m_{2}+2\right)} \in L^{1}\left(I ; L^{1}\left(\Omega^{\prime}\right)\right),
$$

where the constants depend on $\operatorname{dist}\left(\Omega^{\prime}, \partial \Omega\right)$, the data, $\sigma, \sigma_{1}$, and $\Lambda_{1}$. By iteration, any $m_{2}<\infty$ can be achieved in finitely many steps.

Proof. Let $K>0, \Psi \in C_{0}^{\infty}(\Omega,[0,1]),\left.\Psi\right|_{\Omega^{\prime}} \equiv 1$, and $n \geq 3$. Choose

$\psi_{2, K}=\Psi^{n}\left\{\frac{1}{m_{2}} \rho_{i}^{\frac{m_{1}+2}{2}} \min \left(\rho_{i}, K\right)^{m_{2}-\frac{m_{1}+2}{2}}+\sigma \frac{1}{m_{2}+1} \rho_{i}^{\frac{m_{1}+2}{2}} \min \left(\rho_{i}, K\right)^{m_{2}+1-\frac{m_{1}+2}{2}}\right\}$

as test function in (2.1c). Abbreviating $\rho_{i, K}:=\min \left(\rho_{i}, K\right)$, we get for $i \in\{1,2\}$

Copyright (c) by SIAM. Unauthorized reproduction of this article is prohibited. 


$$
\begin{aligned}
& \frac{1}{m_{2}\left(m_{2}+1\right)} \int_{\Omega} \Psi^{n} \rho_{i, K}^{m_{2}+1}(T)+\frac{\sigma}{\left(m_{2}+1\right)\left(m_{2}+2\right)} \int_{\Omega} \Psi^{n} \rho_{i, K}^{m_{2}+2}(T) \\
& +\int_{\left[\rho_{i}(T)>K\right]} \Psi^{n} K^{m_{2}-\frac{m_{1}+2}{2}}\left[\frac{1}{m_{2}} \frac{2}{m_{1}+4}\left(\rho_{i}^{\left(m_{1}+4\right) / 2}(T)-K^{\left(m_{1}+4\right) / 2}\right)\right. \\
& \left.+\frac{\sigma K}{m_{2}+1} \frac{2}{m_{1}+4}\left(\rho_{i}^{\left(m_{1}+4\right) / 2}(T)-K^{\left(m_{1}+4\right) / 2}\right)\right] \\
& +\int_{\left[\rho_{i} \leq K\right]} \Psi^{n} K(\phi)\left(\rho_{i}^{m_{2}-1}+\sigma \rho_{i}^{m_{2}}\right)\left|\nabla \rho_{i}\right|^{2} \\
& +\int_{\left[\rho_{i}>K\right]} \Psi^{n} K(\phi) K^{m_{2}-\frac{m_{1}+2}{2}}\left(\frac{m_{1}+2}{2 m_{2}} \rho_{i}^{m_{1} / 2}+\frac{\sigma K}{m_{2}+1} \frac{m_{1}+2}{2} \rho_{i}^{m_{1} / 2}\right)\left|\nabla \rho_{i}\right|^{2} \\
& +\frac{1}{m_{2}+1} \int_{\Omega_{T}} \Psi^{n} K_{1}(\phi) \rho_{1} \rho_{2} \rho_{i, K}^{m_{2}-\frac{m_{1}+2}{2}}\left(\frac{1}{m_{2}} \rho_{i}^{\left(m_{1}+2\right) / 2}+\rho_{i}^{\left(m_{1}+2\right) / 2}\left(1+\sigma \rho_{i, K}\right)\right) \\
& =\frac{1}{m_{2}\left(m_{2}+1\right)} \int_{\Omega} \Psi^{n} \rho_{i, K}^{m_{2}+1}(0)+\frac{\sigma}{\left(m_{2}+1\right)\left(m_{2}+2\right)} \int_{\Omega} \Psi^{n} \rho_{i, K}^{m_{2}+2}(0) \\
& +\int_{\left[\rho_{i}(0)>K\right]} \Psi^{n} K^{m_{2}-\frac{m_{1}+2}{2}}\left[\frac{1}{m_{2}} \frac{2}{m_{1}+4}\left(\rho_{i}^{\left(m_{1}+4\right) / 2}(0)-K^{\left(m_{1}+4\right) / 2}\right)\right. \\
& \left.+\frac{\sigma K}{m_{2}+1} \frac{2}{m_{1}+4}\left(\rho_{i}^{\left(m_{1}+4\right) / 2}(0)-K^{\left(m_{1}+4\right) / 2}\right)\right] \\
& +\frac{1}{m_{2}+1} \int_{\Omega_{T}} \Psi^{n} K_{0}(\phi) \rho_{0}^{\alpha} \rho_{i, K}^{m_{2}-\frac{m_{1}+2}{2}}\left(\frac{1}{m_{2}} \rho_{i}^{\left(m_{1}+2\right) / 2}+\rho_{i}^{\left(m_{1}+2\right) / 2}\left(1+\sigma \rho_{i, K}\right)\right) \\
& -\frac{n}{m_{2}+1} \int_{\Omega_{T}} \Psi^{n-1} K(\phi) \nabla \Psi \cdot \nabla \rho_{i} \rho_{i, K}^{m_{2}-\frac{m_{1}+2}{2}}\left(\frac{1}{m_{2}} \rho_{i}^{\left(m_{1}+2\right) / 2}+\rho_{i}^{\left(m_{1}+2\right) / 2}\left(1+\sigma \rho_{i, K}\right)\right) \\
& +(-1)^{i} \int_{\left[\rho_{i} \leq K\right]} \Psi^{n} K(\phi) \frac{\rho_{i}}{1+\sigma \rho_{i}} \nabla V \cdot\left(\rho_{i}^{m_{2}-1}+\sigma \rho_{i}^{m_{2}}\right) \nabla \rho_{i} \\
& +(-1)^{i} \int_{\left[\rho_{i}>K\right]} \Psi^{n} K(\phi) \frac{\rho_{i}}{1+\sigma \rho_{i}} \nabla V \cdot K^{m_{2}-\frac{m_{1}+2}{2}}\left(\frac{m_{1}+2}{2 m_{2}} \rho_{i}^{m_{1} / 2}+\frac{\sigma K}{m_{2}+1} \frac{m_{1}+2}{2} \rho_{i}^{m_{1} / 2}\right) \nabla \rho_{i} \\
& +\frac{(-1)^{i} n}{m_{2}+1} \int_{\Omega_{T}} \Psi^{n-1} K(\phi) \nabla \Psi \cdot \nabla V \frac{\rho_{i}}{1+\sigma \rho_{i}} \rho_{i, K}^{m_{2}-\frac{m_{1}+2}{2}}\left(\frac{1}{m_{2}} \rho_{i}^{\left(m_{1}+2\right) / 2}+\rho_{i}^{\left(m_{1}+2\right) / 2}\left(1+\sigma \rho_{i, K}\right)\right) \\
& -\frac{1}{m_{2}+1} \int_{\Omega_{T}} \Psi^{n} \mathbf{v} \cdot \nabla\left(\frac{\rho_{i}}{1+\sigma \rho_{i}}\right) \rho_{i, K}^{m_{2}-\frac{m_{1}+2}{2}}\left(\frac{1}{m_{2}} \rho_{i}^{\left(m_{1}+2\right) / 2}+\rho_{i}^{\left(m_{1}+2\right) / 2}\left(1+\sigma \rho_{i, K}\right)\right) \\
& =R_{1}+\cdots+R_{9} \text {. }
\end{aligned}
$$

Estimating the r.h.s. independently of $K$ and using monotone convergence gives the result. As an example, we discuss $R_{7}$, whose highest order part can be estimated by

$$
\begin{aligned}
\left|R_{7,2}\right| & \leq \delta \sigma \int_{\left[\rho_{i}>K\right]} \Psi^{n} K(\phi) K^{m_{2}+1-\frac{m_{1}+2}{2}} \rho_{i}^{m_{1} / 2}\left|\nabla \rho_{i}\right|^{2} \\
& +C_{\delta, \sigma}\left(\int_{\Omega_{T}} \Psi^{n}|\nabla V|^{\frac{5}{1-\varepsilon}}+\int_{\Omega_{T}} \Psi^{n} \rho_{i}^{\frac{5}{3+2 \varepsilon} m_{2}}\right)
\end{aligned}
$$

which implies the control condition $m_{2} \leq \frac{3+2 \varepsilon}{3}\left(m_{1}+2\right)$. Considering also the remaining terms, we get the control conditions

$$
\begin{aligned}
m_{2} & \leq \min \left(\frac{16}{15} m_{1}+\frac{17}{15}, \frac{4}{3} m_{1}+\frac{5}{3}, \frac{5}{3} m_{1}+\frac{4}{3}, \frac{3+2 \varepsilon}{3}\left(m_{1}+2\right), \frac{7}{6} m_{1}+\frac{4}{3}\right) \\
& =\min \left(\frac{3+2 \varepsilon}{3}\left(m_{1}+2\right), \frac{16}{15} m_{1}+\frac{17}{15}\right) .
\end{aligned}
$$

Copyright $@$ by SIAM. Unauthorized reproduction of this article is prohibited. 
The next result provides explicit $\sigma$-dependent local regularity of gradients of ion concentrations and will be used subsequently in the entropy estimates.

Lemma 3.8. Let $\Omega^{\prime} \subset \subset \Omega$ and $\left(\mathbf{v}, \rho_{0}, \rho_{1}, \rho_{2}, \phi, \mu, V\right)$ be a solution of (2.1). Then

$$
\sigma^{1 / 2} \rho_{1}, \sigma^{1 / 2} \rho_{2} \in L^{\infty}\left(I ; L^{2}\left(\Omega^{\prime}\right)\right) \cap L^{2}\left(I ; H^{1}\left(\Omega^{\prime}\right)\right)
$$

are bounded by a constant which depends only on dist $\left(\Omega^{\prime}, \partial \Omega\right)$ and the data, but not on the approximation parameters $\sigma, \sigma_{1}$, and $\Lambda_{1}$.

Proof. By the energy estimate (2.5),

$$
\int_{\Omega_{T}} K(\phi)\left[\frac{1}{M_{0}^{\prime \prime}\left(\rho_{1}\right)}\left|\nabla\left(V+M_{0}^{\prime}\left(\rho_{1}\right)\right)\right|^{2}+\frac{1}{M_{0}^{\prime \prime}\left(\rho_{2}\right)}\left|\nabla\left(-V+M_{0}^{\prime}\left(\rho_{2}\right)\right)\right|^{2}\right]<\infty .
$$

Take a cut-off function $\Psi \in C_{0}^{\infty}(\Omega ;[0,1])$ such that $\left.\Psi\right|_{\Omega^{\prime}} \equiv 1$. Hence, for $n \geq 3$

$$
\begin{gathered}
\infty>\int_{\Omega_{T}} \Psi^{n} K(\phi)\left[\frac{1}{M_{0}^{\prime \prime}\left(\rho_{1}\right)}|\nabla V|^{2}+\frac{1}{M_{0}^{\prime \prime}\left(\rho_{2}\right)}|\nabla V|^{2}+M_{0}^{\prime \prime}\left(\rho_{1}\right)\left|\nabla \rho_{1}\right|^{2}\right. \\
\left.+M_{0}^{\prime \prime}\left(\rho_{2}\right)\left|\nabla \rho_{2}\right|^{2}+2 \nabla V \cdot \nabla\left(\rho_{1}-\rho_{2}\right)\right] .
\end{gathered}
$$

By (2.1f),

$$
\begin{aligned}
& \int_{\Omega_{T}} \Psi^{n} K(\phi) \nabla V \cdot \nabla\left(\rho_{1}-\rho_{2}\right) \\
& =\int_{\Omega_{T}} \Psi^{n} \Gamma(\phi)\left(\rho_{1}-\rho_{2}\right)^{2}-\int_{\Omega_{T}} \Psi^{n} \varepsilon(\phi) \Gamma^{\prime}(\phi) \nabla \phi \cdot \nabla V\left(\rho_{1}-\rho_{2}\right) \\
& \quad-n \int_{\Omega_{T}} \Psi^{n-1} \nabla \Psi \cdot K(\phi) \nabla V\left(\rho_{1}-\rho_{2}\right) .
\end{aligned}
$$

Now estimate the second term on the r.h.s. by

$$
\begin{aligned}
& \left|\int_{\Omega_{T}} \Psi^{n} \varepsilon(\phi) \Gamma^{\prime}(\phi) \nabla \phi \cdot \nabla V\left(\rho_{1}-\rho_{2}\right)\right| \\
& \quad \leq \delta \int_{\Omega_{T}} \Psi^{n} \Gamma(\phi)\left(\rho_{1}-\rho_{2}\right)^{2}+C_{\delta}\left(\int_{\Omega_{T}}|\nabla \phi|^{6}+\int_{\Omega_{T}} \Psi^{n}|\nabla V|^{3}\right)
\end{aligned}
$$

and the last term on the r.h.s. by

$$
\left|n \int_{\Omega_{T}} \Psi^{n-1} \nabla \Psi \cdot K(\phi) \nabla V\left(\rho_{1}-\rho_{2}\right)\right| \leq \delta \int_{\Omega_{T}} \Psi^{n} \Gamma(\phi)\left(\rho_{1}-\rho_{2}\right)^{2}+C_{\delta} \int_{\Omega_{T}}|\nabla V|^{2},
$$

which gives the result.

Now, we are ready to prove higher regularity results for $\rho_{0}, \rho_{1}, \rho_{2}$, and $\nabla V$ which are independent of all the approximation parameters.

Lemma 3.9 ( $m$-entropy). Let $\left(\mathbf{v}, \rho_{0}, \rho_{1}, \rho_{2}, \phi, \mu, V\right)$ be a solution of (2.1). Then, for $i \in\{1,2\}$, any $m \in(0,1)$, and arbitrary $\Omega^{\prime} \subset \subset \Omega$, we have

$$
\rho_{i}^{(m+1) / 2} \in L^{\infty}\left(I ; L^{2}\left(\Omega^{\prime}\right)\right) \cap L^{2}\left(I ; H^{1}\left(\Omega^{\prime}\right)\right), \quad \rho_{i}^{\frac{5}{3}(m+1)} \in L^{1}\left(I ; L^{1}\left(\Omega^{\prime}\right)\right),
$$

where the constants depend only on $\operatorname{dist}\left(\Omega^{\prime}, \partial \Omega\right)$ and the data, but not on $\sigma, \sigma_{1}$, and $\Lambda_{1}$.

Copyright $@$ by SIAM. Unauthorized reproduction of this article is prohibited. 
Proof. We test in (2.1c) by $\psi_{2}=\Psi^{n}\left(\frac{1}{m} \rho_{i}^{m}+\frac{\sigma}{1+m} \rho_{i}^{m+1}\right)$ for $i \in\{1,2\}$ with $\Psi \in C_{0}^{\infty}(\Omega ;[0,1]),\left.\Psi\right|_{\Omega^{\prime}} \equiv 1, m \in(0,1), n \geq 6$. The admissibility of this test function is given by Lemma 3.7. Summing over $i \in\{1,2\}$, decomposing the elliptic terms, and putting in (2.1f) (cf. Lemma 3.2), we get

$$
\begin{aligned}
& \frac{1}{m(m+1)} \int_{\Omega} \Psi^{n}\left(\rho_{1}^{m+1}(T)+\rho_{2}^{m+1}(T)\right)+\frac{\sigma}{(m+1)(m+2)} \int_{\Omega} \Psi^{n}\left(\rho_{1}^{m+2}(T)+\rho_{2}^{m+2}(T)\right) \\
& +\int_{\Omega_{T}} \Psi^{n} K(\phi)\left[\rho_{1}^{m-1}\left|\nabla \rho_{1}\right|^{2}+\rho_{2}^{m-1}\left|\nabla \rho_{2}\right|^{2}+\sigma \rho_{1}^{m}\left|\nabla \rho_{1}\right|^{2}+\sigma \rho_{2}^{m}\left|\nabla \rho_{2}\right|^{2}\right] \\
& +\frac{1}{m} \int_{\Omega_{T}} \Psi^{n} K_{1}(\phi)\left(\rho_{1}^{m+1} \rho_{2}+\rho_{1} \rho_{2}^{m+1}\right)+\frac{\sigma}{1+m} \int_{\Omega_{T}} \Psi^{n} K_{1}(\phi)\left(\rho_{1}^{m+2} \rho_{2}+\rho_{1} \rho_{2}^{m+2}\right) \\
& +\frac{1}{m+1} \int_{\Omega_{T}} \Psi^{n} \Gamma(\phi)\left(\rho_{1}^{m+2}+\rho_{2}^{m+2}\right) \\
= & \frac{1}{m(m+1)} \int_{\Omega} \Psi^{n}\left(\rho_{1}^{m+1}(0)+\rho_{2}^{m+1}(0)\right)+\frac{\sigma}{(m+1)(m+2)} \int_{\Omega} \Psi^{n}\left(\rho_{1}^{m+2}(0)+\rho_{2}^{m+2}(0)\right) \\
& +\frac{1}{m} \int_{\Omega_{T}} \Psi^{n} K_{0}(\phi) \rho_{0}^{\alpha}\left(\rho_{1}^{m}+\rho_{2}^{m}\right)+\frac{\sigma}{m+1} \int_{\Omega_{T}} \Psi^{n} K_{0}(\phi) \rho_{0}^{\alpha}\left(\rho_{1}^{m+1}+\rho_{2}^{m+1}\right) \\
& -\int_{\Omega_{T}} \Psi^{n} \mathbf{v} \cdot\left[\nabla\left(\frac{\rho_{1}}{1+\sigma \rho_{1}}\right)\left(\frac{1}{m} \rho_{1}^{m}+\frac{\sigma}{m+1} \rho_{1}^{m+1}\right)+\nabla\left(\frac{\rho_{2}}{1+\sigma \rho_{2}}\right)\left(\frac{1}{m} \rho_{2}^{m}+\frac{\sigma}{m+1} \rho_{2}^{m+1}\right)\right] \\
& +\frac{1}{m+1} \int_{\Omega_{T}} \Psi^{n} \Gamma^{\prime}(\phi) \nabla \phi \cdot \varepsilon(\phi) \nabla V\left(\rho_{1}^{m+1}-\rho_{2}^{m+1}\right) \\
& +\frac{1}{m+1} \int_{\Omega_{T}} \Psi^{n} \Gamma(\phi)\left(\rho_{1}^{m+1} \rho_{2}+\rho_{1} \rho_{2}^{m+1}\right) \\
& -\frac{n}{m+1} \int_{\Omega_{T}} \Psi^{n-1} K(\phi) \nabla \Psi \cdot\left[\rho_{1}^{m} \nabla \rho_{1}+\rho_{2}^{m} \nabla \rho_{2}+\sigma \rho_{1}^{m+1} \nabla \rho_{1}+\sigma \rho_{2}^{m+1} \nabla \rho_{2}\right] \\
& -\frac{n}{m(m+1)} \int_{\Omega_{T}} \Psi^{n-1} K(\phi) \nabla \Psi \cdot \nabla V\left(\frac{\rho_{1}^{m+1}}{1+\sigma \rho_{1}}-\frac{\rho_{2}^{m+1}}{1+\sigma \rho_{2}}\right) \\
& -\frac{n}{m(m+1)} \int_{\Omega_{T}} \Psi^{n-1} K(\phi) \nabla \Psi \cdot\left(\rho_{1}^{m} \nabla \rho_{1}+\rho_{2}^{m} \nabla \rho_{2}\right) \\
= & R_{1}+\cdots+R_{10} .
\end{aligned}
$$

Here, we used again that $\frac{1}{m} \rho_{i}^{m}+\frac{\sigma}{1+m} \rho_{i}^{m+1}=\frac{\rho_{i}^{m}}{m+1}\left(1+\sigma \rho_{i}\right)+\frac{1}{m(m+1)} \rho_{i}^{m}$. Obviously, we find $\left|R_{1}\right|+\left|R_{2}\right| \leq C$. For $i \in\{1,2\}$ we have with $p<\frac{3(m+1)}{m}$ such that $p^{\prime}>\frac{3(m+1)}{2 m+3}$

$$
\begin{aligned}
\left|R_{3}\right| & \leq C \int_{\Omega_{T}} \rho_{0}^{\alpha} \Psi^{n} \rho_{i}^{m} \leq C \int_{\Omega_{T}} \rho_{0}^{\alpha}\left(\Psi^{\frac{n}{2}} \rho_{i}^{\frac{m+1}{2}}\right)^{\frac{2 m}{m+1}} \\
& \leq C\left(\int_{0}^{T}\left(\int_{\Omega} \rho_{0}^{\alpha p^{\prime}}\right)^{\frac{m+1}{p^{\prime}}}\right)^{\frac{1}{m+1}}\left(\int_{0}^{T}\left(\int_{\Omega}\left(\Psi^{\frac{n}{2}} \rho_{i}^{\frac{m+1}{2}}\right)^{\frac{2 m p}{m+1}}\right)^{\frac{m+1}{p m}}\right)^{\frac{m}{m+1}} .
\end{aligned}
$$

The first integral on the r.h.s. is bounded if $\rho_{0}^{\alpha / 2} \in L^{2(m+1)}\left(I ; L^{2 p^{\prime}}(\Omega)\right)$. For $\alpha \in\left(0, \frac{1}{2}\right]$, this follows from (3.1). For $\alpha \in\left(\frac{1}{2}, 1\right)$, we infer from Lemma 3.1 that, independent of approximation parameters, $\rho_{0}^{\alpha / 2} \in L^{\infty}\left(I ; L^{2}(\Omega)\right) \cap L^{2}\left(I ; H^{1}(\Omega)\right) \hookrightarrow L^{r}\left(I ; L^{q}(\Omega)\right)$ with $\frac{1}{r}+\frac{3}{2 q}=\frac{3}{4}$ (see Lemma C.2). Inserting $r=2(m+1)$ and $q=2 p^{\prime}$ yields $m<2$.

Copyright $@$ by SIAM. Unauthorized reproduction of this article is prohibited. 
Thus,

$$
\left|R_{3}\right| \leq \int_{0}^{T}\left\|\Psi^{\frac{n}{2}} \rho_{i}^{\frac{m+1}{2}}\right\|_{L^{2 m p /(m+1)}(\Omega)}^{2}+C
$$

Since $\frac{2 m p}{m+1}<6$, Lemma C.4 implies

$$
\left|R_{3}\right| \leq \delta \int_{\Omega_{T}} \Psi^{n} \rho_{i}^{m-1}\left|\nabla \rho_{i}\right|^{2}+C_{\delta}\left(\int_{\Omega_{T}} \Psi^{n-2} \rho_{i}^{m+1}+\int_{\Omega_{T}} \Psi^{n} \rho_{i}^{m+1}\right)+C .
$$

The $\delta$-term can be absorbed. The most critical rest term can be estimated by

$$
C_{\delta} \int_{\Omega_{T}} \Psi^{n-2} \rho_{i}^{m+1} \leq \bar{\delta} \int_{\Omega_{T}} \Psi^{(n-2) \frac{m+2}{m+1}} \rho_{i}^{m+2}+C_{\delta, \bar{\delta}}
$$

Now, the $\bar{\delta}$-term can be absorbed into the $\Gamma$-term on the l.h.s. of (3.6), provided $n \geq 4+2 m$. This means $n \geq 6$, which we had assumed. Similarly, we estimate with $p<\frac{3(m+2)}{m+1}$ and thus $p^{\prime}>\frac{3(m+2)}{2 m+5}$

$$
\begin{aligned}
\left|R_{4}\right| & \leq \sigma C \int_{\Omega_{T}} \rho_{0}^{\alpha} \Psi^{n} \rho_{i}^{m+1} \leq \sigma C \int_{\Omega_{T}} \rho_{0}^{\alpha}\left(\Psi^{\frac{n}{2}} \rho_{i}^{\frac{m+2}{2}}\right)^{\frac{2(m+1)}{m+2}} \\
& \leq \sigma C\left(\int_{0}^{T}\left(\int_{\Omega} \rho_{0}^{\alpha p^{\prime}}\right)^{\frac{m+2}{p^{\prime}}}\right)^{\frac{1}{m+2}}\left(\int_{0}^{T}\left(\int_{\Omega}\left(\Psi^{\frac{n}{2}} \rho_{i}^{\frac{m+2}{2}}\right)^{\frac{2(m+1) p}{m+2}}\right)^{\frac{m+2}{p(m+1)}}\right)^{\frac{m+1}{m+2}} .
\end{aligned}
$$

As for $R_{3}$, the first integral on the r.h.s. is bounded if $m<1$. Thus, we can further estimate

$$
\left|R_{4}\right| \leq \sigma^{\frac{m+2}{m+1}} \int_{0}^{T}\left\|\Psi^{\frac{n}{2}} \rho_{i}^{\frac{m+2}{2}}\right\|_{L^{\frac{2(m+1) p}{m+2}}(\Omega)}^{2}+C
$$

By Lemma C.4,

$$
\left|R_{4}\right| \leq \delta \sigma \int_{\Omega_{T}} \Psi^{n} \rho_{i}^{m}\left|\nabla \rho_{i}\right|^{2}+C_{\delta} \sigma^{\frac{m+2}{m+1}}\left(\int_{\Omega_{T}} \Psi^{n-2} \rho_{i}^{m+2}+\int_{\Omega_{T}} \Psi^{n} \rho_{i}^{m+2}\right)+C .
$$

The $\delta$-term can be absorbed. For the most critical remaining term, we find

$$
C_{\delta} \sigma^{\frac{m+2}{m+1}} \int_{\Omega_{T}} \Psi^{n-2} \rho_{i}^{m+2} \leq C_{\delta} \sigma^{\frac{m+2}{m+1}-\frac{m+2}{2}} \int_{[\operatorname{supp} \Psi \times I]}\left(\sigma^{\frac{1}{2}} \rho_{i}\right)^{m+2} \leq C .
$$

This bound holds if $m \leq 1$ and $m \leq \frac{4}{3}$ are fulfilled. The term $\left|R_{5}\right|$ can be estimated similarly to the corresponding term $\left|R_{5}\right|$ in Lemma 3.2, giving as before the bound $m \leq \frac{4}{3}$. Using $\nabla V \in L^{\infty}\left(I ; L^{2}(\Omega)\right), \nabla \phi \in L^{\infty}\left(I ; L^{s}(\Omega)\right)$, and Lemma C.4, we estimate with $p=\frac{2 s}{s-2}<3$ (remember $s>6$ )

$$
\begin{aligned}
\left|R_{6}\right| & \leq C \int_{0}^{T}\left(\int_{\Omega}|\nabla V|^{2}\right)^{1 / 2}\left(\int_{\Omega}|\nabla \phi|^{s}\right)^{1 / s}\left(\int_{\Omega}\left(\Psi^{n} \rho_{i}^{m+1}\right)^{p}\right)^{1 / p} \\
& \leq C \int_{0}^{T}\left(\int_{\Omega}\left(\Psi^{n} \rho_{i}^{m+1}\right)^{p}\right)^{1 / p}=C \int_{0}^{T}\left(\int_{\Omega}\left(\Psi^{\frac{n}{2}} \rho_{i}^{\frac{m+1}{2}}\right)^{2 p}\right)^{2 / 2 p} \\
& \leq \delta \int_{\Omega_{T}} \Psi^{n} \rho_{i}^{m-1}\left|\nabla \rho_{i}\right|^{2}+C_{\delta}\left(\int_{\Omega_{T}} \Psi^{n-2} \rho_{i}^{m+1}+\int_{\Omega_{T}} \Psi^{n} \rho_{i}^{m+1}\right) .
\end{aligned}
$$

Copyright $($ C by SIAM. Unauthorized reproduction of this article is prohibited. 
The $\delta$-term can be absorbed on the l.h.s. of (3.6), and the other two terms can be treated as in $R_{3}$ above. $R_{7}$ can be estimated by the fourth term on the l.h.s. due to assumption $\left(A_{2}\right)$. Further, $R_{8.1}$ and $R_{10}$ can be estimated by

$$
\begin{aligned}
\left|R_{8.1}\right|+\left|R_{10}\right| & \leq \delta_{1} \int_{\Omega_{T}} \Psi^{n} K(\phi) \rho_{i}^{m-1}\left|\nabla \rho_{i}\right|^{2}+\delta_{2} \int_{\Omega_{T}} \Psi^{n} \Gamma(\phi) \rho_{i}^{m+2} \\
& +C_{\Gamma, \delta_{1}, \delta_{2}} \int_{\Omega_{T}} \Psi^{n-2(2+m)} .
\end{aligned}
$$

Here, $n \geq 6$ is needed again for the last term on the r.h.s. By a generalized Young's inequality, we have

$$
\begin{aligned}
& \left|R_{8.2}\right|=\left|\sigma \int_{\Omega_{T}} \Psi^{n-1} K(\phi) \rho_{i}^{m+1} \nabla \rho_{i} \cdot \nabla \Psi\right| \\
& \quad \leq C_{\delta, \Gamma, K} \int_{[\operatorname{supp} \Psi] \times I} \sigma^{\frac{5}{3}} \rho_{i}^{\frac{10}{3}}+\delta\left\{\sigma \int_{\Omega_{T}} \Psi^{n} K(\phi) \rho_{i}^{m}\left|\nabla \rho_{i}\right|^{2}+\int_{\Omega_{T}} \Psi^{\frac{5 n-10}{2}} \Gamma(\Phi) \rho_{i}^{\frac{5 m}{2}}\right\} .
\end{aligned}
$$

The last two terms can be absorbed on the l.h.s., provided $n>10 / 3$ and $m \leq 4 / 3$. The first term is controlled by a combination of Lemma 3.8, the $L^{\infty}\left(L^{2}\right)$-bound of $\sigma^{1 / 2} \rho_{i}$, and Lemma C.1. Finally,

$$
\left|R_{9}\right| \leq \delta \int_{\Omega_{T}} \Psi^{n} \Gamma(\phi) \rho_{i}^{m+2}+C_{\delta, \Psi} \int_{\Omega_{T}} \Psi^{n-2-m}|\nabla V|^{2+m},
$$

and the result follows by absorption and the $L^{3}\left(L_{\text {loc }}^{3}\right)$-bound on $\nabla V$.

Enhanced regularity of $\rho_{0}$ comes as a direct consequence.

LEMma 3.10 (regularity for $\rho_{0}(\mathrm{II})$ ). Let $\Omega^{\prime} \subset \subset \Omega$ be arbitrary, $\rho_{i} \in L^{p}\left(I ; L^{p}\left(\Omega^{\prime}\right)\right)$ for $i \in\{1,2\}, p>2$, and $\rho_{0} \in L^{r}\left(I ; L^{r}\left(\Omega^{\prime}\right)\right)$ for $r>\frac{10}{7}$. Then we have

$$
\rho_{0}^{\min \left(\frac{p}{4}, \frac{7}{20} r\right)} \in L^{\infty}\left(I ; L^{2}\left(\Omega^{\prime}\right)\right) \cap L^{2}\left(I ; H^{1}\left(\Omega^{\prime}\right)\right)
$$

and

$$
\rho_{0}^{\min }\left(\frac{5}{6} p, \frac{7}{6} r\right) \in L^{1}\left(I ; L^{1}\left(\Omega^{\prime}\right)\right)
$$

where the constants depend on $\operatorname{dist}\left(\Omega^{\prime}, \partial \Omega\right)$ and the data, but not on $\sigma, \sigma_{1}$, and $\Lambda_{1}$.

Proof. The second estimate follows immediately by Lemma C.1. To prove the first estimate, test $(2.1 \mathrm{~b})$ by $\psi_{1}:=\frac{1}{\beta} \Psi^{n} \rho_{0}^{\beta}, \beta>0, \Psi \in C_{0}^{\infty}(\Omega ;[0,1]),\left.\Psi\right|_{\Omega^{\prime}} \equiv 1, n \geq 4$ (the admissibility is given by Lemma 3.5 ), to get

$$
\begin{aligned}
\frac{1}{\beta(\beta+1)} & \int_{\Omega} \Psi^{n} \rho_{0}^{\beta+1}(T)+\int_{\Omega_{T}} \Psi^{n} K(\phi) \rho_{0}^{\beta-1}\left|\nabla \rho_{0}\right|^{2}+\frac{1}{\beta} \int_{\Omega_{T}} \Psi^{n} K_{0}(\phi) \rho_{0}^{\alpha+\beta} \\
= & \frac{1}{\beta(\beta+1)} \int_{\Omega} \Psi^{n} \rho_{0}^{\beta+1}(0)+\frac{1}{\beta} \int_{\Omega_{T}} \Psi^{n} K_{1}(\phi) \bar{M}_{\Lambda_{1}}\left(\rho_{1}\right) \bar{M}_{\Lambda_{1}}\left(\rho_{2}\right) \rho_{0}^{\beta} \\
& \quad-\frac{n}{\beta} \int_{\Omega_{T}} \Psi^{n-1} K(\phi) \rho_{0}^{\beta} \nabla \Psi \cdot \nabla \rho_{0}+\frac{n}{\beta(\beta+1)} \int_{\Omega_{T}} \Psi^{n-1} \mathbf{v} \cdot \nabla \Psi \rho_{0}^{\beta+1} \\
= & R_{1}+\cdots+R_{4} .
\end{aligned}
$$

Obviously, $\left|R_{1}\right| \leq C$, due to the local boundedness of initial data. Moreover,

$$
\left|R_{2}\right| \leq C \int_{\Omega_{T}} \Psi^{n}\left(\rho_{1} \rho_{2}\right)^{p / 2}+C \int_{\Omega_{T}} \Psi^{n} \rho_{0}^{\beta \frac{p}{p-2}}
$$

Copyright (c) by SIAM. Unauthorized reproduction of this article is prohibited. 
which can be controlled by the assumptions of the lemma combined with Gronwall's inequality, provided $\beta+1 \leq \frac{p}{2}$. The third term on the r.h.s. is readily estimated by

$$
\left|R_{3}\right| \leq \delta \int_{\Omega_{T}} \Psi^{n} K(\phi) \rho_{0}^{\beta-1}\left|\nabla \rho_{0}\right|^{2}+C_{\delta} \int_{\Omega_{T}} \Psi^{n-2} K(\phi) \rho_{0}^{\beta+1},
$$

and the last term can be controlled if $\beta+1 \leq r$. Finally,

$$
\left|R_{4}\right| \leq \delta \int_{\Omega_{T}} \Psi^{\frac{10}{7}(n-1)} \rho_{0}^{\frac{10}{7}(\beta+1)}+C_{\delta} \int_{\Omega_{T}}|\mathbf{v}|^{10 / 3},
$$

and the first term on the r.h.s. can be controlled by the $L^{r}\left(I ; L^{r}\left(\Omega^{\prime}\right)\right)$-regularity as long as $\beta+1 \leq \frac{7 r}{10}$.

COROLLARY 3.11. Under the assumptions of Lemma 3.10,

$$
\rho_{0}^{\frac{p}{4}} \in L^{\infty}\left(I ; L^{2}\left(\Omega^{\prime}\right)\right) \cap L^{2}\left(I ; H^{1}\left(\Omega^{\prime}\right)\right)
$$

and

$$
\rho_{0}^{\frac{5}{6} p} \in L^{1}\left(I ; L^{1}\left(\Omega^{\prime}\right)\right)
$$

with bounds depending only on dist $\left(\partial \Omega, \Omega^{\prime}\right)$, but independent of $\sigma, \sigma_{1}$, and $\Lambda_{1}$.

Proof. By Lemma 3.10,

$$
\rho_{0}^{\min \left(\frac{p}{4}, \frac{7}{20} r\right)} \in L^{\infty}\left(I ; L^{2}\left(\Omega^{\prime}\right)\right) \cap L^{2}\left(I ; H^{1}\left(\Omega^{\prime}\right)\right),
$$

and by Lemma C.1,

$$
\rho_{0}^{\min \left(\frac{5}{6} p, \frac{7}{6} r\right)} \in L^{1}\left(I ; L^{1}\left(\Omega^{\prime}\right)\right) .
$$

If $\frac{7}{6} r<\frac{5}{6} p$, apply Lemma 3.10 iteratively until $\frac{7}{6} r \geq \frac{5}{6} p$. Obviously, the terminal condition will be reached with finitely many steps.

Combining Lemmas 3.1, 3.9, and 3.10 and Corollaries 3.11 and 3.4 yields the following.

Corollary 3.12. For any $\Omega^{\prime} \subset \subset \Omega$ it holds that

$$
\begin{aligned}
& \rho_{0}^{p} \in L^{\infty}\left(I ; L^{2}\left(\Omega^{\prime}\right)\right) \cap L^{2}\left(I ; H^{1}\left(\Omega^{\prime}\right)\right) \text { for any } p \in\left(\frac{1-\alpha}{2}, \frac{5}{6}\right), \\
& \rho_{0}^{p} \in L^{1}\left(I ; L^{1}\left(\Omega^{\prime}\right)\right) \text { for any } p \in\left(0, \frac{25}{9}\right), \text { and } \\
& |\nabla V|^{p} \in L^{1}\left(I ; L^{1}\left(\Omega^{\prime}\right)\right) \text { for any } p \in\left(0, \frac{16}{3}\right)
\end{aligned}
$$

independent of $\sigma, \sigma_{1}$, and $\Lambda_{1}$.

We now formulate the main iteration lemma.

Lemma 3.13 (iteration). Let $\Omega^{\prime} \subset \subset \Omega$ be arbitrary, let

$$
\rho_{0}^{p} \in L^{\infty}\left(I ; L^{2}\left(\Omega^{\prime}\right)\right) \cap L^{2}\left(I ; H^{1}\left(\Omega^{\prime}\right)\right)
$$

for any $p \in\left(\frac{1-\alpha}{2}, \frac{5}{6}\right)$, and let $m_{1}>\frac{1}{2}$ such that

$$
\rho_{i}^{\left(m_{1}+1\right) / 2}, \sigma^{1 / 2} \rho_{i}^{\left(m_{1}+2\right) / 2} \in L^{\infty}\left(I ; L^{2}\left(\Omega^{\prime}\right)\right) \cap L^{2}\left(I ; H^{1}\left(\Omega^{\prime}\right)\right)
$$

Copyright $@$ by SIAM. Unauthorized reproduction of this article is prohibited. 
for $i \in\{1,2\}$. Then, for any $m_{2} \leq \min \left(m_{1}+1, \frac{7}{6} m_{1}+\frac{1}{6}, \frac{5}{3} m_{1}-\frac{1}{3}\right)$,

$$
\rho_{i}^{\left(m_{2}+1\right) / 2}, \sigma^{1 / 2} \rho_{i}^{\left(m_{2}+2\right) / 2} \in L^{\infty}\left(I ; L^{2}\left(\Omega^{\prime}\right)\right) \cap L^{2}\left(I ; H^{1}\left(\Omega^{\prime}\right)\right) \text { for } i \in\{1,2\},
$$

independent of all the approximation parameters. By iteration, one can achieve any $m_{2}<\infty$ with finitely many steps.

Proof. The starting point is a localized $m_{2}$-entropy estimate analogous to (3.6). For $\Psi \in C_{0}^{\infty}(\Omega,[0,1]),\left.\Psi\right|_{\Omega^{\prime}} \equiv 1, n \geq 4+2 m_{2}$, we use the test function

$$
\psi_{2}=\Psi^{n}\left(\frac{1}{m_{2}} \rho_{i}^{m_{2}}+\frac{\sigma}{1+m_{2}} \rho_{i}^{m_{2}+1}\right)
$$

in (2.1c), which is admissible due to Lemma 3.7. As in Lemma 3.9, we get (3.6) with $m$ replaced by $m_{2}$, and thus inheriting the structure

$$
\text { l.h.s. } \leq \text { r.h.s. }=R_{1}+\cdots+R_{10} \text {. }
$$

Estimating the r.h.s., we start with $\left|R_{1}\right|+\left|R_{2}\right| \leq C$. By Lemma C.4 and (3.9),

$$
\begin{aligned}
\left|R_{3}\right| & \leq C \int_{0}^{T}\left(\int_{\Omega} \Psi^{\frac{5 n}{3\left(m_{2}+1\right)}} \rho_{0}^{\frac{5}{3} \alpha}\right)^{\frac{3}{5}}\left(\int_{\Omega}\left(\Psi^{\frac{n}{2}} \rho_{i}^{\frac{m_{2}+1}{2}}\right)^{\frac{5 m_{2}}{m_{2}+1}}\right)^{\frac{2}{5}} \\
& \leq \delta \int_{\Omega_{T}} \Psi^{n} \rho_{i}^{m_{2}-1}\left|\nabla \rho_{i}\right|^{2}+C_{\delta}\left(\int_{\Omega_{T}} \Psi^{n-2} \rho_{i}^{m_{2}+1}+\int_{\Omega_{T}} \Psi^{n} \rho_{i}^{m_{2}+1}\right)+C .
\end{aligned}
$$

Absorbing the $\delta$-term and estimating the most critical term gives

$$
C_{\delta} \int_{\Omega_{T}} \Psi^{n-2} \rho_{i}^{m_{2}+1} \leq \bar{\delta} \int_{\Omega_{T}} \Psi^{(n-2) \frac{m_{2}+2}{m_{2}+1}} \rho_{i}^{m_{2}+2}+C_{\delta, \bar{\delta}}
$$

The $\bar{\delta}$-term can be absorbed, provided $n \geq 4+2 m_{2}$. Similarly,

$$
\begin{aligned}
\left|R_{4}\right| & \leq C \sigma \int_{0}^{T}\left(\int_{\Omega} \Psi^{\frac{5 n}{3\left(m_{2}+2\right)}} \rho_{0}^{\frac{5}{3} \alpha}\right)^{\frac{3}{5}}\left(\int_{\Omega}\left(\Psi^{\frac{n}{2}} \rho_{i}^{\frac{m_{2}+2}{2}}\right)^{\frac{5\left(m_{2}+1\right)}{m_{2}+2}}\right)^{\frac{2}{5}} \\
& \leq C \sigma \int_{0}^{T}\left\|\Psi^{\frac{n}{2}} \rho_{i}^{\frac{m_{2}+2}{2}}\right\|_{L^{\frac{5\left(m_{2}+1\right)}{m_{2}+2}}}^{2 \frac{m_{2}+1}{m_{2}+2}}(\Omega) \\
& \leq \delta \sigma \int_{\Omega_{T}} \Psi^{n} \rho_{i}^{m_{2}}\left|\nabla \rho_{i}\right|^{2}+C_{\delta} \sigma^{\frac{m_{2}+2}{m_{2}+1}}\left(\int_{\Omega_{T}} \Psi^{n-2} \rho_{i}^{m_{2}+2}+\int_{\Omega_{T}} \Psi^{n} \rho_{i}^{m_{2}+2}\right)+C .
\end{aligned}
$$

Proceeding as before, we estimate

$$
\begin{aligned}
C_{\delta} \sigma^{\frac{m_{2}+2}{m_{2}+1}} \int_{\Omega_{T}} \Psi^{n-2} \rho_{i}^{m_{2}+2} & =C_{\delta} \int_{\Omega_{T}}\left(\Psi^{\frac{n-2}{m_{2}+2}} \sigma^{\frac{1}{m_{2}+1}-\frac{1}{m_{1}+2}} \sigma^{\frac{1}{m_{1}+2}} \rho_{i}\right)^{m_{2}+2} \\
& \leq C_{\delta} \int_{[\operatorname{supp} \Psi \times I]}\left(\sigma^{\frac{1}{m_{1}+2}} \rho_{i}\right)^{m_{2}+2}
\end{aligned}
$$

provided $m_{2} \leq m_{1}+1$. Note that $\sigma^{1 / 2} \rho_{i}^{\left(m_{1}+2\right) / 2} \in L^{\infty}\left(I ; L^{2}\left(\Omega^{\prime}\right)\right) \cap L^{2}\left(I ; H^{1}\left(\Omega^{\prime}\right)\right)$ and that Lemma C.3 implies $\sigma^{\frac{1}{m_{1}+2}} \rho_{i} \in L^{\frac{5}{3}\left(m_{1}+2\right)}\left(\Omega_{T}^{\prime}\right)$, which gives the second condition, $m_{2} \leq \frac{5}{3} m_{1}+\frac{4}{3}$. The term $R_{5}$ can be estimated as in Lemma 3.2 , which yields 
the absorption condition $m_{2} \leq \frac{1}{6}\left(7 m_{1}+1\right)$. The term $R_{6}$ can be estimated as the corresponding term in Lemma 3.9, giving no further condition for $m_{2}$, but the power $n$ of $\Psi$ has to be chosen such that $n \geq 4+2 m_{2} . R_{7}$ is again controlled by assumption $\left(A_{2}\right)$, implying no other condition for $m_{2}$. Further on,

$$
\left|R_{8.1}\right|+\left|R_{10}\right| \leq \delta \int_{\Omega_{T}} \Psi^{n} K(\phi) \rho_{i}^{m_{2}-1}\left|\nabla \rho_{i}\right|^{2}+C_{\delta, \Psi} \int_{\Omega_{T}} \Psi^{n-2} \rho_{i}^{m_{2}+1} .
$$

Absorb the $\delta$-term on the l.h.s. The second term on the r.h.s. can be estimated as the corresponding term in $R_{3}$, giving no condition for $m_{2}$, but only the already known condition for $n$. We continue with

$$
\left|R_{8.2}\right| \leq \delta \sigma^{2} \int_{\Omega_{T}} \Psi^{n} K(\phi) \rho_{i}^{m_{2}}\left|\nabla \rho_{i}\right|^{2}+C_{\delta} \int_{\Omega_{T}} \Psi^{n-2} \rho_{i}^{m_{2}+2},
$$

and hence $m_{2} \leq \frac{5}{3} m_{1}-\frac{1}{3}$ is required. Note that $\frac{5}{3} m_{1}-\frac{1}{3}>m_{1}$ iff $m_{1}>\frac{1}{2}$. Finally, $R_{9}$ has better integrability properties than $R_{6}$ due to the boundedness of $\left(1+\sigma \rho_{i}\right)^{-1}$. Hence, estimating this term does not give rise to further conditions on $m_{2}$. Collecting all bounds on $m_{2}$, we get

$$
\begin{aligned}
m_{2} & :=\min \left(m_{1}+1, \frac{5}{3} m_{1}+\frac{4}{3}, \frac{7}{6} m_{1}+\frac{1}{6}, \frac{5}{3} m_{1}-\frac{1}{3}\right) \\
& =\min \left(m_{1}+1, \frac{7}{6} m_{1}+\frac{1}{6}, \frac{5}{3} m_{1}-\frac{1}{3}\right) .
\end{aligned}
$$

As a straightforward consequence of Lemma 3.13, we obtain the following.

COROLLARY 3.14. Let $\Omega^{\prime} \subset \subset \Omega$ be arbitrary. It holds that

$$
\rho_{0}^{p} \in L^{\infty}\left(I ; L^{2}\left(\Omega^{\prime}\right)\right) \cap L^{2}\left(I ; H^{1}\left(\Omega^{\prime}\right)\right)
$$

for any $p<\infty$ independent of all approximation parameters.

Proof of Theorem 1.1. In particular, we get from this iteration procedure, independent of any approximation parameters,

$$
\rho_{i} \in L^{\infty}\left(I ; L^{2}\left(\Omega^{\prime}\right)\right) \cap L^{2}\left(I ; H^{1}\left(\Omega^{\prime}\right)\right)
$$

for $i \in\{0,1,2\}$ and any $\Omega^{\prime} \subset \subset \Omega$. Using this regularity and the approximation parameter-independent bounds from the VEMR energy estimate (2.5), we now show compactness in time independently of any approximation parameters. Let $\Omega^{\prime} \subset \subset \Omega$ be arbitrary, but fixed. Choose $\psi_{1} \in L^{5}\left(I ; H_{0}^{1}\left(\Omega^{\prime}\right)\right.$ ) (with its trivial extension to $\left.\psi_{1} \in L^{5}\left(I ; H_{0}^{1}(\Omega)\right)\right)$ as test function in $(2.1 \mathrm{~b})$ and estimate

$$
\begin{aligned}
&\left|\int_{0}^{T}\left\langle\rho_{0, t}, \psi_{1}\right\rangle\right| \leq\left|\int_{\Omega_{T}^{\prime}} K(\phi) \nabla \rho_{0} \cdot \nabla \psi_{1}\right|+\left|\int_{\Omega_{T}^{\prime}} \rho_{0} \mathbf{v} \cdot \nabla \psi_{1}\right| \\
& \quad+\left|\int_{\Omega_{T}^{\prime}} K_{0}(\phi) \rho_{0}^{\alpha} \psi_{1}\right|+\left|\int_{\Omega_{T}^{\prime}} K_{1}(\phi) \bar{M}_{\Lambda_{1}}\left(\rho_{1}\right) \bar{M}_{\Lambda_{1}}\left(\rho_{2}\right) \psi_{1}\right| \\
& \leq C\left\|\nabla \rho_{0}\right\|_{L^{2}\left(\Omega_{T}^{\prime}\right)}\left\|\nabla \psi_{1}\right\|_{L^{2}\left(\Omega_{T}^{\prime}\right)}+\|\mathbf{v}\|_{L^{10 / 3}\left(\Omega_{T}\right)}\left\|\nabla \rho_{0}\right\|_{L^{2}\left(\Omega_{T}^{\prime}\right)}\left\|\psi_{1}\right\|_{L^{5}\left(\Omega_{T}^{\prime}\right)} \\
&+C\left\|\rho_{0}\right\|_{L^{2}\left(\Omega_{T}^{\prime}\right)}\left\|\psi_{1}\right\|_{L^{2}\left(\Omega_{T}^{\prime}\right)}+C\left\|\rho_{1}\right\|_{L^{3}\left(\Omega_{T}^{\prime}\right)}\left\|\rho_{2}\right\|_{L^{3}\left(\Omega_{T}^{\prime}\right)}\left\|\psi_{1}\right\|_{L^{3}\left(\Omega_{T}^{\prime}\right)} .
\end{aligned}
$$

This means $\rho_{0} \in W^{1,5 / 4}\left(I ;\left(H_{0}^{1}\left(\Omega^{\prime}\right)\right)^{\prime}\right)$. Analogously,

$$
\rho_{i} \in W^{1,3 / 2}\left(I ;\left(W_{0}^{1,3}\left(\Omega^{\prime}\right)\right)^{\prime}\right) \text { for } i \in\{1,2\}, \quad \mathbf{v} \in W^{1,6 / 5}\left(I ;\left(H_{0}^{1}\left(\Omega^{\prime}\right)\right)^{\prime}\right),
$$

Copyright $@$ by SIAM. Unauthorized reproduction of this article is prohibited. 
and as before $\phi \in H^{1}\left(I ;\left(H^{1}(\Omega)\right)^{\prime}\right)$. With the compactness theorems of Simon (see [27, Cor. 4, p. 85]) and of Aubin and Lions, we get strong convergence of $\mathbf{v}, \phi, \rho_{i}$, $i \in\{0,1,2\}$, in the respective spaces. As in Eck et al. [11, p. 278], we can show the strong convergence of $V$ in $L^{2}\left(I ; H^{1}\left(\Omega^{\prime}\right)\right)$. Now, we pass to the limit $\left(\sigma, \sigma_{1}, \Lambda_{1}\right) \rightarrow$ $(0,0, \infty)$ in $(2.1)$ to get equations $(1.5)$. Note that the convergence in $(2.1 \mathrm{e})$ follows again similarly to the convergence of (B.2e) at the end of Appendix B using the pseudomonotonicity of $-\Delta_{s}$.

Finally, we prove conservation of total species mass. On the VEMR level, we obtain by testing $(2.1 \mathrm{~b})$ and $(2.1 \mathrm{c})$ with $\psi_{1}=\psi_{2} \equiv 1$ and summation that

$$
\left[\int_{\Omega} 2 \rho_{0}^{\mathbf{s}}+\rho_{1}^{\mathbf{s}}+\rho_{2}^{\mathbf{s}}\right]_{0}^{T}+\int_{\Omega_{T}} 2 K_{1}\left(\phi^{\mathbf{s}}\right)\left(\rho_{1}^{\mathbf{s}} \rho_{2}^{\mathbf{s}}-\bar{M}_{\Lambda_{1}}\left(\rho_{1}^{\mathbf{s}}\right) \bar{M}_{\Lambda_{1}}\left(\rho_{2}^{\mathbf{s}}\right)\right)=0
$$

with $\mathbf{s}=\left(\sigma, \sigma_{1}, \Lambda_{1}\right)$. Hence, it will be sufficient to show that the second term on the l.h.s. tends to zero for each $T>0$ in the limit $\mathbf{s} \rightarrow(0,0, \infty)$. By a diagonal argument and the convergences established so far, we infer the existence of a subsequence $\mathbf{s} \rightarrow(0,0, \infty)$ such that $\rho_{1}^{\mathbf{s}}, \rho_{2}^{\mathbf{s}}$ converge pointwise a.e. on $\Omega_{T}$ to $\rho_{1}, \rho_{2}$, respectively. Combining the uniform boundedness of $\int_{\left[\min \left(\rho_{1}^{\mathrm{s}}, \rho_{2}^{\mathrm{s}}\right) \geq 1\right]} K_{1}\left(\phi^{\mathbf{s}}\right) \rho_{1}^{\mathbf{s}} \rho_{2}^{\mathbf{s}} \log \left(\rho_{1}^{\mathbf{s}} \rho_{2}^{\mathbf{s}}\right)$ with Vitali's convergence theorem, we infer the $L^{1}$-convergence of $\rho_{1}^{\mathbf{s}} \rho_{2}^{\mathbf{s}}$ towards $\rho_{1} \rho_{2}$. Obviously, the same holds for the product $\bar{M}_{\Lambda_{1}}\left(\rho_{1}^{\mathbf{s}}\right) \bar{M}_{\Lambda_{1}}\left(\rho_{2}^{\mathrm{s}}\right)$. Hence, in the limit the second term on the r.h.s. vanishes. From this, the result follows in a standard way.

Remark 3.15. If sufficiently smooth, solutions as constructed in the proof of Theorem 1.1 satisfy the no-flux boundary conditions (1.2). Let us sketch the argument. For the ion densities, the starting point is the weak formulation (2.1c) in the VEMR model. Introducing the flux $\mathcal{J}_{i}^{\mathbf{s}}:=K\left(\phi^{\mathbf{s}}\right) \frac{1}{M_{0}^{\prime \prime}\left(\rho_{i}^{\mathbf{s}}\right)} \nabla\left((-1)^{i+1} V^{\mathbf{s}}+M_{0}^{\prime}\left(\rho_{i}^{\mathbf{s}}\right)\right)$ with $\mathbf{s}=\left(\sigma, \sigma_{1}, \Lambda_{1}\right)$, we infer from the energy estimate (2.5) and Young's inequality that $\mathcal{J}_{i}^{\mathbf{s}}$ is bounded in $L^{1}\left(\Omega_{T}\right)$ independently of all the approximation parameters. Therefore, there are vector-valued Radon measures $\mathcal{J}_{i}$ such that $\mathcal{J}_{i}^{\mathbf{s}} \rightarrow \mathcal{J}_{i}$ weakly. By Theorem 1.1 (and assuming sufficient smoothness), we may identify $\mathcal{J}_{i}:=K(\phi) \rho_{i} \nabla\left((-1)^{i+1} V+\log \rho_{i}\right)$ up to the boundary. Passing to the limit in (2.1c) (using in particular test functions not vanishing on $\partial \Omega$ ), we may establish (1.2) for $\rho_{i}, i \in\{1,2\}$, in a standard way. For the neutral species $\rho_{0}$, we argue as follows. Introducing the flux $\mathcal{J}_{0}^{\mathbf{s}}:=K\left(\phi^{\mathbf{s}}\right) \nabla \rho_{0}^{\mathbf{s}}$, we use a very weak formulation

$$
\int_{\Omega_{T}} K\left(\phi^{\mathbf{s}}\right) \nabla \rho_{0}^{\mathbf{s}} \cdot \nabla \Psi=-\int_{\Omega_{T}} K^{\prime}\left(\phi^{\mathbf{s}}\right) \nabla \phi^{\mathbf{s}} \cdot \rho_{0}^{\mathbf{s}} \nabla \Psi-\int_{\Omega_{T}} K\left(\phi^{\mathbf{s}}\right) \rho_{0}^{\mathbf{s}} \Delta \Psi
$$

for all sufficiently regular $\Psi$ with vanishing normal derivative. By Lemma 3.1, $\rho_{0}^{\mathbf{s}} \in$ $L^{5 / 3-}\left(\Omega_{T}\right)$ in all the approximation parameters. The $L^{\infty}\left(L^{6+}\right)$-regularity of $\nabla \phi^{\mathbf{s}}$ implies that we may pass to the limit in (3.12), where $K^{\prime}\left(\phi^{\mathbf{s}}\right) \nabla \phi^{\mathbf{s}} \rho_{0}^{\mathbf{s}}$ and $K\left(\phi^{\mathbf{s}}\right) \rho_{0}^{\mathbf{s}}$ weakly converge in appropriate $L^{p}$-spaces to certain weak limits. By the local strong convergences established in Lemma 3.13, Corollary 3.14, and in the proof of Theorem 1.1, an identification with $K^{\prime}(\phi) \nabla \phi \rho_{0}$ and $K(\phi) \rho_{0}$ is possible. Hence, the proof concludes as for the ion densities.

\section{Appendix A. Modeling.}

The electrokinetic equations. The main characteristic of an electrolyte is the presence of $N$ species, whose concentrations we denote by $n_{i}(\mathbf{x}, t), i=0, \ldots, N-1$, carrying a charge $\rho_{i}=e z_{i} n_{i}$, where $e$ is the charge of an electron and $z_{i}$ the valence 
of the $i$ th species. Therefore, the total charge will be given by

$$
\rho^{e}(\mathbf{x}, t)=\sum_{i=0}^{N-1} e z_{i} n_{i} .
$$

Notice that some of the species might be neutral, so that the corresponding $z_{j}$ is zero. This happens, for instance, if the fluid contains neutral species that can dissociate to produce ions of different sign or that can be produced by recombination of two ionic species.

The presence of free charges creates or modifies an imposed electric field. This is expressed by means of Poisson's equation,

$$
-\nabla \cdot\left(\varepsilon_{0} \varepsilon \nabla V\right)=\rho^{e},
$$

where $\varepsilon_{0}$ is the electric permittivity of vacuum, $\varepsilon$ the relative electric permittivity of the electrolyte medium, and $V$ the electrostatic potential so that the electric field equals $\mathbf{E}=-\nabla V$.

In the absence of dissociations or recombinations that modify the total mass of each of the species, ion and molecule concentrations satisfy the continuity equation

$$
\frac{\partial n_{i}}{\partial t}+\nabla \cdot \mathbf{j}_{i}=0
$$

where $\mathbf{j}_{i}$ is the mass flux of the $i$ th species. In response to an electric field, ions may modify their flux modeled by the Nernst-Planck law

$$
\mathbf{j}_{i}=\mu_{i} e z_{i} n_{i} \mathbf{E}-D_{i} \nabla n_{i}+n_{i} \mathbf{v}
$$

which expresses the net flux as the sum of three fluxes: the convective flux $n_{i} \mathbf{v}$ due to the velocity field $\mathbf{v}$ of the fluid, the diffusive flux $D_{i} \nabla n_{i}$, and the electrokinetic flux $\mu_{i} e z_{i} n_{i} \mathbf{E}$ due to the electric field. $\mu_{i}$ is the ion mobility: the velocity acquired by the ion when acted upon by a unit of external force. $D_{i}$ is the diffusion coefficient of the $i$ th species. Einstein's relation establishes that $\frac{D_{i}}{\mu_{i}}=R_{b} T$, where $R_{b}$ is Boltzmann's constant and $T$ the absolute temperature.

On the other hand, the motion of ions and molecules can modify the fluid flow through the forces they exert in the bulk of the fluid. Therefore, the velocity of the fluid will solve the Navier-Stokes equations

$$
\begin{aligned}
d\left(\mathbf{v}_{t}+\mathbf{v} \cdot \nabla \mathbf{v}\right) & =\nabla \cdot \mathbf{S}+\mathbf{F}_{e}, \\
\nabla \cdot \mathbf{v} & =0
\end{aligned}
$$

where $\nabla \cdot \mathbf{S}$ is a vector of components $S_{i j, i}$ and $\mathbf{F}_{e}$ is the force due to charges in the fluid. $\mathbf{S}$ is the fluid's stress tensor,

$$
S_{i j}=-p \delta_{i j}+\mu\left(v_{i, j}+v_{j, i}\right),
$$

and $d, \mu$ are the fluid's mass density and viscosity, respectively. $\mathbf{F}_{e}$ is usually expressed in terms of the so-called Maxwell stress tensor $\sigma^{M}$ as

$$
\mathbf{F}_{e}=\nabla \cdot \sigma^{M}
$$

where

$$
\sigma_{i j}^{M}=\varepsilon_{0} \varepsilon E_{i} E_{j}-\frac{1}{2} \varepsilon_{0} \varepsilon|\mathbf{E}|^{2} \delta_{i j}
$$

Copyright $@$ by SIAM. Unauthorized reproduction of this article is prohibited. 
Equivalently, using Poisson's equation,

$$
\mathbf{F}_{e}=\rho^{e} \mathbf{E}-\frac{1}{2} \varepsilon_{0}|\mathbf{E}|^{2} \nabla \varepsilon
$$

Two-phase flows and phase-field modeling. We are mostly interested in two-phase flows, that is, fluids consisting of an immiscible mixture of two fluids with different material properties $\varepsilon, \mu, d$. In this situation, continuity of the velocity field across the interface between both fluids needs to be imposed, together with balance between the jump in the total stress across the interface and surface tension forces:

$$
\left[\mathbf{S}+\sigma^{M}\right] \mathbf{n}=\gamma \kappa \mathbf{n},
$$

where $\gamma$ is the surface tension coefficient and $\kappa$ is the interface's mean curvature. Finally, appropriate boundary conditions for the velocity field have to be imposed at the boundary of the domain that contains the fluid.

In a phase-field model, which is the kind of approach that we consider in the present article, one introduces a phase-field function, such that the interface separating both fluids, instead of being a surface, is represented by the region where a function $\phi(\mathbf{x}, t)$ (the phase field) experiences drastic jumps between the two limiting values $\phi=1$ and $\phi=-1$ representing the two different fluids, respectively. In this fashion, material properties that change from one fluid to the other can be represented as functions of $\phi: \varepsilon(\phi), \mu(\phi), d(\phi)$. The Navier-Stokes equations can be defined in both fluids simultaneously, and the boundary condition (A.2) does not need to be imposed. Instead, a bulk force associated to surface tension needs to be added to Navier-Stokes equations. This force is of the form

$$
\mathbf{F}_{s t}=\nu[\phi] \nabla \phi,
$$

where $\frac{\nabla \phi}{|\nabla \phi|}$ is obviously orthogonal to the level lines of $\phi$ and therefore a representation of $\mathbf{n}$, while $\nu[\phi]$ is a certain second order differential operator providing an approximation of the surface's mean curvature. Compared to other modeling approaches for multiphase flow, phase-field models are distinguished by their capability to model topology changes easily.

Moreover, consistency with the second law of thermodynamics may be established as well. In this spirit, we will derive an expression for approximated curvatures by means of energetic considerations, too. This strategy has already been pursued in [11] for a model assuming ohmic charge conduction. The main idea consists of replacing the interfacial surface energy

$$
F_{s t}=\gamma A,
$$

where $A$ is the interface's area, by

$$
F_{s t}=\gamma_{0} \int \delta \frac{|\nabla \phi|^{s}}{s}+\frac{1}{\delta} W(\phi)
$$

where $s \geq 2, \delta \ll 1$, and $W(\phi)=\left(1-\phi^{2}\right)^{2}$. In the literature, usually $s=2$ is considered. The idea of taking $s>2$ is from Abels (see [2]), and for $\delta \rightarrow 0$, the energy in (A.4) has the same limit behavior as the classical one with $s=2$. While the first variation of the energy (A.3) with respect to infinitesimal variations $\Delta \mathrm{x}$ of the interface profile is

$$
\Delta F_{s t}=\gamma \int_{\text {interface }} \kappa \mathbf{n} \cdot \Delta \mathbf{x}
$$


the first variation of the energy (A.4) with respect to an increment $\widetilde{\phi}$ is

$$
\Delta F_{s t}=\gamma_{0} \int_{\text {interface }}\left(-\delta \nabla \cdot\left(|\nabla \phi|^{s-2} \nabla \phi\right)+\frac{1}{\delta} W^{\prime}(\phi)\right) \widetilde{\phi} .
$$

This leads to the definition

$$
\nu[\phi]=\gamma_{0}\left(-\delta \nabla \cdot\left(|\nabla \phi|^{s-2} \nabla \phi\right)+\frac{1}{\delta} W^{\prime}(\phi)\right) .
$$

Finally, an equation must be given for the evolution of the interface. This is simply the condition that the interface is formed by material particles; i.e., they move following the flow's velocity field. In terms of the phase field $\phi$, this amounts to requiring that a certain level line representing the interface be convected by $\mathbf{v}$. Instead of taking a purely convective equation for $\phi$, it is customary to write a CahnHilliard-type equation of the form

$$
\phi_{t}+\mathbf{v} \cdot \nabla \phi=\nabla \cdot(M(\phi) \nabla \mu)
$$

which enjoys much better regularity properties while ensuring conservation of mass of the order parameter. $M(\phi)$ is a smooth function, called the mobility function, and $\mu$ is the chemical potential. In the simplest phase-field models, the chemical potential $\mu$ is simply $\nu[\phi]$ given by (A.5). For the present model, where Cahn-Hilliard and Navier-Stokes are coupled with electrokinetic equations, it was shown in [11] that $\mu$ must be taken as

$$
\mu=\gamma_{0}\left(-\delta \nabla \cdot\left(|\nabla \phi|^{s-2} \nabla \phi\right)+\frac{1}{\delta} W^{\prime}(\phi)\right)-\frac{1}{2} \varepsilon_{0} \varepsilon^{\prime}(\phi)|\mathbf{E}|^{2} .
$$

The phase-field model for binary $z$-z electrolytes. In this article, attention is focused on liquids with charge from a single 1-1 electrolyte. This involves three species: one neutral molecule plus two ions with valence 1 . Think, for instance, of salt $(\mathrm{NaCl})$ molecules that can dissociate into two ions with charge +1 and -1 , respectively $\left(\mathrm{Na}^{+}\right.$ and $\mathrm{Cl}^{-}$). Hence, there are two homogeneous reactions, a forward reaction producing positive and negative ions from dissociation of the neutral species, and a recombination of the two ions to produce a neutral molecule. These reactions give rise to terms on the r.h.s. of (A.1), depending on the reaction rates of dissociation and recombination processes. If we call the density of the neutral species $\rho_{0}$ and the charge densities of the ion species $\rho_{1}, \rho_{2}$, then the dissociation process is produced at a rate $k_{0} \rho_{0}^{+}$, and the recombination process at a rate $k_{1} \rho_{1}^{+} \rho_{2}^{+}$, respectively. The parameters $k_{0}$ and $k_{1}$ depend on the medium in which the reactions take place, i.e., on $\phi$, and may also depend on the species concentrations. Here, we will consider $k_{1}=K_{1}(\phi)$ and $k_{0}=K_{0}(\phi) \rho_{0}^{\alpha-1}$ with $0<\alpha<1$. This choice of $\alpha$ is mainly due to the technical limitations of the analytical approach presented in this work, but has a clear physical meaning: we limit the capacity of neutral molecules to dissociate at very large values of $\rho_{0}$ so that, instead of growing exponentially in absence of recombination or other processes, the density may grow only as a power law. More generally, we may admit nonnegative functions $k_{0}(\phi, \rho):=K_{0}(\phi) \cdot f(\rho) \cdot \rho$ such that $f(\rho) \cdot \rho^{1-\alpha} \leq C$ for all $\rho \in \mathbb{R}_{0}^{+}$. This includes in particular linear dissociation behavior for sufficiently small $\rho$.

An additional assumption is that both fluids have matched mass densities that we can consider, without loss of generality, to be unit. 
To summarize, the equations we consider are as follows: the Navier-Stokes equations

$$
\begin{aligned}
\mathbf{v}_{t}+(\mathbf{v} \cdot \nabla) \mathbf{v}-\nabla \cdot(\eta(\phi) \mathbf{T}(\mathbf{v}))+\nabla p=\left(\rho_{1}-\rho_{2}\right) \mathbf{E}+\mu \nabla \phi \text { in } \Omega_{T}, \\
\nabla \cdot \mathbf{v}=0 \text { in } \Omega_{T},
\end{aligned}
$$

where $\mathbf{T}(\mathbf{v})$ is the tensor of components $\frac{1}{2}\left(v_{i, j}+v_{j, i}\right), \eta(\phi)$ is the kinematic viscosity, and $\mu$ is the chemical potential defined in (A.7). Notice that the force $\left(\rho_{1}-\rho_{2}\right) \mathbf{E}+\mu \nabla \phi$ is then the sum of the electrostatic force,

$$
\mathbf{F}_{e}=\left(\rho_{1}-\rho_{2}\right) \mathbf{E}-\frac{1}{2} \varepsilon_{0} \varepsilon^{\prime}(\phi)|\mathbf{E}|^{2} \nabla \phi,
$$

and the force due to surface tension,

$$
\mathbf{F}_{s t}=\gamma_{0}\left(-\delta \nabla \cdot\left(|\nabla \phi|^{s-2} \nabla \phi\right)+\frac{1}{\delta} W^{\prime}(\phi)\right) \nabla \phi .
$$

Since $\mathbf{E}$ is a curl-free field (magnetic effects are usually neglected in electrokinetics), we can write $\mathbf{E}=-\nabla V$.

The density of neutral species will satisfy the equation

$$
\rho_{0, t}+\mathbf{v} \cdot \nabla \rho_{0}=\nabla \cdot\left(K(\phi) \frac{R_{b} T}{e} \nabla \rho_{0}\right)-K_{0}(\phi)\left(\rho_{0}^{+}\right)^{\alpha}+K_{1}(\phi) \rho_{1}^{+} \rho_{2}^{+} \text {in } \Omega_{T},
$$

and the densities of positive and negative ions the equations

$$
\begin{aligned}
\rho_{i, t} & +\mathbf{v} \cdot \nabla \rho_{i}=\nabla \cdot\left(K(\phi) \rho_{i}^{+} \nabla\left((-1)^{i+1} V+\frac{R_{b} T}{e} \log \rho_{i}\right)\right) \\
& +K_{0}(\phi)\left(\rho_{0}^{+}\right)^{\alpha}-K_{1}(\phi) \rho_{1}^{+} \rho_{2}^{+} \quad \text { in } \Omega_{T}
\end{aligned}
$$

for $i \in\{1,2\}$, respectively. The coefficient $K(\phi)$ is proportional to the diffusion coefficient, hence also to the mobility, and depends in the medium on $\phi$. Suitable rescaling of the densities allows us to eliminate the factor $\frac{R_{b} T}{e}$ from the equations and, for the sake of simplicity, we will take it always equal to 1 as well as $\delta, \varepsilon_{0}$, and $\gamma_{0}$.

The electric field $\mathbf{E}$ satisfies Poisson's equation,

$$
\nabla \cdot\left(\varepsilon_{0} \varepsilon(\phi) \mathbf{E}\right)=\rho_{1}-\rho_{2} \text { in } \Omega^{*} \text { for all } t \in(0, T),
$$

and the phase field $\phi$ satisfies the Cahn-Hilliard equation (A.6) in $\Omega_{T}$. Therefore, the model consists of (A.6)-(A.12) with unknowns $\phi, \mu, \mathbf{v}, p, \rho_{0}, \rho_{1}, \rho_{2}$, and $V$.

Appendix B. Existence results for a basic auxiliary problem. In this section, existence for a basic auxiliary problem is established.

Theorem B.1. Let assumptions $\left(A_{1}\right)-\left(A_{8}\right)$ hold. For given initial data $\mathbf{v}_{0} \in \mathcal{V}$, $\rho_{i, 0} \in L^{2}(\Omega)$ with $\rho_{i, 0} \geq 0$ a.e. in $\Omega, i \in\{0,1,2\}, \phi_{0} \in W^{1, s}(\Omega)$, there exists a septuple $\left(\mathbf{v}, \rho_{0}, \rho_{1}, \rho_{2}, \phi, \mu, V\right)$ such that for any $\beta<\frac{5}{6}$ (all bounds depend on $\sigma, \sigma_{1}, \sigma_{2}, \sigma_{3}, L, \Lambda_{1}$, and $\Lambda_{2}$ )

$$
\begin{aligned}
\mathbf{v} & \in L^{\infty}(I ; \mathcal{V}) \cap H^{1}(I ; \mathcal{V}), \\
\rho_{i} & \in L^{\infty}\left(I ; L^{2}(\Omega)\right) \cap L^{2}\left(I ; H^{1}(\Omega)\right) \cap H^{1}\left(I ;\left(H^{1}(\Omega)\right)^{\prime}\right) \text { for } i \in\{0,1,2\}, \\
\phi & \in L^{\infty}\left(I ; W^{1, s}(\Omega)\right) \cap C^{0}\left(\bar{I} ; C^{0, \beta}(\bar{\Omega})\right) \cap H^{1}\left(I ; L^{2}(\partial \Omega)\right) \cap H^{1}\left(I ;\left(H^{1}(\Omega)\right)^{\prime}\right), \\
V-\bar{V} & \in L^{\infty}\left(I ; H_{0}^{1}\left(\Omega^{*}\right)\right), \\
\mu & \in L^{2}\left(I ; H^{1}(\Omega)\right),
\end{aligned}
$$

Copyright (c) by SIAM. Unauthorized reproduction of this article is prohibited. 
which solves the following weak system of partial differential equations:

$$
\begin{aligned}
\int_{\Omega_{T}}\left(\left(\mathbf{v}_{t}+(\mathbf{v} \cdot \nabla) \mathbf{v}\right) \cdot \mathbf{w}+\sigma_{1} \nabla \mathbf{v}_{t} \cdot \nabla \mathbf{w}+\eta(\phi) \mathbf{T}(\mathbf{v}): \mathbf{T}(\mathbf{w})\right) & \\
+ & \int_{\Omega_{T}}\left(\left(\frac{\rho_{1}^{+}}{1+\sigma \rho_{1}^{+}}-\frac{\rho_{2}^{+}}{1+\sigma \rho_{2}^{+}}\right) \nabla V-\mu \nabla \phi\right) \cdot \mathbf{w}=0 \\
\int_{\Omega_{T}} \rho_{0, t} \psi_{1} & +\int_{\Omega_{T}}\left(K(\phi) \nabla \rho_{0}-\rho_{0} \mathbf{v}\right) \cdot \nabla \psi_{1} \\
& +\int_{\Omega_{T}}\left(K_{0}(\phi)\left(\rho_{0}^{+}\right)^{\alpha}-K_{1}(\phi) \bar{M}_{\Lambda_{1}}\left(\rho_{1}^{+}\right) \bar{M}_{\Lambda_{1}}\left(\rho_{2}^{+}\right)\right) \psi_{1}=0
\end{aligned}
$$

$$
\begin{aligned}
\int_{\Omega_{T}}\left(\rho_{i, t}\right. & \left.+\sigma_{3}\left((-1)^{i+1} V+M_{L}^{\prime}\left(\rho_{i}\right)\right)\right) \psi_{2}+\int_{\Omega_{T}} \sigma_{2} \nabla \rho_{i} \cdot \nabla \psi_{2} \\
& +\int_{\Omega_{T}}\left(K(\phi) \frac{1}{M_{L}^{\prime \prime}\left(\rho_{i}\right)} \nabla\left((-1)^{i+1} V+M_{L}^{\prime}\left(\rho_{i}\right)\right)-\frac{\rho_{i}^{+}}{1+\sigma \rho_{i}^{+}} \mathbf{v}\right) \cdot \nabla \psi_{2} \\
& +\int_{\Omega_{T}}\left(-K_{0}(\phi)\left(\rho_{0}^{+}\right)^{\alpha}+K_{1}(\phi) \bar{M}_{\Lambda_{2}}\left(\rho_{1}^{+}\right) \bar{M}_{\Lambda_{2}}\left(\rho_{2}^{+}\right)\right) \psi_{2}=0 \text { for } i \in\{1,2\},
\end{aligned}
$$

$$
\begin{gathered}
\int_{\Omega_{T}}\left(\phi_{t}+\mathbf{v} \cdot \nabla \phi\right) \psi_{3}+\int_{\Omega_{T}} M(\phi) \nabla \mu \cdot \nabla \psi_{3}=0 \\
\int_{\Omega_{T}} \mu \psi_{4}=\int_{\Omega_{T}}\left(|\nabla \phi|^{s-2} \nabla \phi \cdot \nabla \psi_{4}+W^{\prime}(\phi) \psi_{4}-\frac{1}{2} \varepsilon^{\prime}(\phi)|\nabla V|^{2} \psi_{4}\right) \\
+\int_{0}^{T} \int_{\partial \Omega}\left(\alpha \phi_{t}+\gamma_{f s}^{\prime}(\phi)\right) \psi_{4}, \\
\int_{\Omega_{T}^{*}} \bar{\varepsilon}[\phi] \nabla V \cdot \nabla \chi=\int_{\Omega_{T}}\left(\rho_{1}-\rho_{2}\right) \chi
\end{gathered}
$$

for every $\mathbf{w} \in L^{2}(I ; \mathcal{V}), \psi_{1} \in L^{2}\left(I ; H^{1}(\Omega)\right), \psi_{2} \in L^{2}\left(I ; H^{1}(\Omega)\right), \psi_{3} \in L^{2}\left(I ; H^{1}(\Omega)\right)$, $\psi_{4} \in L^{s}\left(I ; W^{1, s}(\Omega)\right) \cap H^{1}\left(I ; L^{2}(\partial \Omega)\right)$, and $\chi \in L^{2}(I ; \mathcal{U})$. Here, $\sigma, \sigma_{1}, \sigma_{2}, \sigma_{3}, L<1<$ $\Lambda_{1}, \Lambda_{2}$ are approximation parameters, $\bar{M}_{\Lambda}(x)=\min (x, \Lambda)$, and the regularized ion free energy contribution is

$$
M_{L}(\rho)= \begin{cases}\rho \log \rho-\rho+\frac{\sigma}{2} \rho^{2}, & \rho>L, \\ \frac{1}{2} \frac{1+\sigma L}{L} \rho^{2}+(\log L-1) \rho-\frac{L}{2}, & \rho \leq L .\end{cases}
$$

Proof. Discrete existence result. The first step is to prove existence for an appropriate discrete system using the Faedo-Galerkin method. For this, define finitedimensional subspaces

$$
\begin{aligned}
\mathcal{V}_{n} & :=\operatorname{span}\left\{\mathbf{w}_{1}, \mathbf{w}_{2}, \mathbf{w}_{3}, \ldots, \mathbf{w}_{n}\right\} \text { of } \mathcal{V}, \\
\mathcal{W}_{n} & :=\operatorname{span}\left\{\psi_{1}, \psi_{2}, \psi_{3}, \ldots, \psi_{n}\right\} \text { of } \mathcal{W} \\
\mathcal{W}_{n}^{*} & :=\operatorname{span}\left\{\psi_{1}^{*}, \psi_{2}^{*}, \psi_{3}^{*}, \ldots, \psi_{n}^{*}\right\} \text { of } W^{1, s}(\Omega), \\
\mathcal{U}_{n} & :=\operatorname{span}\left\{\chi_{1}, \chi_{2}, \chi_{3}, \ldots, \chi_{n}\right\} \text { of } \mathcal{U}
\end{aligned}
$$

Copyright $@$ by SIAM. Unauthorized reproduction of this article is prohibited. 
Here, $\mathbf{w}_{i}, i \in \mathbb{N}$, form a complete system of $L^{2}$-orthonormal eigenfunctions of the Stokes operator with homogeneous Dirichlet boundary conditions. Similarly, $\psi_{i}, \chi_{i}$, $i \in \mathbb{N}$, are a basis of $L^{2}$-orthonormal eigenfunctions of the Laplace operator with homogeneous Neumann or Dirichlet boundary conditions, respectively. It is well known that the spaces $\bigcup_{n \in \mathbb{N}} \mathcal{V}_{n}, \bigcup_{n \in \mathbb{N}} \mathcal{W}_{n}, \bigcup_{n \in \mathbb{N}} \mathcal{U}_{n}$ are dense in $\mathcal{V}, \mathcal{W}, \mathcal{U}$, respectively. Note that due to the dense embedding of $H^{3}(\Omega)$ into $W^{1, s}(\Omega)$, the Neumann basis functions of $H^{3}(\Omega)$ build a complete system of $W^{1, s}(\Omega)$. Under condition $\left(A_{1}\right)$ for the domains $\Omega$ and $\Omega^{*}$, the elements of $\mathcal{V}_{n}, \mathcal{W}_{n}, \mathcal{W}_{n}^{*}, \mathcal{U}_{n}$ are bounded. The solutions to be computed are approximated by elements of these spaces,

$$
\begin{aligned}
\mathbf{v}(t, x) \sim \mathbf{v}^{(n)}(t, x) & :=\sum_{j=1}^{n} \hat{v}_{j}^{(n)}(t) \mathbf{w}_{j}(x), \\
\rho_{i}(t, x) \sim \rho_{i}^{(n)}(t, x) & :=\sum_{j=1}^{n} \hat{\rho}_{i, j}^{(n)}(t) \psi_{j}(x) \text { for } i \in\{0,1,2\}, \\
\phi(t, x) \sim \phi^{(n)}(t, x) & :=\sum_{j=1}^{n} \hat{\phi}_{j}^{(n)}(t) \psi_{j}^{*}(x), \\
\mu(t, x) \sim \mu^{(n)}(t, x) & :=\sum_{j=1}^{n} \hat{\mu}_{j}^{(n)}(t) \psi_{j}^{*}(x), \\
V(t, x) \sim V^{(n)}(t, x) & :=\bar{V}(t, x)+\sum_{j=1}^{n} \hat{V}_{j}^{(n)}(t) \chi_{j}(x) .
\end{aligned}
$$

Generally, we denote Galerkin functions and their coefficients by the same letter and emphasize coefficients by ^symbols.

Using the Picard-Lindelöf theorem, local-in-time existence for the following Galerkin system can be proven in a way which is very similar to [11, Chap. 3.3] such that we skip the proof here.

Find $\mathbf{v}^{(n)} \in C^{1}\left(\bar{I} ; \mathcal{V}_{n}\right), \rho_{i}^{(n)} \in C^{1}\left(\bar{I} ; \mathcal{W}_{n}\right)$ for $i \in\{0,1,2\}, \phi^{(n)} \in C^{1}\left(\bar{I} ; \mathcal{W}_{n}^{*}\right)$, $\mu^{(n)} \in C^{0}\left(\bar{I} ; \mathcal{W}_{n}^{*}\right), V^{(n)} \in C^{0}\left(\bar{I} ; \mathcal{U}_{n}\right)$ such that for every $t \in \bar{I}, \mathbf{w} \in \mathcal{V}_{n}, \psi_{1}, \psi_{2}, \in \mathcal{W}_{n}$, $\psi_{3}, \psi_{4} \in \mathcal{W}_{n}^{*}, \chi \in \mathcal{U}_{n}$

$$
\begin{aligned}
\int_{\Omega} & {\left[\mathbf{v}_{t}^{(n)} \cdot \mathbf{w}+\left(\mathbf{v}^{(n)} \cdot \nabla\right) \mathbf{v}^{(n)} \cdot \mathbf{w}+\eta\left(\phi^{(n)}\right) \mathbf{T}\left(\mathbf{v}^{(n)}\right): \mathbf{T}(\mathbf{w})\right.} \\
& -\mu^{(n)} \nabla \phi^{(n)} \cdot \mathbf{w}+\sigma_{1} \nabla \mathbf{v}_{t}^{(n)} \cdot \nabla \mathbf{w}+\left\{\frac{\left(\rho_{1}^{(n)}\right)^{+}}{1+\sigma\left(\rho_{1}^{(n)}\right)^{+}} \nabla\left[P_{n}\left(V^{(n)}+M_{L}^{\prime}\left(\rho_{1}^{(n)}\right)\right)\right]\right. \\
& \left.\left.+\frac{\left(\rho_{2}^{(n)}\right)^{+}}{1+\sigma\left(\rho_{2}^{(n)}\right)^{+}} \nabla\left[P_{n}\left(-V^{(n)}+M_{L}^{\prime}\left(\rho_{2}^{(n)}\right)\right)\right]\right\} \cdot \mathbf{w}\right]=0,
\end{aligned}
$$

$$
\begin{aligned}
& \int_{\Omega}\left[\rho_{0, t}^{(n)} \psi_{1}+\left(-\rho_{0}^{(n)} \mathbf{v}^{(n)}+K\left(\phi^{(n)}\right) \nabla \rho_{0}^{(n)}\right) \cdot \nabla \psi_{1}+K_{0}\left(\phi^{(n)}\right)\left(\left(\rho_{0}^{(n)}\right)^{+}\right)^{\alpha} \psi_{1}\right] \\
& \quad=\int_{\Omega} K_{1}\left(\phi^{(n)}\right) \bar{M}_{\Lambda_{1}}\left(\left(\rho_{1}^{(n)}\right)^{+}\right) \bar{M}_{\Lambda_{1}}\left(\left(\rho_{2}^{(n)}\right)^{+}\right) \psi_{1},
\end{aligned}
$$

Copyright $@$ by SIAM. Unauthorized reproduction of this article is prohibited. 


$$
\begin{gathered}
\int_{\Omega}\left[\rho_{i, t}^{(n)} \psi_{2}+\sigma_{2} \nabla \rho_{i}^{(n)} \cdot \nabla \psi_{2}+\sigma_{3} P_{n}\left((-1)^{i+1} V^{(n)}+M_{L}^{\prime}\left(\rho_{i}^{(n)}\right)\right) \psi_{2}\right. \\
+K\left(\phi^{(n)}\right) \frac{1}{M_{L}^{\prime \prime}\left(\rho_{i}^{(n)}\right)} \nabla\left[P_{n}\left((-1)^{i+1} V^{(n)}+M_{L}^{\prime}\left(\rho_{i}^{(n)}\right)\right)\right] \cdot \nabla \psi_{2} \\
\left.-\frac{\left(\rho_{i}^{(n)}\right)^{+}}{1+\sigma\left(\rho_{i}^{(n)}\right)^{+}} \mathbf{v}^{(n)} \cdot \nabla \psi_{2}\right]=\int_{\Omega}\left[K_{0}\left(\phi^{(n)}\right)\left(\left(\rho_{0}^{(n)}\right)^{+}\right)^{\alpha}\right. \\
\left.-K_{1}\left(\phi^{(n)}\right) \bar{M}_{\Lambda_{2}}\left(\left(\rho_{1}^{(n)}\right)^{+}\right) \bar{M}_{\Lambda_{2}}\left(\left(\rho_{2}^{(n)}\right)^{+}\right)\right] \psi_{2} \quad \text { for } i \in\{1,2\} \\
\int_{\Omega}\left[\phi_{t}^{(n)} \psi_{3}+\mathbf{v}^{(n)} \cdot \nabla \phi^{(n)} \psi_{3}+M\left(\phi^{(n)}\right) \nabla \mu^{(n)} \cdot \nabla \psi_{3}\right]=0, \\
\int_{\Omega} \mu^{(n)} \psi_{4}=\int_{\Omega}\left[\left|\nabla \phi^{(n)}\right|^{s-2} \nabla \phi^{(n)} \cdot \nabla \psi_{4}+W^{\prime}\left(\phi^{(n)}\right) \psi_{4}\right. \\
\left.-\frac{1}{2} \varepsilon^{\prime}\left(\phi^{(n)}\right)\left|\nabla V^{(n)}\right|^{2} \psi_{4}\right]+\int_{\partial \Omega}\left[\alpha \phi_{t}^{(n)}+\gamma_{f s}^{\prime}\left(\phi^{(n)}\right)\right] \psi_{4}, \\
\int_{\Omega^{*}} \bar{\varepsilon}\left[\phi^{(n)}\right] \nabla V^{(n)} \cdot \nabla \chi=\int_{\Omega}\left(\rho_{1}^{(n)}-\rho_{2}^{(n)}\right) \chi
\end{gathered}
$$

Here, $P_{n}: L^{2}(\Omega) \rightarrow \mathcal{W}_{n}$ denotes the orthogonal $L^{2}$-projection.

A priori estimates for the discrete solutions. Testing in (B.2b) by $\psi_{1}=\rho_{0}^{(n)}$ and integrating with respect to time from 0 up to some $t_{0}$, we get

$$
\begin{gathered}
{\left[\int_{\Omega} \frac{1}{2}\left(\rho_{0}^{(n)}\right)^{2}\right]_{0}^{t_{0}}+\int_{\Omega_{t_{0}}} K\left(\phi^{(n)}\right)\left|\nabla \rho_{0}^{(n)}\right|^{2}+\int_{\Omega_{t_{0}}} K_{0}\left(\phi^{(n)}\right)\left(\left(\rho_{0}^{(n)}\right)^{+}\right)^{\alpha+1}} \\
=\int_{\Omega_{t_{0}}} K_{1}\left(\phi^{(n)}\right) \bar{M}_{\Lambda_{1}}\left(\left(\rho_{1}^{(n)}\right)^{+}\right) \bar{M}_{\Lambda_{1}}\left(\left(\rho_{2}^{(n)}\right)^{+}\right) \rho_{0}^{(n)} .
\end{gathered}
$$

Now, Gronwall's inequality yields

$$
\rho_{0}^{(n)} \in L^{\infty}\left(0, t_{0} ; L^{2}(\Omega)\right) \cap L^{2}\left(0, t_{0} ; H^{1}(\Omega)\right) \text { uniformly in } n \in \mathbb{N}
$$

but, of course, with a bound depending on $\Lambda_{1}$ and on time. Taking the time derivative of (B.2f) for time-independent $\chi$ leads to

$$
\int_{\Omega^{*}} \frac{d}{d t}\left(\bar{\varepsilon}\left[\phi^{(n)}\right] \nabla V^{(n)}\right) \cdot \nabla \chi=\int_{\Omega^{*}}\left(\rho_{1, t}^{(n)}-\rho_{2, t}^{(n)}\right) \chi .
$$

For a test function $\chi=V^{(n)}-\bar{V}$, we obtain

$$
\begin{aligned}
\int_{\Omega^{*}} & {\left[\frac{d}{d t}\left(\frac{1}{2} \bar{\varepsilon}\left[\phi^{(n)}\right]\left|\nabla V^{(n)}\right|^{2}\right)+\frac{1}{2} \varepsilon^{\prime}\left(\phi^{(n)}\right) \phi_{t}^{(n)}\left|\nabla V^{(n)}\right|^{2}-\left(\rho_{1, t}^{(n)}-\rho_{2, t}^{(n)}\right) V^{(n)}\right] } \\
= & \int_{\Omega^{*}}\left[\frac{d}{d t}\left(\bar{\varepsilon}\left[\phi^{(n)}\right] \nabla V^{(n)}\right) \cdot \nabla \bar{V}-\left(\rho_{1, t}^{(n)}-\rho_{2, t}^{(n)}\right) \bar{V}\right] .
\end{aligned}
$$

Taking $\mathbf{w}=\mathbf{v}^{(n)}$ in (B.2a), $\psi_{2}=P_{n}\left((-1)^{i+1} V^{(n)}+M_{L}^{\prime}\left(\rho_{i}^{(n)}\right)\right)$ in (B.2c) for $i \in\{1,2\}$, respectively, $\psi_{3}=\mu^{(n)}$ in (B.2d), $\psi_{4}=\phi_{t}^{(n)}$ in (B.2e), and adding the results to (B.5)

Copyright $@$ by SIAM. Unauthorized reproduction of this article is prohibited. 
yields

$$
\begin{aligned}
& \frac{d}{d t}\left[\int _ { \Omega } \left\{\frac{1}{2}\left|\mathbf{v}^{(n)}\right|^{2}+\frac{\sigma_{1}}{2}\left|\nabla \mathbf{v}^{(n)}\right|^{2}+M_{L}\left(\rho_{1}^{(n)}\right)+M_{L}\left(\rho_{2}^{(n)}\right)+\frac{1}{s}\left|\nabla \phi^{(n)}\right|^{s}\right.\right. \\
& \left.\left.+W\left(\phi^{(n)}\right)\right\}+\int_{\partial \Omega} \gamma_{f s}\left(\phi^{(n)}\right)+\int_{\Omega^{*}} \frac{1}{2} \bar{\varepsilon}\left[\phi^{(n)}\right]\left|\nabla V^{(n)}\right|^{2}\right] \\
& +\int_{\Omega}\left[\eta\left(\phi^{(n)}\right)\left|\mathbf{T}\left(\mathbf{v}^{(n)}\right)\right|^{2}+\sigma_{3}\left|P_{n}\left(V^{(n)}+M_{L}^{\prime}\left(\rho_{1}^{(n)}\right)\right)\right|^{2}\right. \\
& +\sigma_{3}\left|P_{n}\left(-V^{(n)}+M_{L}^{\prime}\left(\rho_{2}^{(n)}\right)\right)\right|^{2} \\
& +K\left(\phi^{(n)}\right) \frac{1}{M_{L}^{\prime \prime}\left(\rho_{1}^{(n)}\right)}\left|\nabla\left[P_{n}\left(V^{(n)}+M_{L}^{\prime}\left(\rho_{1}^{(n)}\right)\right)\right]\right|^{2} \\
& +K\left(\phi^{(n)}\right) \frac{1}{M_{L}^{\prime \prime}\left(\rho_{2}^{(n)}\right)}\left|\nabla\left[P_{n}\left(-V^{(n)}+M_{L}^{\prime}\left(\rho_{2}^{(n)}\right)\right)\right]\right|^{2} \\
& \left.+M\left(\phi^{(n)}\right)\left|\nabla \mu^{(n)}\right|^{2}\right]+\int_{\partial \Omega} \alpha\left|\phi_{t}^{(n)}\right|^{2} \\
& =\int_{\Omega^{*}}\left[\frac{d}{d t}\left(\bar{\varepsilon}\left[\phi^{(n)}\right] \nabla V^{(n)}\right) \cdot \nabla \bar{V}-\left(\rho_{1, t}^{(n)}-\rho_{2, t}^{(n)}\right) \bar{V}\right] \\
& -\sigma_{2} \int_{\Omega} \nabla \rho_{1}^{(n)} \cdot \nabla\left[P_{n}\left(V^{(n)}+M_{L}^{\prime}\left(\rho_{1}^{(n)}\right)\right)\right] \\
& -\sigma_{2} \int_{\Omega} \nabla \rho_{2}^{(n)} \cdot \nabla\left[P_{n}\left(-V^{(n)}+M_{L}^{\prime}\left(\rho_{2}^{(n)}\right)\right)\right] \\
& -\int_{\Omega} K_{1}\left(\phi^{(n)}\right) \bar{M}_{\Lambda_{2}}\left(\left(\rho_{1}^{(n)}\right)^{+}\right) \bar{M}_{\Lambda_{2}}\left(\left(\rho_{2}^{(n)}\right)^{+}\right) P_{n}\left(V^{(n)}+M_{L}^{\prime}\left(\rho_{1}^{(n)}\right)\right) \\
& -\int_{\Omega} K_{1}\left(\phi^{(n)}\right) \bar{M}_{\Lambda_{2}}\left(\left(\rho_{1}^{(n)}\right)^{+}\right) \bar{M}_{\Lambda_{2}}\left(\left(\rho_{2}^{(n)}\right)^{+}\right) P_{n}\left(-V^{(n)}+M_{L}^{\prime}\left(\rho_{2}^{(n)}\right)\right) \\
& +\int_{\Omega} K_{0}\left(\phi^{(n)}\right)\left(\left(\rho_{0}^{(n)}\right)^{+}\right)^{\alpha} P_{n}\left(V^{(n)}+M_{L}^{\prime}\left(\rho_{1}^{(n)}\right)\right) \\
& +\int_{\Omega} K_{0}\left(\phi^{(n)}\right)\left(\left(\rho_{0}^{(n)}\right)^{+}\right)^{\alpha} P_{n}\left(-V^{(n)}+M_{L}^{\prime}\left(\rho_{2}^{(n)}\right)\right) .
\end{aligned}
$$

Next, we integrate this equation with respect to time over $\left(0, t_{0}\right)$ with some $t_{0}$ and estimate the r.h.s. independently of $n \in \mathbb{N}$, but dependent on all the other approximation parameters. For this, we integrate the first term on the r.h.s. by parts,

$$
\begin{aligned}
\int_{\Omega_{t_{0}}^{*}} & {\left[\frac{d}{d t}\left(\bar{\varepsilon}\left[\phi^{(n)}\right] \nabla V^{(n)}\right) \cdot \nabla \bar{V}-\left(\rho_{1, t}^{(n)}-\rho_{2, t}^{(n)}\right) \bar{V}\right] } \\
= & {\left[\int_{\Omega^{*}}\left(\bar{\varepsilon}\left[\phi^{(n)}\right] \nabla V^{(n)} \cdot \nabla \bar{V}-\left(\rho_{1}^{(n)}-\rho_{2}^{(n)}\right) \bar{V}\right)\right]_{0}^{t_{0}} } \\
& -\int_{\Omega_{t_{0}}^{*}}\left[\left(\bar{\varepsilon}\left[\phi^{(n)}\right] \nabla V^{(n)}\right) \cdot \nabla \bar{V}_{t}-\left(\rho_{1}^{(n)}-\rho_{2}^{(n)}\right) \bar{V}_{t}\right] .
\end{aligned}
$$

Note that our particular choice of the Galerkin space $\mathcal{W}_{n}$ - together with an orthogonality argument - allows us to identify the second and third terms on the r.h.s. with

$$
(-1)^{i+1} \sigma_{2} \int_{\Omega} \nabla \rho_{i}^{(n)} \cdot \nabla V^{(n)}-\sigma_{2} \int_{\Omega} M_{L}^{\prime \prime}\left(\rho_{i}\right)\left|\nabla \rho_{i}^{(n)}\right|^{2}, \quad i=1,2 .
$$

Copyright $@$ by SIAM. Unauthorized reproduction of this article is prohibited. 
Using the inequalities of Gronwall, Hölder, and Young several times, as well as Poincaré's and Korn's inequalities for $\mathbf{v}^{(n)}$ and some standard absorption techniques in combination with the assumptions on initial data and on $\bar{V}$, we get the following bounds uniformly in $n \in \mathbb{N}$, but depending on time and $\sigma, \sigma_{3}, L, \Lambda_{1}, \Lambda_{2}$ :

$$
\begin{aligned}
& \mathbf{v}^{(n)} \in L^{\infty}\left(I ; L^{2}(\Omega)\right) \cap L^{2}\left(I ; H^{1}(\Omega)\right), \quad \sigma_{1}^{1 / 2} \mathbf{v}^{(n)} \in L^{\infty}\left(I ; H^{1}(\Omega)\right), \\
& \rho_{i}^{(n)} \in L^{\infty}\left(I ; L^{2}(\Omega)\right), \quad \sigma_{2}^{1 / 2} \rho_{i}^{(n)} \in L^{2}\left(I ; H^{1}(\Omega)\right) \text { for } i \in\{1,2\}, \\
& \phi^{(n)} \in L^{\infty}\left(I ; W^{1, s}(\Omega)\right), \quad V^{(n)} \in L^{\infty}\left(I ; H^{1}\left(\Omega^{*}\right)\right), \quad \mu^{(n)} \in L^{2}\left(I ; H^{1}(\Omega)\right) .
\end{aligned}
$$

The $L^{\infty}$-bounds for $\phi^{(n)}, \rho_{0}^{(n)}, \rho_{1}^{(n)}, \rho_{2}^{(n)}$, and $\mathbf{v}^{(n)}$ imply that the solutions to (B.2) exist globally in time.

Compactness in time and convergence results. Let us next prove regularity with respect to time. For the velocity field, we test (B.2a) by $\mathbf{w}=\mathbf{v}_{t}^{(n)}$. Note that

$$
\begin{aligned}
& \int_{\Omega_{T}} \eta\left(\phi^{(n)}\right) \mathbf{T}\left(\mathbf{v}^{(n)}\right): \mathbf{T}\left(\mathbf{v}_{t}^{(n)}\right)=\int_{\Omega_{T}} \eta\left(\phi^{(n)}\right) \frac{\partial}{\partial t} \frac{1}{2}\left|\mathbf{T}\left(\mathbf{v}^{(n)}\right)\right|^{2} \\
= & \int_{\Omega_{T}}\left(\eta\left(\phi^{(n)}\right)-\eta_{-}\right) \frac{\partial}{\partial t} \frac{1}{2}\left|\mathbf{T}\left(\mathbf{v}^{(n)}\right)\right|^{2}+\eta_{-} \int_{\Omega_{T}} \frac{\partial}{\partial t} \frac{1}{2}\left|\mathbf{T}\left(\mathbf{v}^{(n)}\right)\right|^{2} \\
= & \int_{\Omega_{T}}\left(\eta\left(\phi^{(n)}\right)-\eta_{-}\right) \frac{\partial}{\partial t} \frac{1}{2}\left|\mathbf{T}\left(\mathbf{v}^{(n)}\right)\right|^{2}+\left[\eta_{-} \int_{\Omega} \frac{1}{2}\left|\mathbf{T}\left(\mathbf{v}^{(n)}\right)\right|^{2}\right]_{0}^{T} .
\end{aligned}
$$

Thus, we get

$$
\begin{aligned}
{\left[\eta_{-}\right.} & \left.\int_{\Omega} \frac{1}{2}\left|\mathbf{T}\left(\mathbf{v}^{(n)}\right)\right|^{2}\right]_{0}^{T}+\int_{\Omega_{T}}\left(\mathbf{v}_{t}^{(n)}\right)^{2}+\sigma_{1} \int_{\Omega_{T}}\left|\nabla \mathbf{v}_{t}^{(n)}\right|^{2} \\
= & -\int_{\Omega_{T}}\left(\eta\left(\phi^{(n)}\right)-\eta_{-}\right) \mathbf{T}\left(\mathbf{v}^{(n)}\right): \mathbf{T}\left(\mathbf{v}_{t}^{(n)}\right)+\int_{\Omega_{T}} \mu^{(n)} \nabla \phi^{(n)} \cdot \mathbf{v}_{t}^{(n)} \\
& -\int_{\Omega_{T}}\left(\mathbf{v}^{(n)} \cdot \nabla\right) \mathbf{v}^{(n)} \cdot \mathbf{v}_{t}^{(n)}-\int_{\Omega_{T}}\left\{\frac{\left(\rho_{1}^{(n)}\right)^{+}}{1+\sigma\left(\rho_{1}^{(n)}\right)^{+}} \nabla\left[P_{n}\left(V^{(n)}+M_{L}^{\prime}\left(\rho_{1}^{(n)}\right)\right)\right]\right. \\
& \left.+\frac{\left(\rho_{2}^{(n)}\right)^{+}}{1+\sigma\left(\rho_{2}^{(n)}\right)^{+}} \nabla\left[P_{n}\left(-V^{(n)}+M_{L}^{\prime}\left(\rho_{2}^{(n)}\right)\right)\right]\right\} \cdot \mathbf{v}_{t}^{(n)} .
\end{aligned}
$$

Estimating the r.h.s. using the bounds (B.7) together with Gronwall's inequality and some absorptions, we get

$$
\mathbf{v}_{t}^{(n)} \in L^{2}\left(I ; H^{1}(\Omega)\right) \text { uniformly in } n \in \mathbb{N},
$$

but depending on $\sigma, \sigma_{1}, \sigma_{3}, \Lambda_{1}, \Lambda_{2}, L$.

For the $\rho_{0}$ equation let $\psi_{1} \in L^{2}\left(I ; H^{1}(\Omega)\right)$ and test by $P_{n} \psi_{1} \in L^{2}\left(I ; \mathcal{W}_{n}\right)$ in (B.2b). We have pointwise a.e. in time (here suppressing the $n$-index)

$$
\begin{aligned}
\left|\left\langle\rho_{0, t}, \psi_{1}\right\rangle\right|= & \left|\left\langle\rho_{0, t}, P_{n} \psi_{1}\right\rangle\right| \leq\left|\int_{\Omega} K(\phi) \nabla \rho_{0} \cdot \nabla P_{n} \psi_{1}\right|+\left|\int_{\Omega} \rho_{0} \mathbf{v} \cdot \nabla P_{n} \psi_{1}\right| \\
& +\left|\int_{\Omega} K_{0}(\phi)\left(\rho_{0}^{+}\right)^{\alpha} P_{n} \psi_{1}\right|+\left|\int_{\Omega} K_{1}(\phi) \bar{M}_{\Lambda_{1}}\left(\rho_{1}^{+}\right) \bar{M}_{\Lambda_{1}}\left(\rho_{2}^{+}\right) P_{n} \psi_{1}\right| \\
\leq & C\left(\left\|\nabla \rho_{0}\right\|_{L^{2}(\Omega)}+\left\|\rho_{0}\right\|_{L^{3}(\Omega)}\|\mathbf{v}\|_{L^{6}(\Omega)}\right)\left\|\nabla \psi_{1}\right\|_{L^{2}(\Omega)}
\end{aligned}
$$

Copyright (C) by SIAM. Unauthorized reproduction of this article is prohibited. 


$$
+C\left(\left\|\rho_{0}\right\|_{L^{2}(\Omega)}^{\alpha}+\Lambda_{1}^{2}\right)\left\|\psi_{1}\right\|_{L^{2}(\Omega)} .
$$

Here, we used that the $P_{n}$ are uniformly bounded as operators in $\mathcal{L}(\mathcal{W} ; \mathcal{W})$. By Lemma C.1, Sobolev's embedding theorem, and the a priori estimates (B.7) and (B.4), we can further estimate

$$
\begin{aligned}
\left|\int_{0}^{T}\left\langle\rho_{0, t}, \psi_{1}\right\rangle\right| \leq & C\left(\left\|\nabla \rho_{0}\right\|_{L^{2}\left(\Omega_{T}\right)}+\left\|\rho_{0}\right\|_{L^{3}\left(\Omega_{T}\right)}\|\mathbf{v}\|_{L^{6}\left(\Omega_{T}\right)}\right)\left\|\nabla \psi_{1}\right\|_{L^{2}\left(\Omega_{T}\right)} \\
& +C\left(\left\|\rho_{0}\right\|_{L^{2}\left(\Omega_{T}\right)}^{\alpha}+\Lambda_{1}^{2}\right)\left\|\psi_{1}\right\|_{L^{2}\left(\Omega_{T}\right)} .
\end{aligned}
$$

This yields

$$
\rho_{0, t}^{(n)} \in L^{2}\left(I ;\left(H^{1}(\Omega)\right)^{\prime}\right) \text { uniformly in } n \in \mathbb{N},
$$

but depending on $\sigma, \sigma_{1}, \sigma_{3}, \Lambda_{1}, \Lambda_{2}, L$.

In a similar fashion, we may prove

$$
\begin{aligned}
& \rho_{i, t}^{(n)} \in L^{2}\left(I ;\left(H^{1}(\Omega)\right)^{\prime}\right) \text { for } i \in\{1,2\} \text { uniformly in } n \in \mathbb{N}, \\
& \phi_{t}^{(n)} \in L^{2}\left(I ;\left(H^{1}(\Omega)\right)^{\prime}\right) \text { uniformly in } n \in \mathbb{N},
\end{aligned}
$$

but all dependent on $\sigma, \sigma_{3}, \Lambda_{1}, \Lambda_{2}, L$.

Taking the bounds in (B.8), (B.9)-(B.11), (B.7), and (B.4) and using Rellich's imbedding theorem for $\mathbf{v}$, a compactness result of Simon (cf. [27, Cor. 4, p. 85]) for $\phi$, and the Aubin-Lions lemma for all the remaining quantities, we get existence of an appropriate subsequence that is again denoted by the index $n$ such that for any $r<1, \beta<\frac{5}{6}$

$$
\begin{aligned}
& \mathbf{v}^{(n)} \rightarrow \mathbf{v} \text { weakly in } H^{1}\left(\Omega_{T}\right)^{3}, \\
& \text { weakly * in } L^{\infty}\left(I ; H^{1}(\Omega)^{3}\right) \text {, } \\
& \text { and strongly in } H^{r}\left(\Omega_{T}\right)^{3} \text {, } \\
& \rho_{i}^{(n)} \rightarrow \rho_{i} \text { weakly in } H^{1}\left(I ;\left(H^{1}(\Omega)\right)^{\prime}\right) \cap L^{2}\left(I ; H^{1}(\Omega)\right) \text {, } \\
& \text { weakly * in } L^{\infty}\left(I ; L^{2}(\Omega)\right) \text {, } \\
& \text { and strongly in } L^{2}\left(I ; H^{r}(\Omega)\right) \text { for } i \in\{0,1,2\} \text {, } \\
& \phi^{(n)} \rightarrow \phi \text { weakly in } H^{1}\left(I ;\left(H^{1}(\Omega)\right)^{\prime}\right), \\
& \text { weakly * in } L^{\infty}\left(I ; W^{1, s}(\Omega)\right) \text {, } \\
& \text { and strongly in } C^{0}\left(\bar{I} ; C^{0, \beta}(\bar{\Omega})\right) \text {, } \\
& \phi^{(n)} \rightarrow \phi \text { weakly in } H^{1}\left(I ; L^{2}(\partial \Omega)\right) \text {, } \\
& \text { and strongly in } L^{2}(\partial \Omega \times I) \text {, } \\
& \phi^{(n)}(t, x) \rightarrow \phi(t, x) \text { for a.e. } t \in I, x \in \Omega \text {, } \\
& \nabla \mu^{(n)} \rightarrow \nabla \mu \text { weakly in } L^{2}\left(\Omega_{T}\right), \\
& V^{(n)} \stackrel{*}{\rightarrow} V \text { weakly * in } L^{\infty}\left(I ; H^{1}\left(\Omega^{*}\right)\right) \text {. }
\end{aligned}
$$

As in [11, p. 278], one can show

$$
V^{(n)} \rightarrow V \text { strongly in } L^{2}\left(I ; H^{1}\left(\Omega^{*}\right)\right) .
$$

Copyright (c) by SIAM. Unauthorized reproduction of this article is prohibited. 
Limit $n \rightarrow \infty$. With convergences (B.12) and (B.13) at hand, we now pass with $n \rightarrow \infty$ in (B.2), (B.3), and (B.6). We start with the latter ones. Since the r.h.s. of (B.3) and (B.6) are uniformly bounded in $n \in \mathbb{N}$, we can use the lower semicontinuity of the norm with respect to weak/weak ${ }^{*}$ convergence for all of the terms on the l.h.s. of (B.3) and most of the terms on the l.h.s. of (B.6). The three remaining terms in (B.6) are as follows. The convergence

$$
\int_{\Omega} M_{L}\left(\rho_{i}\right) \leq \liminf _{n \rightarrow \infty} \int_{\Omega} M_{L}\left(\rho_{i}^{(n)}\right)
$$

is Fatou's lemma. Since

$$
\int_{\Omega_{T}}\left|P_{n}\left((-1)^{i+1} V^{(n)}+M_{L}^{\prime}\left(\rho_{i}^{(n)}\right)\right)\right|^{2}=: \int_{\Omega_{T}}\left(R_{1}^{(n)}\right)^{2}
$$

and

$$
\int_{\Omega_{T}} K\left(\phi^{(n)}\right) \frac{1}{M_{L}^{\prime \prime}\left(\rho_{i}^{(n)}\right)}\left|\nabla\left[P_{n}\left((-1)^{i+1} V^{(n)}+M_{L}^{\prime}\left(\rho_{i}^{(n)}\right)\right)\right]\right|^{2}=: \int_{\Omega_{T}}\left(R_{2}^{(n)}\right)^{2}
$$

are uniformly bounded in $n \in \mathbb{N}$, we get for $i \in\{1,2\}$

$$
\int_{\Omega_{T}} R_{i}^{2} \leq \liminf _{n \rightarrow \infty} \int_{\Omega_{T}}\left(R_{i}^{(n)}\right)^{2}
$$

also by lower semicontinuity of the norm. Since $P_{n}$ is uniformly bounded in $\mathcal{L}(\mathcal{W} ; \mathcal{W})$ and $P_{n} v \rightarrow v$ strongly in $H^{1}(\Omega)$ for all $v \in H^{1}(\Omega)$, we get with the help of (B.13) the strong convergence $P_{n} V_{n} \rightarrow V$ in $H^{1}(\Omega) . M_{L}^{\prime}(\rho)$ has linear growth in $\rho$. Thus, we get, using Lebesgue's theorem and the strong convergence of $\rho_{i}$ for $i \in\{1,2\}$,

$$
\int_{\Omega_{T}} P_{n}\left(M_{L}^{\prime}\left(\rho_{i}^{(n)}\right)\right) \phi=\int_{\Omega_{T}} M_{L}^{\prime}\left(\rho_{i}^{(n)}\right) P_{n} \phi \rightarrow \int_{\Omega_{T}} M_{L}^{\prime}\left(\rho_{i}\right) \phi
$$

for all step functions $\phi$ in time with values in $L^{2}(\Omega)$. Since step functions are dense in $L^{2}\left(\Omega_{T}\right)$, we have

$$
\mathrm{w}-\lim _{n \rightarrow \infty} P_{n}\left(M_{L}^{\prime}\left(\rho_{i}^{(n)}\right)\right)=M_{L}^{\prime}\left(\rho_{i}\right) .
$$

This yields $R_{1}=\left((-1)^{i+1} V+M_{L}^{\prime}\left(\rho_{i}\right)\right)$. Since $K(\phi) \frac{1}{M_{L}^{\prime \prime}(\rho)}$ is uniformly bounded and strictly positive, we get with Lebesgue's theorem and the strong convergences of $\rho_{i}^{(n)}$ and $\phi^{(n)}$ that

$$
R_{2}=\left(K(\phi) \frac{1}{M_{L}^{\prime \prime}(\rho)}\right)^{1 / 2} \nabla\left(V+M_{L}^{\prime}(\rho)\right) .
$$

Thus, from (B.6) we get

$$
\begin{aligned}
\underset{t \in(0, T)}{\operatorname{ess~sup}}[ & \int_{\Omega}\left\{\frac{1}{2}|\mathbf{v}|^{2}+\frac{\sigma_{1}}{2}|\nabla \mathbf{v}|^{2}+M_{L}\left(\rho_{1}\right)+M_{L}\left(\rho_{2}\right)+\frac{1}{s}|\nabla \phi|^{s}\right. \\
& \left.+W(\phi)\}+\int_{\partial \Omega} \gamma_{f s}(\phi)+\int_{\Omega^{*}} \frac{1}{2} \bar{\varepsilon}[\phi]|\nabla V|^{2}\right](t) \\
+ & \int_{\Omega_{T}}\left[\eta(\phi)|\mathbf{T}(\mathbf{v})|^{2}+\sigma_{3}\left|V+M_{L}^{\prime}\left(\rho_{1}\right)\right|^{2}+\sigma_{3}\left|-V+M_{L}^{\prime}\left(\rho_{2}\right)\right|^{2}\right.
\end{aligned}
$$

Copyright (c) by SIAM. Unauthorized reproduction of this article is prohibited. 


$$
\begin{aligned}
& \quad+K(\phi) \frac{1}{M_{L}^{\prime \prime}\left(\rho_{1}\right)}\left|\nabla\left[V+M_{L}^{\prime}\left(\rho_{1}\right)\right]\right|^{2}+K(\phi) \frac{1}{M_{L}^{\prime \prime}\left(\rho_{2}\right)}\left|\nabla\left[-V+M_{L}^{\prime}\left(\rho_{2}\right)\right]\right|^{2} \\
& \left.+M(\phi)|\nabla \mu|^{2}\right]+\int_{\partial \Omega_{T}} \alpha\left|\phi_{t}\right|^{2} \\
& \leq C\left(\mathbf{v}_{0}, \rho_{0,0}, \rho_{1,0}, \rho_{2,0}, \phi_{0}, V_{0}\right)-\int_{\Omega_{T}^{*}} \bar{\varepsilon}[\phi] \nabla V \nabla \bar{V}_{t}+\left[\int_{\Omega^{*}} \bar{\varepsilon}[\phi] \nabla V \nabla \bar{V}\right]_{0}^{T} \\
& +\int_{\Omega_{T}}\left(\rho_{1}-\rho_{2}\right) \bar{V}_{t}-\left[\int_{\Omega}\left(\rho_{1}-\rho_{2}\right) \bar{V}\right]_{0}^{T} \\
& -\sigma_{2} \int_{\Omega_{T}} \nabla \rho_{1} \cdot \nabla\left(V+M_{L}^{\prime}\left(\rho_{1}\right)\right)-\sigma_{2} \int_{\Omega_{T}} \nabla \rho_{2} \cdot \nabla\left(-V+M_{L}^{\prime}\left(\rho_{2}\right)\right) \\
& \quad-\int_{\Omega_{T}} K_{1}(\phi) \bar{M}_{\Lambda_{2}}\left(\rho_{1}^{+}\right) \bar{M}_{\Lambda_{2}}\left(\rho_{2}^{+}\right)\left(M_{L}^{\prime}\left(\rho_{1}\right)+M_{L}^{\prime}\left(\rho_{2}\right)\right) \\
& +\int_{\Omega_{T}} K_{0}(\phi)\left(\rho_{0}^{+}\right)^{\alpha}\left(M_{L}^{\prime}\left(\rho_{1}\right)+M_{L}^{\prime}\left(\rho_{2}\right)\right) \\
& \leq \bar{C}\left(\mathbf{v}_{0}, \rho_{0,0}, \rho_{1,0}, \rho_{2,0}, \phi_{0}, V_{0}, \bar{V}\right) .
\end{aligned}
$$

By compensated compactness (see, e.g., [30]), we obtain easily

$$
\mathbf{v}^{(n)} \cdot \nabla \rho^{(n)} \rightarrow \mathbf{v} \cdot \nabla \rho \quad \text { and } \quad \mathbf{v}^{(n)} \cdot \nabla \phi^{(n)} \rightarrow \mathbf{v} \cdot \nabla \phi \quad \text { weakly in } L^{p}\left(\Omega_{T}\right)
$$

for some $p>1$, since $\nabla \cdot \mathbf{v}^{(n)}=0$ and $\nabla \rho^{(n)}, \nabla \phi^{(n)}$ are $L^{2}$-integrable gradient fields. Since $\phi^{(n)} \rightarrow \phi$ a.e., we have also for a subsequence

$$
\varepsilon\left(\phi^{(n)}\right) \rightarrow \varepsilon(\phi), \varepsilon^{\prime}\left(\phi^{(n)}\right) \rightarrow \varepsilon^{\prime}(\phi), \text { and } \eta\left(\phi^{(n)}\right) \rightarrow \eta(\phi) \text { a.e. }
$$

We recall the boundedness of $\varepsilon, \varepsilon^{\prime}$, and $\eta$ and obtain easily the strong convergence in $L^{p}\left(\Omega_{T}\right)$ for arbitrary $p<+\infty$. These convergences and the pseudomonotonicity of $-\Delta_{s}$ (see Lemma C.6) allow us to pass to the limit in the Galerkin discretization. In (B.2a) the convergence

$$
\int_{\Omega_{T}} \rho_{0}^{(n)} \nabla V^{(n)} \cdot \mathbf{w} \rightarrow \int_{\Omega_{T}} \rho_{0} \nabla V \cdot \mathbf{w}
$$

follows from the strong convergence of $\rho$ in $L^{2}\left(\Omega_{T}\right)$; the convergence

$$
\int_{\Omega_{T}} \mu^{(n)} \nabla \phi^{(n)} \cdot \mathbf{w} \rightarrow \int_{\Omega_{T}} \mu \nabla \phi \cdot \mathbf{w}
$$

is derived after an integration by parts from

$$
\int_{\Omega_{T}} \phi^{(n)} \nabla \mu^{(n)} \cdot \mathbf{w} \rightarrow \int_{\Omega_{T}} \phi \nabla \mu \cdot \mathbf{w}
$$

For the limit process in (B.2e) note that the equation is of the form

$$
\left\langle-\Delta_{s} \phi^{(n)}, \psi_{4}\right\rangle_{X^{\prime} \times X}=\int_{\Omega_{T}} b^{(n)} \psi_{4} \quad \text { for all } \psi_{4} \in X
$$

with $b^{(n)}:=\mu^{(n)}+\frac{1}{2} \varepsilon^{\prime}\left(\phi^{(n)}\right)\left|\nabla V^{(n)}\right|^{2}-W^{\prime}\left(\phi^{(n)}\right), X=L^{s}\left(I ; W^{1, s}(\Omega)\right) \cap H^{1}\left(I ; L^{2}(\partial \Omega)\right)$. From the convergences (B.12) and (B.13), we get $b^{(n)} \rightarrow b=-W^{\prime}(\phi)+\mu+\frac{1}{2} \varepsilon^{\prime}(\phi)|\nabla V|^{2}$ 
weakly in $L^{2}\left(I ; L^{1}(\Omega)\right)$, which in particular implies $-\Delta_{s} \phi^{(n)} \stackrel{*}{\rightarrow} b$ weakly in $X^{\prime}$. By the weak convergence of $b^{(n)}$ and the strong convergence of $\phi^{(n)}$, we get

$$
\lim _{n \rightarrow \infty}\left\langle-\Delta_{s} \phi^{(n)}, \phi^{(n)}\right\rangle_{X^{\prime} \times X}=\lim _{n \rightarrow \infty} \int_{\Omega_{T}} b^{(n)} \phi^{(n)}=\int_{\Omega_{T}} b \phi .
$$

By the pseudomonotonicity of $-\Delta_{s}$ (see Lemma C.6), identity (B.15), and [26, Prop. 2.3 , p. 41], the limit of (B.2e) can be identified with (B.1e).

\section{Appendix C. Auxiliary results from analysis.}

Lemma C.1 (see $\left[9\right.$, p. 8]). Let $\Omega \subset \mathbb{R}^{3}$ with piecewise smooth boundary. Then there exists a constant depending only on $p, m$, and the structure of $\partial \Omega$ such that for every $u \in L^{\infty}\left(I ; L^{m}(\Omega)\right) \cap L^{p}\left(I ; W^{1, p}(\Omega)\right)$

$$
\|u\|_{L^{q}\left(I ; L^{q}(\Omega)\right)} \leq C\left(\|u\|_{L^{\infty}\left(I ; L^{m}(\Omega)\right)}+\|\nabla u\|_{L^{p}\left(\Omega_{T}\right)}\right)
$$

with $q=p \frac{3+m}{3}$.

Lemma C.2 (see [9, p. 10]). Let $\Omega \subset \mathbb{R}^{3}$ with piecewise smooth boundary. Then there exists a positive constant depending only on $p$ and the structure of $\partial \Omega$ such that for every $u \in L^{\infty}\left(I ; L^{p}(\Omega)\right) \cap L^{p}\left(I ; W^{1, p}(\Omega)\right)$

$$
\|u\|_{L^{r}\left(I ; L^{q}(\Omega)\right)} \leq C\left(\|u\|_{L^{\infty}\left(I ; L^{p}(\Omega)\right)}+\|\nabla u\|_{L^{p}\left(\Omega_{T}\right)}\right),
$$

where $q, r \geq 1$ are linked by $\frac{1}{r}+\frac{3}{p q}=\frac{3}{p^{2}}$ and their admissible range is

$$
\begin{array}{ll}
q \in\left[p, \frac{3 p}{3-p}\right], \quad r \in[p, \infty] & \text { if } 1 \leq p<3, \\
q \in[p, \infty), \quad r \in\left(\frac{p^{2}}{3}, \infty\right] & \text { if } 1<3 \leq p .
\end{array}
$$

Lemma C.3 ( $L^{p}$ interpolation in time-space). Let $u \in L^{\infty}\left(I ; L^{2}(\Omega)\right) \cap L^{r}\left(I ; L^{p}(\Omega)\right)$. Then for $1 \leq r<\infty, 1 \leq p \leq \infty$, we have

$$
u \in L^{q}\left(\Omega_{T}\right) \text { with } q=2\left(1-\frac{r}{p}+\frac{r}{2}\right) .
$$

Lemma C.4 (Gagliardo-Nirenberg). Let $1 \leq r \leq \infty, p \geq 1, q \in[1, p), m \in \mathbb{N}_{+}$ such that $\frac{1}{r}-\frac{m}{n}<\frac{1}{p}$. If $\Omega \subset \mathbb{R}^{n}$ is bounded with piecewise smooth boundary, then positive constants $c_{1}$ and $c_{2}$ depending only on $\Omega, r, p, m$, and $q$ exist such that for any $u \in L^{q}(\Omega)$ satisfying $D^{m} u \in L^{r}(\Omega)$ the following inequality holds:

$$
\|u\|_{L^{p}(\Omega)} \leq c_{1}\left\|D^{m} u\right\|_{L^{r}(\Omega)}^{a}\|u\|_{L^{q}(\Omega)}^{1-a}+c_{2}\|u\|_{L^{q}(\Omega)},
$$

where $a=\frac{\frac{1}{q}-\frac{1}{p}}{\frac{1}{q}+\frac{m}{n}-\frac{1}{r}}$.

Lemma C.5 (see Di Fazio [10, Theorem 2.1]). Let $\Omega \subset \mathbb{R}^{n}, n \geq 3$, with boundary of class $C^{1,1}$. Consider the elliptic equation

$$
-\sum_{i, j=1}^{n} \partial_{x_{j}}\left(a_{i j} \partial_{x_{i}} u\right)=\nabla \cdot \mathbf{f} \quad \text { in } \Omega .
$$

Assume

- $a_{i j} \in V M O \cap L^{\infty}(\Omega)$ for $i, j=1, \ldots, n$,

Copyright (c) by SIAM. Unauthorized reproduction of this article is prohibited. 
- $a_{i j}=a_{j i}$ and there exists $\nu>0$ such that $\nu^{-1}|\xi|^{2} \leq \sum_{i, j=1}^{n} a_{i j} \xi_{i} \xi_{j} \leq \nu|\xi|^{2}$ for all $\xi \in \mathbb{R}^{n}$ and for a.e. $x \in \Omega$,

- there exists some $p \in(1,+\infty)$ such that $\mathbf{f} \in L^{p}(\Omega)^{n}$;

then the homogeneous Dirichlet problem has a (unique) weak solution $u \in W_{0}^{1, p}(\Omega)$ and, moreover, there exists a constant $c=c\left(n, p, \nu, a_{i j}, \partial \Omega\right)$ such that

$$
\|\nabla u\|_{L^{p}(\Omega)} \leq c\|\mathbf{f}\|_{L^{p}(\Omega)} .
$$

Lemma C.6 (pseudomonotonicity). The operator $-\Delta_{s}: X \rightarrow X^{\prime}$ with $X:=$ $L^{s}\left(I ; W^{1, s}(\Omega)\right) \cap H^{1}\left(I ; L^{2}(\partial \Omega)\right)$ defined by

$$
\left\langle-\Delta_{s} \phi, \psi\right\rangle_{X^{\prime} \times X}:=\int_{\Omega_{T}}|\nabla \phi|^{s-2} \nabla \phi \cdot \nabla \psi+\int_{\partial \Omega_{T}}\left(\alpha \phi_{t}+\gamma_{f s}^{\prime}(\phi)\right) \psi
$$

for all $\psi \in X$ is pseudomonotone; i.e.,

$$
\phi_{n} \rightarrow \phi \text { weakly in } X \quad \text { and } \quad \limsup _{n \rightarrow \infty}\left\langle-\Delta_{s} \phi_{n}, \phi_{n}-\phi\right\rangle_{X^{\prime} \times X} \leq 0
$$

imply

$$
\left\langle-\Delta_{s} \phi, \phi-\psi\right\rangle_{X^{\prime} \times X} \leq \limsup _{n \rightarrow \infty}\left\langle-\Delta_{s} \phi_{n}, \phi_{n}-\psi\right\rangle_{X^{\prime} \times X} \text { for all } \psi \in X .
$$

Proof. This result can be proved by standard methods in the theory of monotone operators; see [26].

Acknowledgment. Parts of this paper had been written while G. Grün and S. Jörres visited Universidad Autonoma de Madrid and while M. A. Fontelos visited Erlangen University. The warm hospitality of the host institutions is gratefully acknowledged by the authors.

\section{REFERENCES}

[1] H. ABELS, On a diffuse interface model for two-phase flows of viscous, incompressible fluids with matched densities, Arch. Ration. Mech. Anal., 194 (2009), pp. 463-506.

[2] H. ABELs, Existence of weak solutions for a diffuse interface model for viscous, incompressible fluids with general densities, Comm. Math. Phys., 289 (2009), pp. 45-73.

[3] R. A. Adams and J. J. Fournier, Sobolev Spaces, 2nd ed., Academic Press, Amsterdam, 2003.

[4] B. Berge And J. Peseux, Variable focal lens controlled by an external voltage: An application of electrowetting, Eur. Phys. J. E Soft Matter Biol. Phys., 3 (2000), pp. 159-163.

[5] T. D. Blake, A. Clarke, and E. H. Stattersfield, An investigation of electrostatic assist in dynamic wetting, Langmuir, 16 (2000), pp. 2928-2935.

[6] A. Castellanos, ED., Electrohydrodynamics, CISM Courses and Lectures 380, Springer, Wien, 1998.

[7] C. G. Cooney, C. Y. Chen, M. Emerling, A. Nadim, and J. D. Sterling, Electrowetting droplet microfluidics on a single planar surface, Microfluidics and Nanofluidics, 2 (2006), pp. 435-446.

[8] R. Dal Passo, H. Garcke, and G. Grün, On a fourth-order degenerate parabolic equation: Global entropy estimates, existence, and qualitative behavior of solutions, SIAM J. Math. Anal., 29 (1998), pp. 321-342.

[9] E. DiBenedetto, Degenerate Parabolic Equations, Springer, New York, 1993.

[10] G. Di FAZIO, $L^{p}$ estimates for divergence form elliptic equations with discontinuous coefficients, Boll. Un. Mat. Ital. A (7), 10 (1996), pp. 409-420.

[11] C. Eck, M. Fontelos, G. Grün, F. Klingbeil, And O. Vantzos, On a phase-field model for electrowetting, Interfaces Free Bound., 11 (2009), pp. 259-290.

[12] A. Jüngel, A nonlinear drift-diffusion system with electric convection arising in electrophoretic and semiconductor modeling, Math. Nachr., 185 (1997), pp. 85-110.

Copyright $@$ by SIAM. Unauthorized reproduction of this article is prohibited. 
[13] D. Kay, V. Styles, And R. Welford, Finite element approximation of a Cahn-HilliardNavier-Stokes system, Interfaces Free Bound., 10 (2008), pp. 15-43.

[14] S. Kuiper and B. H. W. Hendricks, Variable-focus liquid lens for miniature cameras, Appl. Phys. Lett., 85 (2004), pp. 1128-1130.

[15] J. S. Lowengrub And L. Truskinovsky, Quasi-incompressible Cahn-Hilliard fluids and topological transitions, R. Soc. Lond. Proc. Ser. A Math. Phys. Eng. Sci., 454 (1998), pp. $2617-$ 2654.

[16] H. W. Lu, K. Glasner, A. L. Bertozzi, and C. J. Kim, A diffuse interface model for electrowetting drops in a Hele-Shaw cell, J. Fluid Mech., 590 (2007), pp. 411-435.

[17] H. Morgan and N. G. Green, AC Electrokinetics: Colloids and Nanoparticles, Research Studies Press, Hertfordshire, England, 2003.

[18] F. Mugele and J. C. Baret, Electrowetting: From basics to applications, J. Phys. Condens. Matter, 17 (2005), pp. R705-R774.

[19] L. Onsager, Reciprocal relations in irreversible processes I., Phys. Rev., 37 (1931), pp. 405426.

[20] M. W. J. Prins, W. J. J. Welters, AND J. W. Weekamp, Fluid control in multichannel structures by electrocapillary pressure, Science, 291 (2001), pp. 277-280.

[21] T. QIAn, X. P. WANG, AND P. Sheng, A variational approach to moving contact line hydrodynamics, J. Fluid Mech., 564 (2006), pp. 333-360.

[22] C. Quillet and B. Berge, Electrowetting: A recent outbreak, Current Opinion in Colloid \& Interface Sci., 6 (2001), pp. 34-39.

[23] D. A. SAville, Electrohydrodynamics: The Taylor-Melcher leaky dielectric model, Annu. Rev. Fluid Mech., 29 (1997), pp. 27-64.

[24] M. Schmuck, Analysis of the Navier-Stokes-Nernst-Planck-Poisson system, Math. Models Methods Appl. Sci., 19 (2009), pp. 993-1015.

[25] M. Schmuck, Modeling, Analysis, and Numerics in Electrohydrodynamics, Dissertation, Universität Tübingen, 2008.

[26] R. E. Showalter, Monotone Operators in Banach Space and Nonlinear Partial Differential Equations, AMS, Providence, RI, 1997.

[27] J. Simon, Compact sets in the space $L^{p}(0, T ; B)$, Ann. Mat. Pura Appl. (4), 146 (1986), pp. 6596.

[28] T. M. Squires and M. Z. BAzant, Induced-charge electro-osmosis, J. Fluid. Mech., 509 (2004), pp. $217-252$.

[29] H. A. Stone, A. D. Stroock, And A. Ajdari, Engineering flows in small devices: Microfluidics toward a lab-on-a-chip, Annu. Rev. Fluid Mech., 36 (2004), pp. 381-411.

[30] M. Struwe, Variational Methods, Springer, Berlin, 1990.

[31] M. Vallet, M. Vallade, AND B. Berge, Limiting phenomena for the spreading of water on polymer films by electrowetting, Eur. Phys. J. B Condens. Matter Phys., 11 (1999), pp. 583-591.

[32] S. W. Walker, A. Bonito, And R. Nochetto, Mixed finite element method for electrowetting on dielectric with contact line pinning, Interfaces Free Bound., 12 (2010), pp. 85-119.

[33] S. W. Walker and B. Shapiro, Modeling the fluid dynamics of electrowetting on dielectric (EWOD), J. Microelectromechanical Systems, 15 (2006), pp. 986-1000.

Copyright (c) by SIAM. Unauthorized reproduction of this article is prohibited. 